\title{
SUMMARY OF THE
}

\section{"DATABASE OF NGO ACTIVITIES"}

\section{AGENCY COORDINATING BODY FOR AFGHAN RELIEF \\ 2 Rehman Baba Road University. Town \\ Peshawar}

Tel: (0521) $44392 / 40839$

Fax: (0521) 840471 

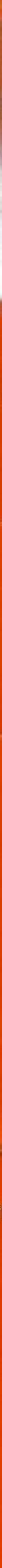

Printed By: IRC Rrinting Pŗess 


\section{CONTENTS}

A. Introduction $\ldots \ldots \ldots \ldots \ldots \ldots \ldots \ldots \ldots \ldots \ldots \ldots \ldots \ldots \ldots \ldots$

B. Number of NGOs in different sectors in different Provinces . . . . . 3

C. NGOs' sector of work in each District by District during $1995 \ldots$. . 4

D. Number of NGOs in different Provinces during 1995 (Graph) . . 19

E. NGOs working in each District during $1995 \ldots \ldots \ldots 20$

F. Number of NGOs in different sectors during 1995 (Graph) . . . . 35

G. NGOs sector of work by NGO during $1995 \ldots \ldots \ldots$. . . . . 36

H. Operation sites of NGOs during $1995 \ldots \ldots \ldots \ldots \ldots \ldots \ldots$

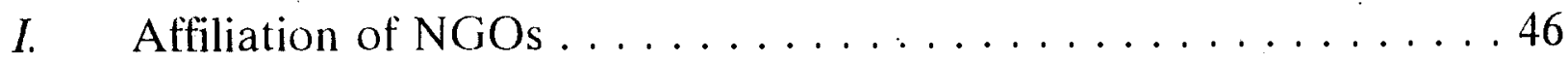

J. NGOs' funding sources during $1995 \ldots \ldots \ldots \ldots$. . . . . . . 51

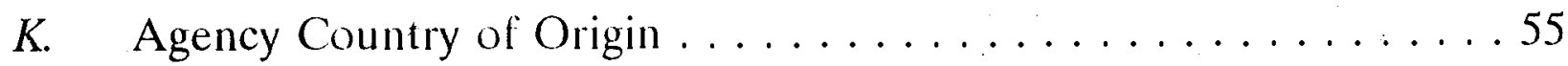

L. Location codes . . . . . . . . . . . . . . . . . . . 61

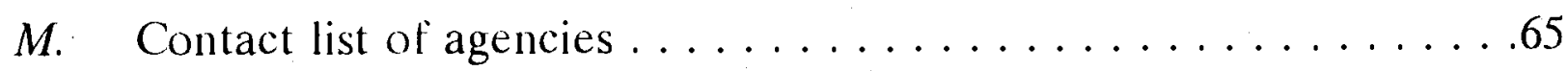

N. Amendments to the 8 th edition of the Database . . . . . . . . 70 


\section{INTRODUCTION}

The purpose of Summary of the Database of NGO Activities is to provide users with a quick reference to know who is doing what and where. It will also help users identify the gaps and thus facilitate their future planning.

The Summary also contains extra information on agencies such as agency country of origin and affiliation. Information on donor agencies has also been included in the Summary.

Finally, an amendment page for errors in the 8 th edition of the Database has also been added. 
NUMBER OF NGOS IN DIFFERENT SECTORS IN DIFFERENT PROVINCES DURING 1995

\begin{tabular}{|c|c|c|c|c|c|c|c|c|c|c|c|}
\hline PROVINCES & $\begin{array}{l}\mathrm{AGR} \\
\mathrm{ICU} \text {. }\end{array}$ & $\begin{array}{l}\text { Con } \\
\text { st: }\end{array}$ & $\begin{array}{l}\text { EDU } \\
\text { CAT }\end{array}$ & EPI & $\begin{array}{l}\text { IIEA } \\
\text { LTH }\end{array}$ & INC: & Mi & RELII & $\begin{array}{l}\text { soc } \\
141\end{array}$ & VET: & $\begin{array}{l}\text { WA } \\
\text { TER }\end{array}$ \\
\hline BADKHSHAN & 5 & 4 & 1 & 0 : & 3 & 0 & 1 & 0 & 1 & 2 & 2 \\
\hline BADGHIS & 0 & 0 & 0 & 0 & 1 & 0 & 0 & 0 & 0 & 1 & 0 \\
\hline BAGHIAN & 4 & 5 & 1 & 1 & 6 & 5 & 1 & $I$ & 3 & 1 & 5 \\
\hline BALKH & 1 & 6 & 4 & 0 & 8 & 5 & 0 & 1 & 3 & 1 & 4 \\
\hline BAMYAN. & 1 & 2 & 4 & 1 & 5 & 3 & 0 & 0 & 1 & 1 & 0 \\
\hline FARAH. & 4 & 1 & 0 & 2 & 3 & 1 & 0 & 0 & 0 & 1 & 2 \\
\hline FARYAB & 0 & 2 & 1 & 0 & 3 & 1 & 0. & 0. & 8 & 8 & 2 \\
\hline GHAZNI & 10 & 8 & 8 & 3 & 13 & 8 & 3 & 1 & 4 & 5 & 8 \\
\hline GHOR & 2 & 0 & 0 & $I$ & 1 & 0 & 0 & 0 & 0 & 0 & 0 \\
\hline IIELMAND & 6 & 2 & 0 & 1 & 5 & 2 & 3 & 0 & 1 & 2 & 6 \\
\hline IERIT & 5 & 4 & 4 & 1 & 8 & 4 & 3 & 3 & 1 & 2 & 5 \\
\hline$J A W Z J A N$ & 0 & 2 & 1 & 0 & 3 & 3 & 0 & 1 & 2 & 0. & 4 \\
\hline KIBUI & 11 & 13 & 5 & 1 & 23 & 8 & 5 & 11 & 2 & 1 & 9 \\
\hline KANDAHAR & 11 & 17 & 5 & 1 & 6 & 3 & 3 & 0 & 4 & 2 & 32 \\
\hline KIPISA & 2 & 2 & 2 & $I$ & 4 & $I$ & 1 & 0 & 8 & 2 & 2 \\
\hline KUNAR & 11 & 15 & 8 & 1 & 11. & 6 & 0 & 0 & 2 & 3 & 5 \\
\hline KUNDUZ & 2 & 2 & 1 & 0 & 5 & 3 & 0 & $I$ & 1 & 1 & 2 \\
\hline LIGHMAN & 18 & 15 & 8 & 1 & 10 & 4 & 0 & 0 & 0 & 2 & 10 \\
\hline LOGAR & 13 & 6 & 9 & 1 & 9 & 3 & 3 & 8 & 1 & 4 & 5 \\
\hline NANGARHIR & 38 & 40 & 2.5 & 1 & 38 & 22 & 5 & 12 & 4 & 3 & 16 \\
\hline NIMROZ & 1 & 2 & 1 & 0 & 3 & 1 & 1 & 1 & 0 & 1 & 3 \\
\hline ORUZGAN & 3 & 1 & 2 & 1 & 4 & 1 & 3 & 0 & 0 & 2 & 4 \\
\hline PAKTEKA. & 8 & 2 & 3 & 1 & 2 & 0 & 0 & 0 & 0 & 2 & 2 \\
\hline PAKTYA. & 19 & 13 & 10 & 1 & 7 & 5 & 4 & 0 & 4 & 3 & 14 \\
\hline PARWAN & 5 & 4 & 3 & 2 & 9 & 2 & 4 & 0 & 1 & 2 & 4 \\
\hline SAMANGAN & 0 & 0 & 2 & 0 & 2 & 2 & 1 & 0 & 1 & 0 & 2 \\
\hline TAKHAR. & 3 & 2 & 1 & 0 & 3 & 1 & 0 & 0 & 1 & 1 & 2 \\
\hline WIRDAR & 12 & 7 & 8 & 2 & 10 & 6 & 0 & 1 & $I$ & 2 & 4 \\
\hline ZaBul & 2 & 2 & 1 & 1 & 1 & $I$ & 3 & 0 & 1 & 1 & 8 \\
\hline
\end{tabular}


NGOS SECTOR OF WORK IN EACH DISTRICT BY DISTRICT DURING 1995

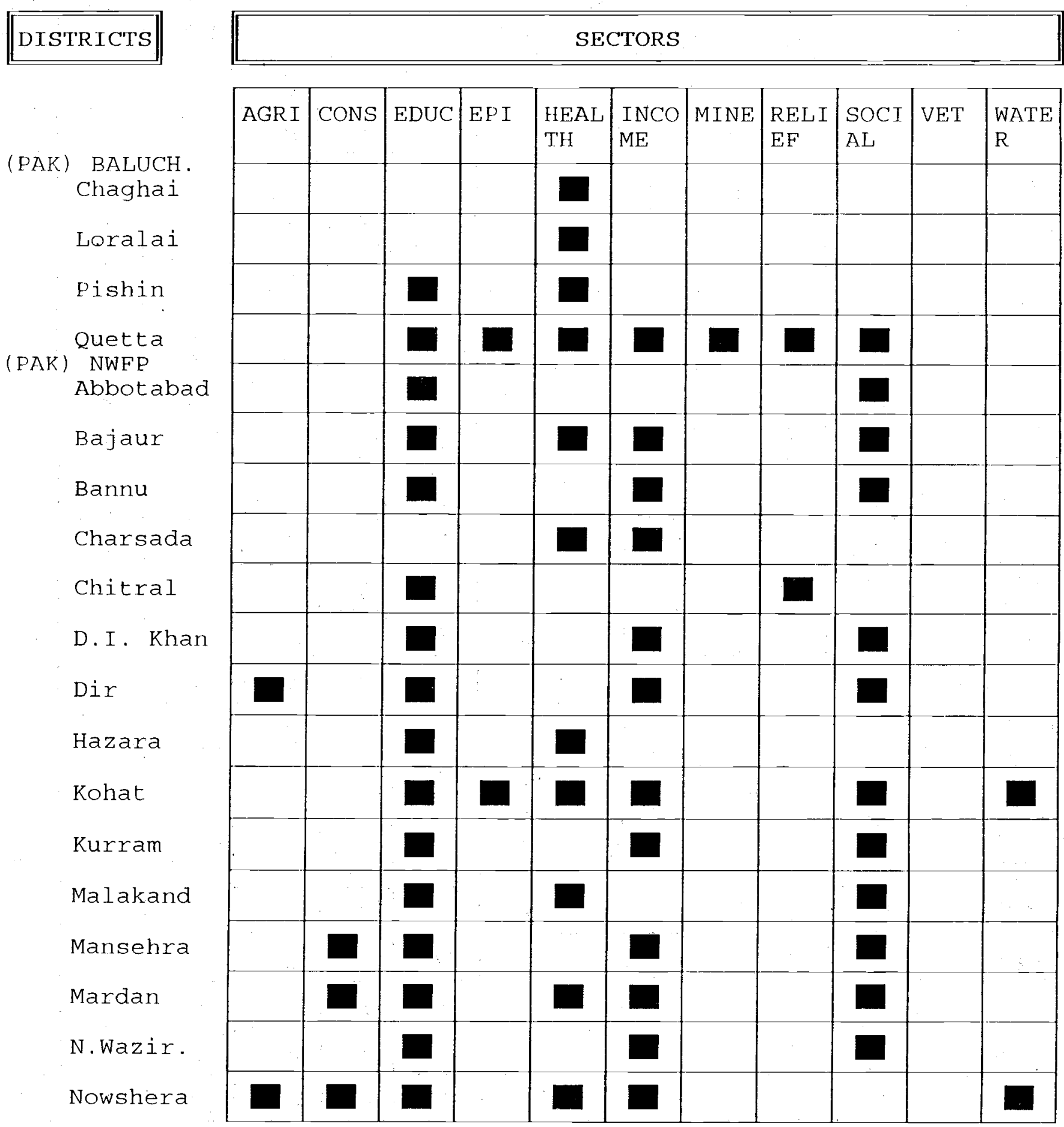




\begin{tabular}{|c|c|c|c|c|c|c|c|c|c|c|c|}
\hline DISTRICTS & \multicolumn{11}{|c|}{ SECTORS } \\
\hline & AGRI & CONS & EDUC & EPI & $\begin{array}{l}\text { HEAL } \\
\text { TH }\end{array}$ & $\begin{array}{l}\text { INCO } \\
\mathrm{ME}\end{array}$ & MINE & $\begin{array}{l}\text { RELI } \\
\text { EF }\end{array}$ & $\begin{array}{l}\text { SOC.L } \\
\text { AL }\end{array}$ & VET & $\begin{array}{l}\text { WAT'E } \\
\mathrm{R}\end{array}$ \\
\hline Orakzai & & & & & & & & & & & \\
\hline Peshawar & & & & & & & & & & & \\
\hline S.Wazir. & & & & & & & & & & & \\
\hline $\begin{array}{c}\text { Swàt } \\
\text { (PAK) PUNJAB }\end{array}$ & & & & & & & & & & & \\
\hline Islamabad & & & & & & & & & & & \\
\hline Mian Wali & & & & & & & & & & & \\
\hline Baharak & & & & & & & & & & & \\
\hline Darwaz & & & & & & & & & & & \\
\hline Eshkashem & & & & . & & & & & & & \\
\hline Faizabad & & & & & & & & & & & \\
\hline iJurm & & & & & & & & & & & \\
\hline Keranomon. & & & & & & & & & & & \\
\hline Keshem & & & & & & & & & & & \\
\hline Khwahan & & & & & & & & & & & \\
\hline Ragh & & & & & & & & & & & \\
\hline Shar Boz: & & & & & & & & 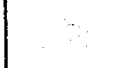 & & & \\
\hline Sheghnan & & & & & & & & & & & \\
\hline Wakhan & & & & & & & & & & & \\
\hline $\begin{array}{l}\text { Zebak } \\
\text { BADGHIS }\end{array}$ & & & & & & & & & & & \\
\hline Ghormach & & & & & & & & & & & \\
\hline Jawand & & & & & & & & & & & \\
\hline Kushk Koh. & & & & & & & & & & & \\
\hline Morghab & & & & & & & & & & & \\
\hline Qades & & & & & & & & & & & \\
\hline Qala Nau & & & & & & & & & & & \\
\hline
\end{tabular}




\begin{tabular}{|c|c|c|c|c|c|c|c|c|c|c|c|}
\hline \multirow[t]{2}{*}{\begin{tabular}{|l|} 
DISTRICTS \\
\end{tabular}} & \multicolumn{11}{|c|}{ SECTORS } \\
\hline & AGRI & CONS & EDUC & EPI & $\begin{array}{l}\text { HEAL } \\
\text { TH }\end{array}$ & $\begin{array}{l}\text { INCO } \\
\text { ME }\end{array}$ & MINE & $\begin{array}{l}\text { RELI } \\
\text { EFF }\end{array}$ & $\begin{array}{l}\text { SOCI } \\
\text { AL }\end{array}$ & VET & $\begin{array}{l}\text { WATE } \\
\text { R }\end{array}$ \\
\hline $\begin{array}{l}\text { BAGHLAN } \\
\text { Anderab }\end{array}$ & $\square$ & $\bar{\square}$ & $\square$ & & $\square$ & & & & & $\square$ & \\
\hline Baghlan & [ & [ & 口 & & $\mathbf{\square}$ & [ & a & & [ & a & a \\
\hline Burka & & [ & a & & $\mathbf{\square}$ & & & & $\boldsymbol{\square}$ & ש & \\
\hline Dahanae G. & & & & $\square$ & $\mathbf{\square}$ & & & & 口 & a & \\
\hline Doshi & & 口 & & $\square$ & $\mathbf{\square}$ & & & & & $\mathbf{\square}$ & [ \\
\hline Khenjan & & & & & $\mathbf{\square}$ & & & & & D & \\
\hline Khost Fr & & a & 口 & & $\square$ & & & & & $\square$ & \\
\hline Nahrein & & & 口 & & $\square$ & & & & $\mathbf{a}$ & a & [ \\
\hline Puli Khum. & $\mathbf{\square}$ & [ & $\mathbf{\square}$ & & $\mathbf{\square}$ & $\boldsymbol{\square}$ & & $\square$ & & 口 & a \\
\hline Tala Barf. & & & & & & & & & & & \\
\hline Balkh & & & & & & [ & & & [ & & 口 \\
\hline Char Bolak & & & & & & & & & a & & \\
\hline Char Kant & & & & & 口 & a & & & & & \\
\hline Cheintal & & & & & & [ & & & & & \\
\hline Dehdadi & & & & & a & $\square$ & & & 口 & & 口 \\
\hline Dowlatabad & a & $\mathbf{\square}$ & 口 & & & 口 & $\square$ & & [ & $\square$ & \\
\hline Keshende & & & & & & & & & & & \\
\hline Mazar Sha. & & $\square$ & $\mathbf{\square}$ & & $\mathbf{\square}$ & $\mathbf{\square}$ & & $\boldsymbol{\square}$ & $\boldsymbol{\square}$ & & $\boldsymbol{\square}$ \\
\hline Nahr Shahi & & & $\boldsymbol{\square}$ & & $\mathbf{\square}$ & & & & [ & & \\
\hline Sholgera & & & & & $\mathbf{\square}$ & & & & & & \\
\hline Shortepa & & & & & & & & & & & \\
\hline Bamyan & & & $\square$ & $\square$ & a & [ & & & & $\square$ & \\
\hline Kahmard & & & 口 & 口 & च & & & & & 口 & \\
\hline Panjab & [ & & & $\square$ & $\mathbf{a}$ & & & & [ & & \\
\hline
\end{tabular}




\begin{tabular}{|c|c|c|c|c|c|c|c|c|c|c|c|}
\hline DISTRICTS & \multicolumn{11}{|c|}{ SECTORS } \\
\hline & AGR I & CONS & EDUC & EPI & $\begin{array}{l}\text { HEAL } \\
\text { TH }\end{array}$ & $\begin{array}{l}\text { INCO } \\
\mathrm{ME}\end{array}$ & MINE & $\begin{array}{l}\text { RELI } \\
\text { EF }\end{array}$ & $\begin{array}{l}\text { SOCI } \\
\text { AL }\end{array}$ & VET & $\begin{array}{l}\text { WATE } \\
\mathrm{R}\end{array}$ \\
\hline \multicolumn{12}{|l|}{ Saighan } \\
\hline \multicolumn{12}{|l|}{ Shibar } \\
\hline Waras & & & & & & & & & & & \\
\hline \multirow{2}{*}{\multicolumn{12}{|c|}{$\begin{array}{c}\text { FARAH Yakowlang } \\
\text { Anar Dara }\end{array}$}} \\
\hline & & & & & & & & & & & \\
\hline \multicolumn{12}{|l|}{ Bakwa } \\
\hline \multicolumn{12}{|l|}{ Bala Balok } \\
\hline \multicolumn{12}{|l|}{ Farah } \\
\hline \multicolumn{12}{|l|}{ Farsi } \\
\hline \multicolumn{12}{|l|}{ Gulistan } \\
\hline \multicolumn{12}{|l|}{ Khak Safid } \\
\hline \multicolumn{12}{|l|}{ Lash Jawen } \\
\hline \multicolumn{12}{|l|}{ Purchaman } \\
\hline \multicolumn{12}{|l|}{ Qala Kah } \\
\hline \multicolumn{12}{|l|}{$\begin{array}{l}\text { Shindand } \\
\text { FARYAB }\end{array}$} \\
\hline \multicolumn{12}{|l|}{ Almar } \\
\hline \multicolumn{12}{|l|}{ Andkhoy } \\
\hline \multicolumn{12}{|l|}{ Belcheragh } \\
\hline \multicolumn{12}{|l|}{ Darzab } \\
\hline \multicolumn{12}{|l|}{ Dowlatabad } \\
\hline \multicolumn{12}{|l|}{ Khan Char. } \\
\hline \multicolumn{12}{|l|}{ Kohistan } \\
\hline \multicolumn{12}{|l|}{ Maymana } \\
\hline Pashtun $\mathrm{K}$. & & & & & & & & & & & \\
\hline
\end{tabular}




\begin{tabular}{|c|c|c|c|c|c|c|c|c|c|c|c|}
\hline \begin{tabular}{||l|} 
DISTRICTS \\
\end{tabular} & \multicolumn{11}{|c|}{ SECTORS } \\
\hline & AGR I & CONS & EDUC & EPI & $\begin{array}{l}\text { HEAL } \\
\text { TH }\end{array}$ & $\begin{array}{l}\text { INCO } \\
\mathrm{ME}\end{array}$ & MINE & $\begin{array}{l}\text { RELI } \\
\text { EF }\end{array}$ & \begin{tabular}{ll|} 
SOCI \\
AL
\end{tabular} & VET & $\begin{array}{l}\text { WATE } \\
\text { R }\end{array}$ \\
\hline Qarghan & & [ & & & & & & & & & \\
\hline Qaysawr & & & & & & & & & & & \\
\hline Shirin Ta. & & & $\square$ & & & & & & & & \\
\hline $\mathrm{Ab}$ Band & $\mathbf{\square}$ & $\mathbf{E}$ & $\square$ & 口 & $\square$ & & & & & $\square$ & a \\
\hline Andar & ש & 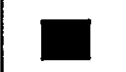 & 口 & $\square$ & $\mathbf{a}$ & & 口 & & [ & a & $\mathbf{\square}$ \\
\hline Deh Yak & a & a & 口 & 口 & $\square$ & & [ & & a & a & \\
\hline Gelan & E & $\overline{\mathbf{a}}$ & [ & a & a & & & & & a & $\mathbf{a}$ \\
\hline Ghazni & [ & D & 口 & 口 & $\square$ & a & $\mathbf{\square}$ & a & ש & $\square$ & a \\
\hline Giro & 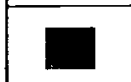 & $\mathbf{E}$ & & a & $\mathbf{0}$ & & & & a & $\mathbf{a}$ & a \\
\hline Jaghatu & $\bar{\square}$ & $\mathbf{E}$ & $\mathbf{a}$ & [ & a & ש & 口 & & [ & [ & \\
\hline Jaghori & $\square$ & a & a & 口 & [ & ש & & & 口 & a & a \\
\hline Khwaja Om. & $\square$ & 口 & 口 & [ & [ & & & & च & $\boldsymbol{\square}$ & (a) \\
\hline Malestan & a & [ & [ & [ & $\mathbf{\square}$ & & & & $\square$ & & \\
\hline Mogor & $\mathbf{\square}$ & 口 & 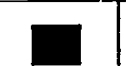 & a & 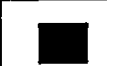 & & & & & $\square$ & $\square$ \\
\hline Nawa & D & [ & $\mathbf{\square}$ & $\square$ & a & & & & & [ & 口 \\
\hline Nawor & $\mathbf{\square}$ & & 口 & 口 & $\square$ & & & & & $\mathbf{E}$ & \\
\hline Qarabagh & $\mathbf{E}$ & & [ & [ & 口 & & ש & & [ & $\square$ & $\mathbf{a}$ \\
\hline Zena Khan & $\mathbf{\square}$ & 口 & $\square$ & 口 & a & $\mathbf{a}$ & & & [ & $\mathbf{\square}$ & \\
\hline Chaghcha. & 口 & & & & $\square$ & & & & & & \\
\hline Lal surj. & [ & & & $\square$ & $\square$ & & & & & & \\
\hline Pasaband & a & & & $\square$ & $\square$ & & & & & & \\
\hline Saghar & & & & & & & & & & & \\
\hline Shahrak & $\square$ & & & & & & & & & & \\
\hline Taywara & $\square$ & & & & & & & & & & \\
\hline Toulak & a & & & & & & & & & & \\
\hline
\end{tabular}




\begin{tabular}{|c|c|c|c|c|c|c|c|c|c|c|c|}
\hline DISTRICTS & \multicolumn{11}{|c|}{ SECTORS } \\
\hline \multirow{2}{*}{$\begin{array}{l}\text { HELMAND } \\
\text { Baghran }\end{array}$} & AGRI & CONS & EDUC & EPI & $\begin{array}{l}\text { HEAL } \\
\text { TH }\end{array}$ & $\begin{array}{l}\text { INCO } \\
\text { ME }\end{array}$ & MINE & $\begin{array}{l}\text { RELI } \\
\text { EF }\end{array}$ & $\begin{array}{l}\text { SOCI } \\
\text { AL }\end{array}$ & VET & $\begin{array}{l}\text { WATE } \\
\text { R }\end{array}$ \\
\hline & 口 & & & & $\square$ & & & & & & \\
\hline Bust & [ & $\square$ & & & [ & 口 & 口 & & $\mathbf{a}$ & [ & \\
\hline Deshu & & & & & a & & & & & & \\
\hline Garmser & a & & & & $\mathbf{a}$ & & & & & & [ \\
\hline Kajaki & ( & & & & $\square$ & & a & & & & \\
\hline Mosa Qala & a & & & & $\mathbf{\square}$ & & & & & & \\
\hline Nad-Ali & [ & & & a & $\square$ & & & & & & $\square$ \\
\hline Nahr Sar. & $\square$ & & & & 口 & [ & 口 & & & & 口 \\
\hline Nauzad & $\square$ & & & & a & & & & & & \\
\hline Nawae $\mathrm{Ba}$ & a & & & & $\square$ & & & & & & 口 \\
\hline Reg & [ & & & & 口 & & & & & & \\
\hline Sarban $Q$ & $\square$ & & & & D & & & & & & $\mathbf{\square}$ \\
\hline Washare & 口 & & & & 口 & & & & & & \\
\hline Adreskan & & 口 & & & & & & & & & \\
\hline Chesht Sha & & 口 & & & & & & & & & \\
\hline Enjeel & [ & $\mathbf{\square}$ & & & $\mathbf{a}$ & $\mathbf{a}$ & $\mathbf{\square}$ & & & 口 & $\square$ \\
\hline Ghoryan & [ & $\mathbf{\square}$ & & & & & & & & & a \\
\hline Gozarah & [ & [ & & & [ & [ & [ & & $\mathbf{0}$ & [ & $\square$ \\
\hline Gulran & [ & [ & & & & $\mathbf{\square}$ & & & & 口 & [ \\
\hline Herat & [ & 口 & D & [ & 口 & $\mathbf{a}$ & [ & [ & & & [ \\
\hline Karokh & & 口 & & & & Q & & & & $\boldsymbol{\square}$ & [ \\
\hline Kohsan & 口 & 口 & & & & & 口 & & & 口 & \\
\hline Kushk & [ & [ & $\mathbf{\square}$ & & & $\boldsymbol{\square}$ & [ & & & $\mathbf{E}$ & [ \\
\hline Obey & & $\square$ & & & & & & & & a & [ \\
\hline Pashtun $\mathrm{Z}$. & $\square$ & 口 & & & & & 口 & & & 口 & 口 \\
\hline Zendajan & $\square$ & a & & & $\square$ & & 口 & $\mathbf{a}$ & & a & \\
\hline
\end{tabular}




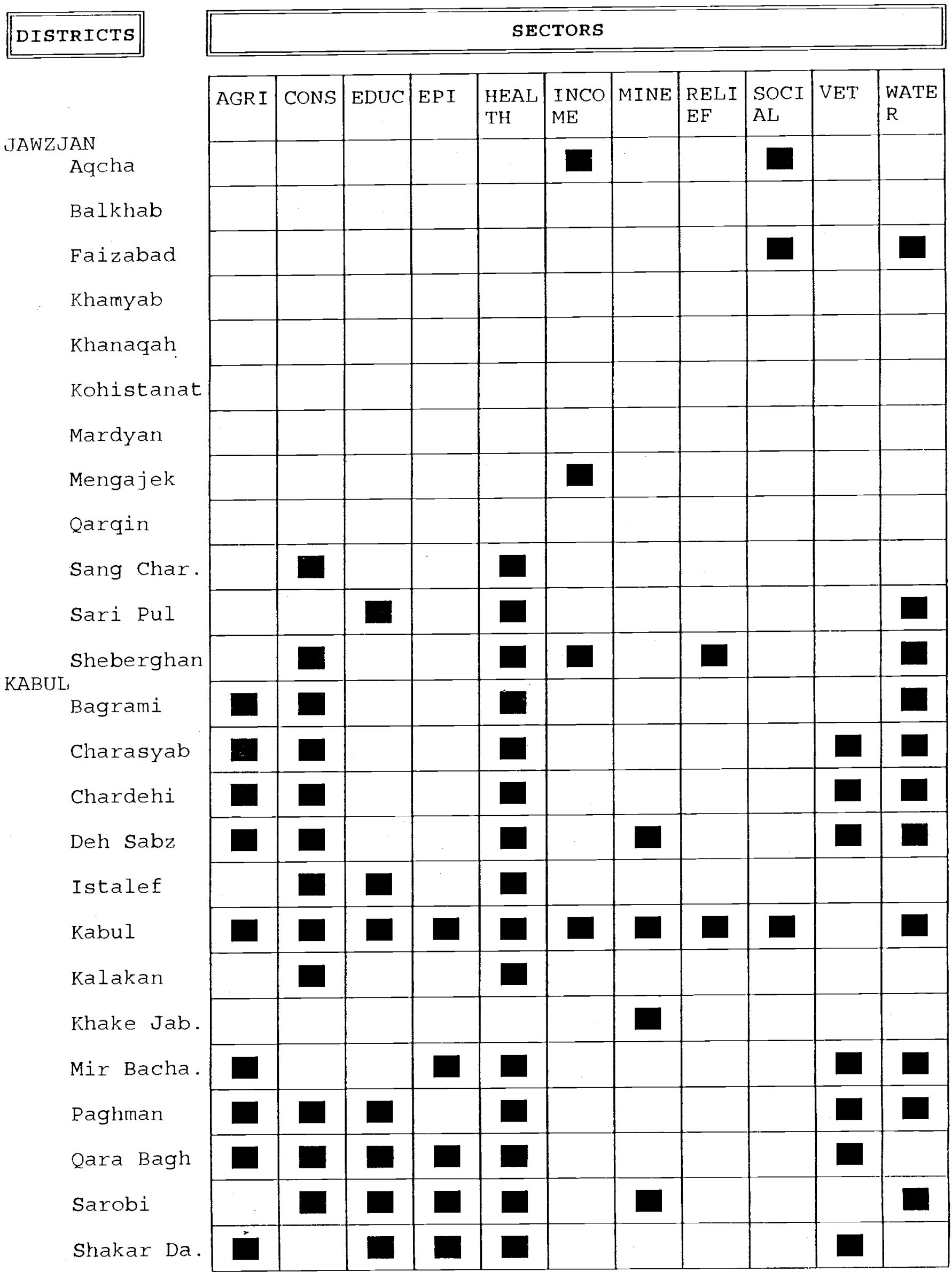




\begin{tabular}{|c|c|c|c|c|c|c|c|c|c|c|c|}
\hline \multirow[t]{2}{*}{ DISTRICTS } & \multicolumn{11}{|c|}{ SECTORS } \\
\hline & AGRI & CONS & EDUC & EPI & $\begin{array}{l}\text { HEAL } \\
\text { TH }\end{array}$ & $\begin{array}{l}\text { INCO } \\
\text { ME }\end{array}$ & MINE & \begin{tabular}{|l} 
RELI \\
EF
\end{tabular} & $\begin{array}{l}\text { SoCI } \\
\text { AL }\end{array}$ & VET & $\begin{array}{l}\text { WATTE } \\
\text { R }\end{array}$ \\
\hline $\begin{array}{l}\text { KANDAHAR } \\
\text { Arghandab }\end{array}$ & 口 & & & $\square$ & $\square$ & & $\mathbf{\square}$ & & & 口 & $\square$ \\
\hline Arghistan & 口 & $\mathbf{\square}$ & & & $\square$ & & 口 & & & & [ \\
\hline Daman & & $\square$ & & & $\square$ & 口 & $\mathbf{\square}$ & & & & $\mathbf{\square}$ \\
\hline D'and & 口 & $\mathbf{\square}$ & & $\mathbf{\square}$ & $\mathbf{\square}$ & & 口 & & & $\mathbf{a}$ & [ \\
\hline Ghorak & $\mathbf{\square}$ & & & & $\square$ & & & & & & \\
\hline Kandahar & $\mathbf{\square}$ & $\square$ & $\mathbf{\square}$ & $\square$ & $\square$ & $\mathbf{\square}$ & $\boldsymbol{\square}$ & & 口 & $\mathbf{\square}$ & $\mathbf{\square}$ \\
\hline Khakraiz & 口 & a & & a & $\mathbf{\square}$ & & & & & & \\
\hline Maruf & 口 & $\square$ & 口 & & [ & & & & & 口 & $\mathbf{a}$ \\
\hline Maywand & & $\square$ & & & $\square$ & & 口 & & & & $\mathbf{\square}$ \\
\hline Nesh & & & & & $\mathbf{a}$ & & & & & & \\
\hline Panjwai & $\mathbf{\square}$ & $\mathbf{\square}$ & & & G & & & & & & 口 \\
\hline Reg & & & & & 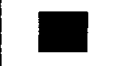 & & & & & & \\
\hline Shah W.K. & [ & $\mathbf{\square}$ & 口 & 口 & $\square$ & $\mathbf{\square}$ & & & & [ & $\mathbf{\square}$ \\
\hline Shega & & & & & $\square$ & & $\mathbf{a}$ & & & & $\mathbf{\square}$ \\
\hline Shorabak & & & & & $\mathbf{\square}$ & & & & & & $\mathbf{\square}$ \\
\hline$\underset{\text { Spin Bold. }}{\text { KAPISA }}$ & 口 & [ & & & [ & & & & & & $\mathbf{\square}$ \\
\hline Alasay & & & [ & & $\mathbf{\square}$ & & & & & & \\
\hline Kapisa & & & & $\mathbf{\square}$ & $\mathbf{\square}$ & & $\mathbf{\square}$ & & & 口 & \\
\hline Kohband & & & & & $\square$ & & & & & [ & \\
\hline Kohistan & & & & a & $\mathbf{\square}$ & & [ & & & $\mathbf{\square}$ & $\square$ \\
\hline Nejrab & & & $\mathbf{\square}$ & & $\square$ & & & & & [ & \\
\hline Panjshare & [ & [ & $\mathbf{\square}$ & & 口 & & & & & 口 & $\mathbf{\square}$ \\
\hline Panjshare 1 & & & & & $\mathbf{\square}$ & [ & & & & & \\
\hline Panjshare2 & & & & & $\mathbf{\square}$ & & & & & & \\
\hline Tagab & [ & $\square$ & 口 & & $\mathbf{\square}$ & & & & & & \\
\hline
\end{tabular}




\begin{tabular}{|c|c|c|c|c|c|c|c|c|c|c|c|}
\hline \multirow{2}{*}{ DISTRICTS } & \multicolumn{11}{|c|}{ SECTORS } \\
\hline & AGR I & CONS & EDUC & EPI & $\begin{array}{l}\text { HEAL } \\
\text { TH }\end{array}$ & $\begin{array}{l}\text { INCO } \\
\text { ME }\end{array}$ & MINE & $\begin{array}{l}\text { RELI } \\
\text { EF }\end{array}$ & $\begin{array}{l}\text { SOCI } \\
\text { AL }\end{array}$ & VET & $\begin{array}{l}\text { WATE } \\
\mathrm{R}\end{array}$ \\
\hline \multicolumn{12}{|l|}{ Bar Kunar } \\
\hline Barg Meta & & & & & & & & & & & \\
\hline Chaghasra & & & & & & & & & & & \\
\hline Chapa Dar & & & & & & & & & & & \\
\hline Chawki & & & & & & & & & & & \\
\hline Dongom & & & & & & & & & & & \\
\hline Kamdesh & & & & & & & & & & & \\
\hline Khas Kuna & & & & & & & & & & & \\
\hline Naranj/Ba & & & & & & & & & & & \\
\hline Naray & & & & & & & & & & & \\
\hline Nour Gul & & & & & & & & & & & \\
\hline Pech & & & & & & & & & & & \\
\hline Sirkanay & & & & & & & & & & & \\
\hline Aliabad & & & & & & & & & & & \\
\hline Archi & & & & & & & & & & & \\
\hline Char Dara & & & & & & & & & & & \\
\hline Hazrat Im & & & & & & & & & & & \\
\hline Khanabad & & & & & & & & & & & \\
\hline Kunduz & & & & & & & & & & & \\
\hline Qala Zal & & & & & & & & & & & \\
\hline Alingar & & & & & & & & & & & \\
\hline Alishing & & & & & & & & & & & \\
\hline Dawlatsha & & & & & & & & & & & \\
\hline Mehterlam & & & & & & & & & & & \\
\hline Nooristan & r & & & & & & & & & & \\
\hline Qarghaie & & & & & & & & & & & \\
\hline
\end{tabular}




\begin{tabular}{|c|c|c|c|c|c|c|c|c|c|c|c|}
\hline DISTRICTS & & & & & & TTORS & & & & & \\
\hline & AGRI & CONS & EDUC & EPI & $\begin{array}{l}\text { HEAL } \\
\text { TH }\end{array}$ & $\begin{array}{l}\text { INCO } \\
\mathrm{ME}\end{array}$ & MINE & $\begin{array}{l}\text { RELI } \\
\text { EF }\end{array}$ & $\begin{array}{l}\text { SOCI } \\
\text { AL }\end{array}$ & VET & $\begin{array}{l}\text { WATE } \\
\text { R }\end{array}$ \\
\hline $\begin{array}{l}\text { LOGAR } \\
\text { Azra }\end{array}$ & a & a & & & & & & & & $\square$ & \\
\hline Baraki & $\mathbf{E}$ & $\square$ & $\square$ & $\square$ & $\square$ & $\square$ & & & $\square$ & $\mathbf{\square}$ & $\mathbf{a}$ \\
\hline Charkh & $\mathbf{\square}$ & $\mathbf{0}$ & 口 & & [ & & & & a & $\square$ & $\square$ \\
\hline Khoshi & 口 & & $\mathbf{\square}$ & & [ & & [ & & & $\square$ & \\
\hline Mohd Agha & a & $\mathbf{\square}$ & 口 & & [ & & [ & & $\mathbf{\square}$ & $\mathbf{a}$ & a \\
\hline $\begin{array}{l}\text { Puli Alam } \\
\text { NANGARHAR }\end{array}$ & $\mathbf{a}$ & $\square$ & D & & a & $\square$ & 口 & & 口 & 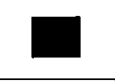 & \\
\hline Achin & 口 & & $\mathbf{\square}$ & & $\square$ & & [ & & & $\mathbf{\square}$ & 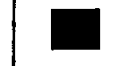 \\
\hline Bati Kot & 口 & 口 & $\mathbf{a}$ & & 口 & & & & & $\square$ & 1 \\
\hline Behsud & 口 & [ & a & & ש & a & $\mathbf{\square}$ & - & & $\square$ & 1 \\
\hline Chaparhar & $\square$ & & 口 & & D & & $\square$ & & & [ & \\
\hline Darae Noor & [ & [ & [ & & [ & & & & & & 口 \\
\hline Deh Bala & [ & [ & [ & & [ & & & & & [ & \\
\hline Dorbaba & & & & & [ & & & & & & \\
\hline Goshta & [ & & $\square$ & & $\mathbf{a}$ & & & & & 口 & $\square$ \\
\hline Hesarak & [ & $\square$ & $\mathbf{\square}$ & & $\mathbf{\square}$ & & [ & & & ב & \\
\hline Jalalabad & [ & [ & $\mathbf{a}$ & $\mathbf{a}$ & $\square$ & [ & $\mathbf{\square}$ & 口 & 口 & $\mathbf{a}$ & $\mathbf{a}$ \\
\hline Kama & [ & 口 & $\square$ & & $\square$ & & [ & & & $\mathbf{a}$ & a \\
\hline Khogiani & $\mathbf{a}$ & [ & [ & & $\square$ & & $\mathbf{\square}$ & & & $\square$ & a \\
\hline Kouz Konar & ש & a & $\mathbf{\square}$ & & $\mathbf{a}$ & [ & [ & 口 & & $\mathbf{\square}$ & a \\
\hline Lalpur & & & & & $\mathbf{\square}$ & & & & & $\mathbf{\square}$ & \\
\hline Mohmand $\mathrm{D}$. & 口 & 口 & [ & & [ & [ & & & & $\square$ & 口 \\
\hline Naziyan & & & $\mathbf{a}$ & & [ & & $\mathbf{a}$ & & & 口 & \\
\hline Pachier/A. & [ & [ & [ & & [ & & & & & 口 & \\
\hline Rodat & [ & [ & [ & & a & a & 口 & $\square$ & & $\square$ & a \\
\hline
\end{tabular}




\begin{tabular}{|c|c|c|c|c|c|c|c|c|c|c|c|}
\hline DISTRICTS & & & & & & CTORS & & & & & \\
\hline & AGRI & CONS & EDUC & EPI & $\begin{array}{l}\text { HEAL } \\
\text { TH }\end{array}$ & $\begin{array}{l}\text { INCO } \\
\text { ME }\end{array}$ & MINE & $\begin{array}{l}\text { RELI } \\
\text { EF }\end{array}$ & $\begin{array}{l}\text { SOCI } \\
\text { AL }\end{array}$ & VET & $\begin{array}{l}\text { WATE } \\
\text { R }\end{array}$ \\
\hline Sherzad & & & & & & & & & & & \\
\hline Shinwar & & H & & & 0 & & 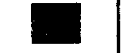 & & & & \\
\hline $\begin{array}{l}\text { Sorkh Rod } \\
\text { NIMROZ }\end{array}$ & & O & 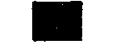 & & 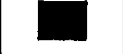 & $\square$ & L & & & & \\
\hline Asle Cha & & & & & & & & & & & \\
\hline Char Bor. & & & & & & & & & & & \\
\hline Kang/Kur. & & & & & & & & & & & \\
\hline Khosh Rod & & & & & & & & & & & \\
\hline Zaranj & & 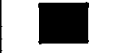 & & & & E & E & $\square$ & & & \\
\hline Ajrestan & & & & & & & & & & & \\
\hline Chorah & & & & & & & & & & & \\
\hline Dai Kundi & & & & & & & & & & & \\
\hline Deh Raud & & & & & & & 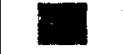 & & & & \\
\hline Gezab & & & & & & & & & & & \\
\hline Kajran & & & & & & & & & & & \\
\hline Oruzgan & & & & & & L & & & & & \\
\hline Shahrist. & & & & & & & & & & & \\
\hline Tirin & & & & & & $\square$ & $\square$ & & & & \\
\hline Barmal & & & & & & & & & & & \\
\hline Dila & & & & & & & & & & & \\
\hline Gayan & & & & & & & & & & & \\
\hline Gomal & & & & & & & & & & & \\
\hline Jani Khel & & & & & & & & & & & \\
\hline Katawaz & & & & E & - & & & & & & \\
\hline Mata Khel & & & & & & & & & & & \\
\hline
\end{tabular}




\begin{tabular}{|c|c|c|c|c|c|c|c|c|c|c|c|}
\hline \multirow[t]{2}{*}{ DISTRICTS } & \multicolumn{11}{|c|}{ SECTORS } \\
\hline & AGRI & CONS & EDUC & EPI & $\begin{array}{l}\text { HEAL } \\
\text { TH }\end{array}$ & $\begin{array}{l}\text { INCO } \\
\text { ME }\end{array}$ & MINE & $\begin{array}{l}\text { RELI } \\
\text { EF }\end{array}$ & $\begin{array}{l}\text { SOCI } \\
\text { AL }\end{array}$ & VET & $\begin{array}{l}\text { WATE } \\
\text { R }\end{array}$ \\
\hline Neka & $\mathbf{a}$ & & $\mathbf{\square}$ & & a & & & & & $\mathbf{\square}$ & \\
\hline Omna & & & & & 口 & & & & & $\mathbf{\square}$ & \\
\hline Sarhawza & [ & [ & & & & & & & & $\square$ & \\
\hline Sarobi & a & & D & & & & & & & $\mathbf{\square}$ & \\
\hline Sharan & & & [ & & 口 & & & & & 口 & \\
\hline Sultan Kh. & 口 & & a & & & & & & & $\mathbf{a}$ & [ \\
\hline Urgoun & 口 & [ & $\square$ & $\mathbf{0}$ & a & & & & & $\mathbf{a}$ & 口 \\
\hline Wazakhwa & & & $\mathbf{\square}$ & $\mathbf{a}$ & 口 & & & & & $\mathbf{a}$ & \\
\hline Wol-Mamay & & & & & & & & & & $\mathbf{\square}$ & \\
\hline Yusuf KheL & & & [ & & $\mathbf{\square}$ & & & & & $\mathbf{\square}$ & \\
\hline $\begin{aligned} \text { Zelook } & \end{aligned}$ & [ & & & $\mathbf{\square}$ & [ & & & & & a & \\
\hline Bak & & & $\mathbf{\square}$ & $\mathbf{a}$ & 口 & & $\square$ & & & $\square$ & $\mathbf{\square}$ \\
\hline Chamkani & [ & & $\mathbf{\square}$ & $\mathbf{0}$ & 口 & & [ & & a & a & \\
\hline Dand Patan & [ & & $\mathbf{\square}$ & $\mathbf{a}$ & [ & & & & & a & \\
\hline Dara Dara. & & & & $\mathbf{\square}$ & & & & & & & $\mathbf{\square}$ \\
\hline Gardaiz & [ & $\mathbf{a}$ & $\mathbf{\square}$ & $\mathbf{E}$ & a & $\mathbf{a}$ & $\mathbf{\square}$ & & $\mathbf{\square}$ & $\mathbf{a}$ & [ \\
\hline Gurbuz & $\mathbf{\square}$ & & $\mathbf{\square}$ & $\mathbf{a}$ & & & & & & $\mathbf{\square}$ & [ \\
\hline Hasan Khel & 口 & & $\mathbf{\square}$ & $\mathbf{\square}$ & $\mathbf{\square}$ & & & & $\boldsymbol{\square}$ & & \\
\hline Jadran & & $\boldsymbol{\square}$ & a & $\square$ & [ & $\mathbf{\square}$ & & & & $\mathbf{a}$ & 口 \\
\hline Jaji & 口 & $\mathbf{a}$ & $\mathbf{D}$ & $\square$ & a & $\square$ & $\mathbf{0}$ & & a & [ & 口 \\
\hline Jaji Maid. & & $\square$ & [ & & $\mathbf{\square}$ & & 口 & & & & [ \\
\hline Jani Khel & $\mathbf{\square}$ & & $\mathbf{\square}$ & $\square$ & $\mathbf{a}$ & & & & & $\mathbf{\square}$ & $\mathbf{\square}$ \\
\hline Khost & [ & $\boldsymbol{\square}$ & $\mathbf{\square}$ & $\square$ & [ & $\mathbf{\square}$ & a & & [ & a & [ \\
\hline Lajmangal & 口 & & $\mathbf{\square}$ & $\mathbf{\square}$ & 口 & & & & & & \\
\hline
\end{tabular}




\begin{tabular}{|c|c|c|c|c|c|c|c|c|c|c|c|}
\hline DISTRICTS & \multicolumn{11}{|c|}{ SECTORS } \\
\hline & AGRI & CONS & EDUC & EPI & $\begin{array}{l}\text { HEAL } \\
\text { TH }\end{array}$ & $\begin{array}{l}\text { INCO } \\
\text { ME }\end{array}$ & MINE & $\begin{array}{l}\text { RELI I } \\
\text { EF }\end{array}$ & $\begin{array}{l}\text { SOCI } \\
\text { AL }\end{array}$ & VET & \begin{tabular}{|l|} 
WATE \\
R
\end{tabular} \\
\hline Mandozi & 口 & $\square$ & & $\square$ & & & a & & & $\square$ & a \\
\hline Mosa Khel & $\square$ & & $\mathbf{\square}$ & a & $\square$ & & & & & & $\mathbf{\square}$ \\
\hline Nader Shah & 口 & a & $\square$ & a & $\square$ & & & & & 口 & [ \\
\hline Qalandar & & & & D & & & & & & & \\
\hline Sabari & $\square$ & & $\square$ & $\mathbf{\square}$ & a & & $\mathbf{0}$ & & & $\square$ & a \\
\hline Sayed Kar. & $\square$ & & $\square$ & $\mathbf{a}$ & $\square$ & $\square$ & $\square$ & & $\square$ & $\square$ & a \\
\hline Shamal & 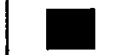 & & & $\mathbf{\square}$ & & & & & & & \\
\hline Shawak & a & & & a & & & & & & & \\
\hline Sperah & $\square$ & & $\square$ & $\boldsymbol{\square}$ & Q & & & & & & \\
\hline Tani & $\square$ & a & 口 & $\square$ & $\square$ & $\boldsymbol{\square}$ & a & & & [ & $\mathbf{\square}$ \\
\hline Trayzai & $\square$ & $\mathbf{\square}$ & 口 & $\square$ & $\square$ & & D & & & [ & $\mathbf{\square}$ \\
\hline Wolma & & & & $\mathbf{\square}$ & & & & & & & \\
\hline Zormat & [ & a & $\mathbf{\square}$ & $\mathbf{a}$ & $\mathbf{\square}$ & a & $\mathbf{a}$ & & & $\mathbf{\square}$ & $\square$ \\
\hline Bagram & $\square$ & $\square$ & & $\square$ & $\square$ & & D & & & $\mathbf{a}$ & $\square$ \\
\hline Charikar & $\square$ & $\square$ & $\square$ & $\square$ & $\square$ & $\mathbf{\square}$ & $\square$ & & & $\square$ & $\mathbf{\square}$ \\
\hline Ghorband & $\square$ & [ & $\square$ & $\square$ & $\mathbf{a}$ & & & & & $\square$ & a \\
\hline Jabul Sar. & $\square$ & $\square$ & $\mathbf{\square}$ & $\boldsymbol{\square}$ & $\square$ & & & & & $\square$ & \\
\hline Kohe Safi & & & & & $\square$ & & & & & D & \\
\hline Salang & & & [ & & 口 & & a & & & 口 & \\
\hline Shekh Ali & [ & & $\mathbf{\square}$ & $\boldsymbol{\square}$ & & & & & 口 & & \\
\hline Shenwari & & & & & 口 & & & & & $\square$ & \\
\hline Surkh Par. & & & $\mathbf{\square}$ & F & $\boldsymbol{\square}$ & & & & $\mathbf{\square}$ & $\mathbf{\square}$ & \\
\hline
\end{tabular}




\begin{tabular}{|c|c|c|c|c|c|c|c|c|c|c|c|}
\hline \begin{tabular}{|l||} 
DISTRICTS \\
\end{tabular} & \multicolumn{11}{|c|}{ SECTORS } \\
\hline & AGRI & CONS & EDUC & EPI & $\begin{array}{l}\text { HEAL } \\
\text { TH }\end{array}$ & $\begin{array}{l}\text { INCO } \\
\mathrm{ME}\end{array}$ & MINE & $\begin{array}{l}\text { RELI } \\
\text { EF }\end{array}$ & $\begin{array}{l}\text { SOCI } \\
\text { AL }\end{array}$ & VET & $\begin{array}{l}\text { WATE } \\
\text { R }\end{array}$ \\
\hline Dara Souf & & & $\mathbf{0}$ & & & & & & & & \\
\hline Kaldar & & & & & & [ & & & & & \\
\hline Hazrat S. & & & & & & & & & $\mathbf{\square}$ & & \\
\hline Khulm & & & $\mathbf{a}$ & & $\mathbf{\square}$ & & & & $\mathbf{\square}$ & & \\
\hline Roy Doab & & & 口 & & & & & & & & \\
\hline $\begin{array}{c}\text { Samangan } \\
\text { Sand }\end{array}$ & & & $\mathbf{\square}$ & & 口 & [ & 口 & & 口 & & 口 \\
\hline Bangi & & & & & a & & & & & $\mathbf{a}$ & $\square$ \\
\hline Chah Ab & & 口 & [ & & ( & & & & $\mathbf{\square}$ & a & $\mathbf{a}$ \\
\hline Chal & & & & & a & & & & & & \\
\hline Darqad & & $\boldsymbol{\square}$ & 口 & & 口 & & & & & 口 & \\
\hline Eshkamesh & & & [ & & 口 & & & & [ & ש & \\
\hline Farkhar & $\mathbf{\square}$ & & [ & & [ & & & & 口 & $\boldsymbol{0}$ & $\square$ \\
\hline Kalafgan & & $\mathbf{\square}$ & [ & & 口 & & & & $\mathbf{\square}$ & a & \\
\hline Khwajaghar & & & [ & & a & & & & [ & [ & \\
\hline Rustaq & & & $\mathbf{\square}$ & & 口 & & & & 口 & $\mathbf{\square}$ & \\
\hline Talogan & [ & 口 & $\mathbf{\square}$ & & [ & D & & & $\square$ & 口 & $\square$ \\
\hline Warsaj & & $\mathbf{\square}$ & $\mathbf{0}$ & & $\boldsymbol{\square}$ & & & & 口 & $\mathbf{\square}$ & \\
\hline $\begin{array}{c}\text { Yangi Qala } \\
\text { Yan }\end{array}$ & & $\mathbf{\square}$ & $\boldsymbol{\square}$ & & G & & & & $\mathbf{\square}$ & $\mathbf{a}$ & \\
\hline Chak & $\mathbf{\square}$ & $\mathbf{0}$ & $\boldsymbol{\square}$ & $\mathbf{\square}$ & $\mathbf{\square}$ & & & & $\mathbf{a}$ & $\mathbf{\square}$ & \\
\hline Day Mirdad & & & $\mathbf{\square}$ & 口 & $\boldsymbol{\square}$ & & & & a & a & \\
\hline Gardandewal & [ & & $\boldsymbol{\square}$ & & $\mathbf{0}$ & $\mathbf{a}$ & & $\mathbf{\square}$ & & a & \\
\hline Jaghatu & 口 & D & $\mathbf{a}$ & $\mathbf{\square}$ & $\mathbf{\square}$ & & & & a & $\mathbf{a}$ & $\mathbf{\square}$ \\
\hline Jalrez & [ & $\mathbf{\square}$ & $\mathbf{\square}$ & $\mathbf{\square}$ & $\mathbf{\square}$ & & & & & $\mathbf{a}$ & \\
\hline Markaz Bes & 口 & & $\mathbf{\square}$ & $\mathbf{\square}$ & $\mathbf{a}$ & $\mathbf{\square}$ & & $\square$ & ] & $\mathbf{a}$ & \\
\hline
\end{tabular}




\begin{tabular}{|c|c|c|c|c|c|c|c|c|c|c|c|}
\hline DISTRICTS & \multicolumn{11}{|c|}{ SECTORS } \\
\hline \multirow{3}{*}{$\begin{array}{l}\text { Maydan Shar } \\
\text { Nerkh }\end{array}$} & AGRI & CONS & EDUC & EPI & $\begin{array}{l}\text { HEAL } \\
\text { TH }\end{array}$ & $\begin{array}{l}\text { INCO } \\
\mathrm{ME}\end{array}$ & MINE & $\begin{array}{l}\text { RELLI } \\
\text { EF }\end{array}$ & $\begin{array}{l}\text { SOCI } \\
\text { AL }\end{array}$ & VET & $\begin{array}{l}\text { WATE } \\
\text { R }\end{array}$ \\
\hline & $\mathbf{\square}$ & $\mathbf{\square}$ & $\mathbf{0}$ & & [ & 口 & & & $\square$ & $\square$ & $\mathbf{\square}$ \\
\hline & $\mathbf{\square}$ & & a & & $\square$ & & & & [ & 口 & \\
\hline \multirow{2}{*}{$\begin{array}{l}\text { Syed Abad } \\
\text { ZABUL } \\
\text { Arghandab }\end{array}$} & $\mathbf{\square}$ & n & $\mathbf{\square}$ & $\square$ & $\square$ & 口 & & & 口 & $\square$ & a \\
\hline & & & & & 口 & & & & & & \\
\hline \multicolumn{12}{|l|}{ Atghar } \\
\hline \multirow{2}{*}{$\begin{array}{l}\text { Dai Chopan } \\
\text { Jaldak }\end{array}$} & $\boldsymbol{\square}$ & $\mathbf{\square}$ & & [ & $\mathbf{\square}$ & & & & & & \\
\hline & & & & & $\mathbf{\square}$ & & & & & & a \\
\hline \multicolumn{12}{|l|}{ Mizan } \\
\hline \multicolumn{12}{|l|}{ Qalat } \\
\hline \multirow{2}{*}{$\begin{array}{l}\text { Shah Joy } \\
\text { Shemalzai }\end{array}$} & & $\mathbf{a}$ & & & 口 & & $\mathbf{\square}$ & & & & $\mathbf{\square}$ \\
\hline & & & & & 口 & & & & & & \\
\hline \multicolumn{12}{|l|}{ Shinkai } \\
\hline Zabul & $\mathbf{\square}$ & & 口 & & & & & & $\mathbf{\square}$ & $\mathbf{\square}$ & $\mathbf{u}$ \\
\hline
\end{tabular}




\section{NUMBER OF NGOS IN DIFFERENT PROVINCES DURING 1995}

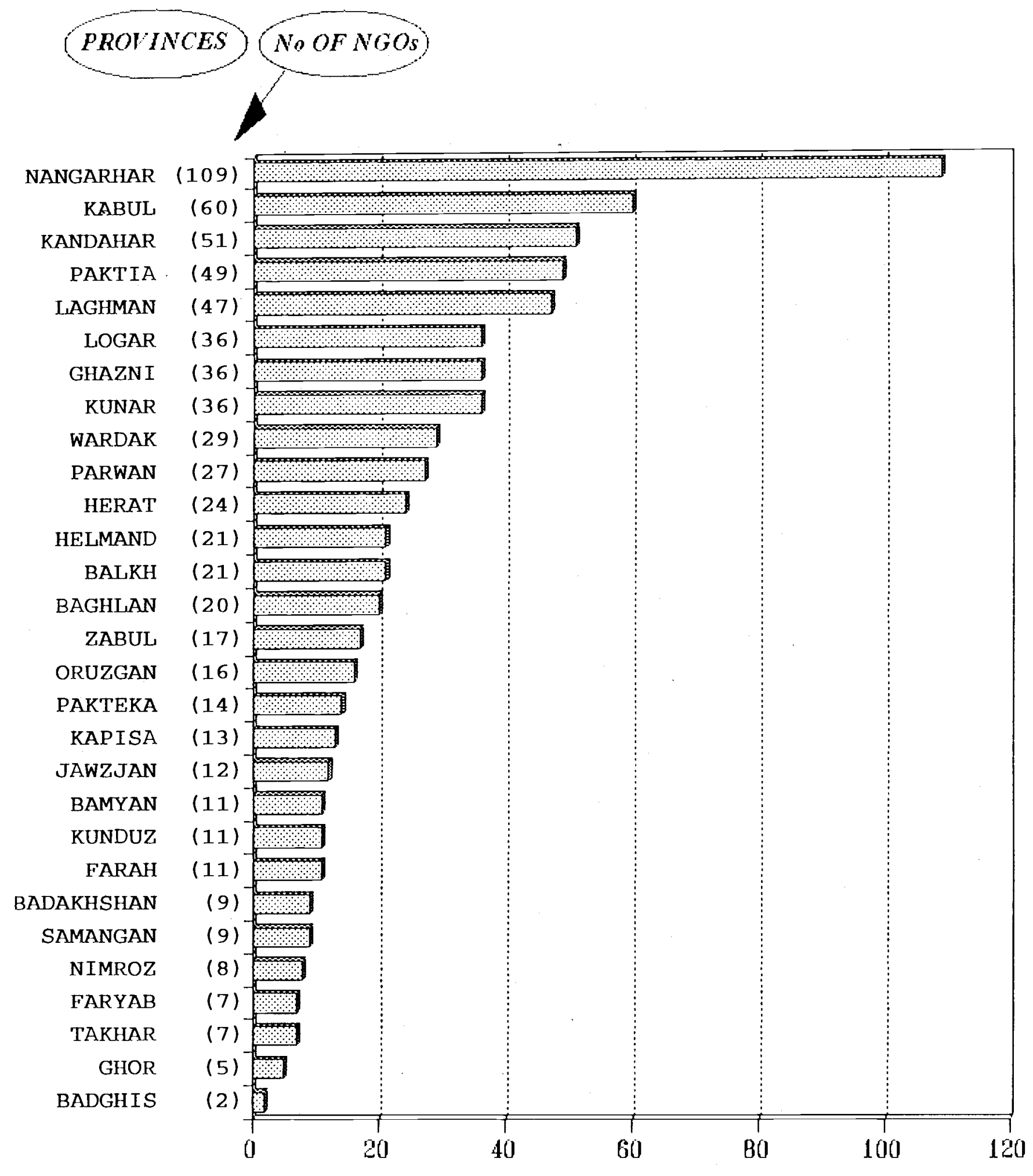


NGOs WORKING IN EACH DISTRICT DURING 1995

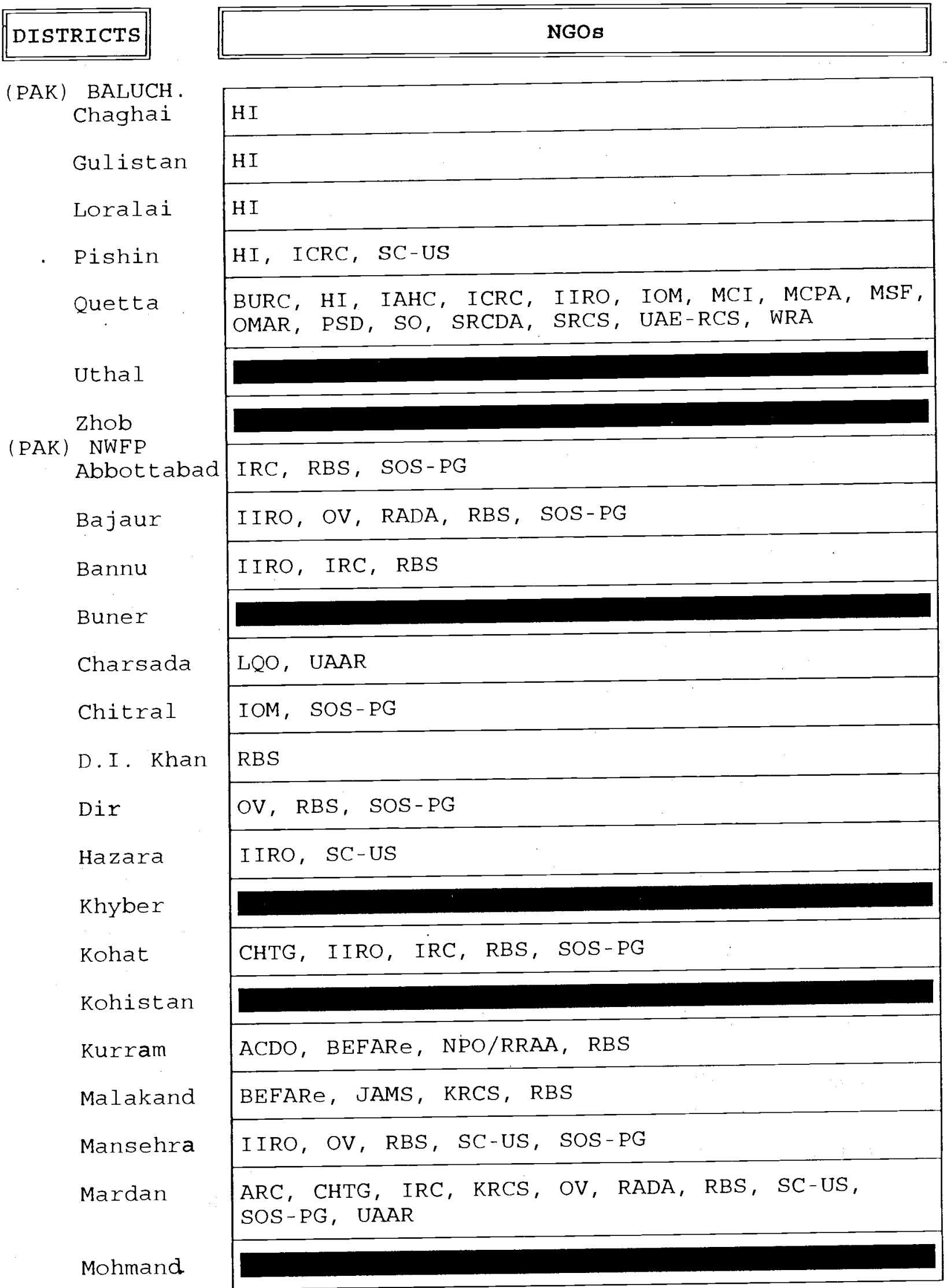




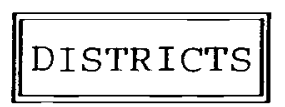

N. Wazir.

Nowshera

Orakzai

Peshawar

S.Wazir.

Swat

(PAK) PUNJAB

Is lamabad

Mian Wali

BADAKHSHAN

Baharak

Darwaz

Eshkashem

Faizabad

Jurm

Keranomon

Keshem

Khwahan

Ragh

Shar Boz.

Sheghnan

Wakhan

Zebak

BADGH IS

Ghormach

Jawand

Kushk Koh.

Morghab

Qades

Qala Nau

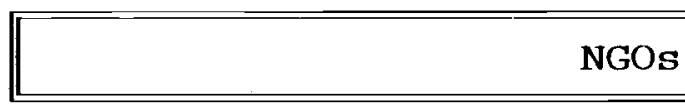

BEFARe，IRC， RBS

CARP, HCI, KRCS,

RBS

81 agencies

IIRO, IRC, RBS

RADA

AWEC, CBR, IIRO, VSO

CHTG， SC-US

A-AID， ARA，NAC， PRB， RAFA， SCA

$S C A$

A-AID， NAC， SCA

A-AID, ATC, NAC, PRB， SCA

A-AID, NAC, PRB， SCA

NAC， PRB， PRS， SCA

A-AID， NAC, SCA

NAC, SCA

A-AID, ORA, SCA

A-AID， NAC， SCA

PRB

FRF

FRF， IFRCS 


\section{DISTRICTS}

BAGHLAN

Anderab

Baghlan

Burka

Dahnae Gh.

Doshi

Khenjan

Khost Fr.

Nahrein

Puli Khum.

Tala Brfak BAI.KH

Balkh

Char Bolak

Char Kant

Chemtal

Dehdadi

Dowlatabad

Keshende

Mazar Shar

Nahr Shahi

Sholgera

BAMYAN

Bamyan

Kahmard

Panjab

Saighan
NGOB

ARA， DCA， SCA

A-AID, AACRP, CCA， GAA， IFRCS， MCPA， MSF， NADA， NPO/RRAA， OXFAM， SCA

DCA， RBS， SCA

AVICEN, DCA, IAM, SCA

AVICEN, DCA, IMC, PSD, SCA

DCA， SCA

DCA, SCA

DCA， NPO/RRAA， RBS， SCA

A-AID，AACRP，BRC，DCA， GP-UK， GRC， IFRCS， MSF， NPO/RRAA, OXFAM, SCA

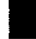

IAM, NPO/RRAA， OV, RBS

RBS

MSF， OV

CRFA, OV

OV, RBS, RDA, SCA

NPO/RRAA, OV, RBS, RDA

CCA, GRC, IAM, ICRC, IFRCS, MSF, NADA, OV, OXFAM, PSD, RBS, SC-US, SCA, SERVE， START，WRF

MAI， RBS, SCA

AVICEN, CCA, GAF, MAI, NADA, OXFAM， SCA

AVICEN， GAF， MAI， SCA

AVICEN, OXFAM

AVICEN, MAI 


\section{DISTRICTS}

Shibar

Waras

FARAH

Yakowlang

Anar Dara

Bakwa

Bala Baluk

Farah

Farsi

Gulistan

Khak Safid

Lash Jawen

Purchaman

Qala Kah

FARYAB

Shindand

Almar

Andkhoy

Belcheragh

Darzab

Dowlatabad

Khan Charb

Kohistan

Maymana

Pashtoon

Qaramqul

Qarghan

Qaysawr

Shirin Tag
AVICEN, CCA, GAF, MAI, SCA

AVICEN, OXFAM

AVICEN, CCA， GAF， OXFAM， PSD， SCA，SO，WRF

ARDA， AVICEN，CHA，FRF， MERLIN

FRF， MERLIN

CHA， FRF， MERLIN

ARDA， CHA，FRF， MDM， MERLIN, NADA， PSF， SWAARR

CHA

ARDA， FRF

ARDA，ARO，AVICEN， CHA，FRF， MERLIN

CHA，FRF， MERLIN

FRF

ARDA， CHA， MERLIN

ARDA, AVICEN, CHA, DACAAR, FRF, MERLIN, PSF

GRC， SC-US

\begin{tabular}{|l|}
\hline \\
\hline GRC \\
\hline SCA \\
\hline CCA, EDS, IFRCS \\
\hline EDS \\
\hline LAI \\
\hline
\end{tabular}




\begin{tabular}{|c|c|c|}
\hline DIS & RICTS & NGOs \\
\hline \multicolumn{3}{|c|}{$\begin{array}{l}\text { GHAZNI } \\
\qquad A b \text { Band }\end{array}$} \\
\hline & Andar & ADS, AFRANE, ATC, BDA, DACAAR, DCA, NAC \\
\hline & Deh Yak & ADS, AFRÁNE, AVICEN, DCA, LDI, MAI, MCPA \\
\hline & Gelan & AFRANE， AVICEN， COAR，GAF，SCA \\
\hline & Ghazni & $\begin{array}{l}\text { ACLU, ADS, AFRANE, ARO, ATC, AVICEN, BURC, CCA, } \\
\text { COAR, DACAAR, GAF, HAFO, HI, IAHC, ICRC, IFRCS, } \\
\text { ISRA， LDI, MAI, MCI, MSF, NAC, NADA, OMAR, RBS, } \\
\text { RDA, SCA }\end{array}$ \\
\hline & Giro & ADS, AFRANE, GAF, NAC, SCA \\
\hline & Jaghatu & AFRANE, COAR, DCA, MAI, NAC, NPO/RRAA, OMAR, RBS, SCA \\
\hline & Jaghori & AVICEN, GP-UK, GRSP, PSD, SCA, SO \\
\hline & KhwajaOmri & AFRANE, DACAAR, GAF, NAC, RBS, RDA， SCA \\
\hline & Malestan & AVICEN, GRSP, PSD, RDA, SCA, SO \\
\hline & Moqor & AFRANE, AVICEN, COAR, DCA, SCA, SOS-PG \\
\hline & Nawa & AFRANE, AVICEN, COAR, DCA, SCA \\
\hline & Nawor & AFRANE, AVICEN, DCA, SCA \\
\hline & Qarabagh & $\begin{array}{l}\text { ADS, ATC, AVICEN, DACAAR, DCA, PSD, RBS, RDA, } \\
\text { SCA, SO }\end{array}$ \\
\hline & Zena Khan & ADS, AFRANE, AVICEN, COAR, GAF, RBS, SCA \\
\hline & Chaghchran & ARO, ICRC, IFRCS \\
\hline & Lal Surj. & AVICEN, OXFAM \\
\hline & Pasaband & ARO, AVICEN \\
\hline & Saghar & \\
\hline & Shahrak & ARO \\
\hline & Taywara & ARO \\
\hline & Toulak & ARO \\
\hline
\end{tabular}




\section{DISTRICTS}

HELMAND

Baghran

Bust

Deshu

Garmser

Kajaki

Mosa Qala

Nad-Ali

Nahr Saraj

Nauzad

Nawae Bar.

Reg

Sarban Q.

Washare

HERAT

Adreskan

Chesht Sha

Enjee1

Ghoryan

Gozarah

Gulran

Herat

Karokh

Kohsan

Kushk

Obey

Pashtun $\mathrm{Za}$

Zendajan

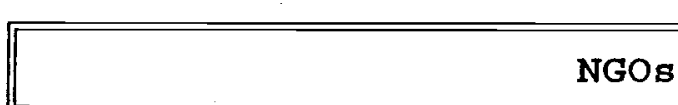

ARDA, AVICEN, HI

AFRC, ARDA, ARO, AURC, BURC, MCI, MCPA, MDC, MERLIN, MRORA, OMAR, RPSA

AVICEN

ARDA, AVICEN, VARA

ARDA, AVICEN, IAHC, MDC

ARDA， AVICEN， IAHC

ARDA, ARO, AVICEN, BURC, HWRA, KMA, RAFA

ARDA, AVICEN, MCPA， OMAR， RPSA

ARDA, AVICEN, HAFO

AROA， AVICEN， VARA

ARDA， AVICEN

ARDA， AVICEN， NERU

ARDA， AVICEN

I AM

DACAAR

ARDA, ARO, CHA， DACAAR， DCA， MCPA， MDC， MDC, NPO/RRAA， OMAR

DACAAR， FRF

ARDA， CHA， DACAAR， FRF， IAM， NPO/RRAA， OMAR

ARCAR， ARDA， DACAAR， FRF， NPO/RRAA

AITM, ARDA, AREA-1, CHA, IAM, ICRC, IFRCS, IIRO, IOM, MCPA, MDC, MERLIN, NADA， NPO/RRAA， OMAR, PSF， SCF-UK

CBR， DACAAR，DCA， NPO/RRAA

ARDA， DACAAR， DCA， MCPA， OMAR

ARDA， DACAAR，FRF， MCPA， NPO/RRAA， OMAR

DACAAR， DCA

ARDA， DACAAR， FRF， MCPA， NPO/RRAA， OMAR

ARDA， CHA， DACAAR，FRF， MCPA， NPO/RRAA， OMAR 
DISTRICTS

JAWZJAN

Aqcha

Balkhab

Faizabad

Khamyab

Khanaqah

Kohistanat

Mardyan

Mengajek

garqin

Sang Char.

Sari Pul

Sheberghan KABUL

Bagrami

Charasyab

Chardehi

Deh Sabz

Istalef

Kabul

Kalakan

Khake Jab.

Mir Bacha.

Paghman

Qara Bagh

Sarobi .

ShakarDara
CRFA， RBS

IAM

NGOs

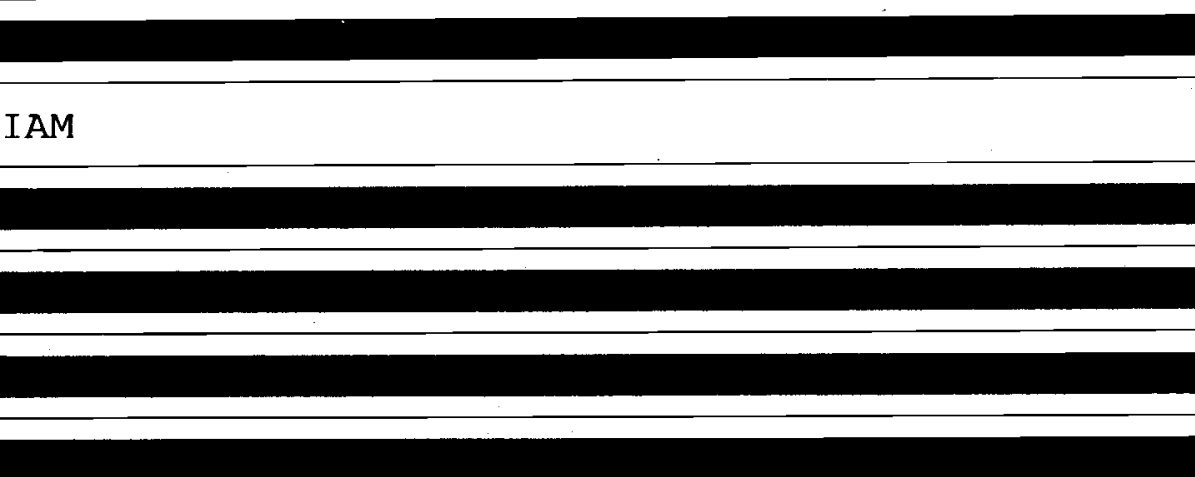

CRFA

GRC，MAI， SCA

ARCAR， GRC, MAI, SCA

IFRCS， NADA， OV， SERVE，WRF

ADAg, CARE, HCI， SCA， SJAWO

ARCAR, CARE, GAF， HCI， ICRC， MSF， RDM， SCA

CARE，GAF， RDA， SCA

ADAg, CARE, GAF, KPRO, MCPA, ORA， RAFA， SCA

IAM, MAI, SCA

AFRANE, AICF, AMI-A, ARCAR, ARCS, AREA-1, ARF, ATC, AVICEN, CCA, CHTG, CRFA, GAA, GP-UK, HT, IAM, RCRC, IFRCS, IMC, IOM, ISRA, JWMM, MARUF, MAWA, MCPA, MDC, MDM, MMC, MSF, MSOA, NADA, OMAR, ORA, OV, OXFAM, PDA, PRB, PSF, SAVE, SCA, SERVE, SJAWO, SO, SOLID, TDH, WRC

SCA

MCPA

ARPD， AVICEN， EMAR， GAF， SCA

AVICEN, CARE, GAF, ICRC, MAI, MSF, NAC, SCA

ARPD, AVICEN, GAF, ICRC, IMC, MAI, PSF, SCA

AAEA, AMRAN, ATC, AVICEN, ICRC, MCPA, MDC, SCA

AFRANE， AVICEN, CARE， GAF, MAI， PSF， SCA 
KANDAHAR

Arghandab

ARDA, ARIA, AVICEN, DACAAR, FRF, MDC, OMAR, SRO

Arghistan

AAA, AVICEN, EAFA, IAHC, MDC, MRORA， PETRA, SRA

Daman

ADA, ADO, AHDS, ARM, DACAAR, EAFA, HERI, HRAA, LKRO, MCPA, MDC, PETRA, SAMSOR, SRO

Dand

AHDS, ARDS, ARM, AURC, AVICEN, EAFA, FRF, KMA, MCPA, OMAR, RAFA, SAMSOR, SOLID, SWARO, VARA

Ghorak

ARDA, AVICEN, NERU

Kandahar

ACDA, ADA, ADARA, AHDS, AITM, ANH , ARDA, AVICEN, DACAAR, HRCA, I AHC, LKRO, MCI , MCPA, MDC, MERLIN, MRORA, NERO, NRO, OMAR, RAFA, RDA, SAMSOR, SOS-PG, SRCDA, SRRC, SWAARR, VARA

Khakraiz

AHDS, ARDA， AVICEN, PETER

Maruf

AVICEN， FRF， KMA， MRORA， SADA

Maywand

AVICEN, HERI， MCPA， MRC， OMAR

Nesh

Panjwai

AVICEN

AFRC, ARDA, ARM, AURC, AVICEN, HI, HRAA, HRCA, IAHC, LKRO, HERU, OHA, SRCDA

Reg

AVICEN

Shahwali K

ADA, AHDS, AVICEN, EAFA, FRF, HAFO

Shega

AVICEN, MCPA, MRC

Shorabak

AVICEN, NRO, SRO

Spin Bold.

KAPISA

\begin{tabular}{l|l} 
Alasay & \multicolumn{2}{|l}{ SCA } \\
\cline { 2 - 2 } Kapisa & AVICEN, GAF, PRB, SCA, SERVE \\
\cline { 2 - 2 } Kohband & GAF, SCA \\
\cline { 2 - 2 } Kohistan & ATC, AVICEN, GAF, PSF, SCA \\
\cline { 2 - 2 } Nejrab & GAF, MAI, SCA \\
\cline { 2 - 2 } Panjshare & A-AID, GAF, MAI, PRB, PSF, SCA \\
\cline { 2 - 2 } Panjshare1 & LDI, SCA \\
\cline { 2 - 2 } Panjare2 & MDM \\
\cline { 2 - 2 } Tagab & ADAg, PDA, SCA
\end{tabular}

ARDA, AURC, AVICEN, DARA, HERI, HRAA, LKRO, RAFA, RDA， SWAARR， SWARO， SCA 


\section{DISTRICTS}

KUNAR

Bar Kunar

Bargey Me.

Chaghasarai

Chapa Dara

Chawki

Dongom

Kamdesh

Khas Kunar

Naranj/Bad

Naray

Nour Gul

Pech

Sirkanay

\section{KUNDUZ}

Aliabad

Archi

Char Dara

Hazrat Im.

Khanabad

Kunduz

Qala Zal
NGOs

AINA, AMI-A, ANH, ATA/AP, DACAAR, ISRA, MADERA, SCA， SOS-PG

ATA/AP， WFA

AG-T'TP， AINA， ANH，ARCAR，ARDA， AREA-1，ATA/AP， AWC, BEFARe, DACAAR, ESAR, ISRA, LBI, MADERA, MAI, MF，MSF， NADA， NPO-RRAA，SCA， SERVE，SOS-PG，WRC

ANH, ARADA, ARDA, ATA/AP, BCURA, MADERA， SCA， SOS-PG，WRC

ATA/AP, ESAR, IIRO, ISRA, LQO, MADERA, MAI, SCA, SOS-PG, WRC,

$\mathrm{ANH}, \mathrm{ATA} / \mathrm{AP}$

AMI-A，ATA/AP， MADERA，MAI，SCA，SOS-PG，WRC

AG-TTP， ARDA，ATA/AP， HRP， IIRO， MAI， RAFA， SOS-PG

AMA， ANH, ARDA, ATA/AP, BEFARe, DACAAR, ESAR, ISRA， SCA， SOS-PG，WRC

ATA/AP, MADERA, SCA

ANH, ARDA, ATA/AP, HRP, MAI, RAFA， SCA， SOS-PG

AAID, AMA, ANH, ATA/AP, DACAAR, JAMS, MADERA, MAI， NAC， NPO/RRAA， SCA，SOS-PG， WRC

AMA, ARDA, ATA/AP, ISRA, MADERA, MAI, RSSA, SCA, SOS - PG

A-AID，PRB， RBS， SCA

PRB， SCA

$\mathrm{PRB}, \mathrm{SCA}$

$\mathrm{PRB}, \mathrm{SCA}$

A-AID， CBR， MSF， PRB， RBS， SCA

A-AID， CBR， IFRCS，IIRO， ISRA，KWA， MSF， NADA， $P R B ， S C A$

A-AID， PRB， SCA 


\section{DISTRICTS}

LAGHMAN

Al ingar

Alishing

Dawlatsha

Mehterlam

Nooristan

Qarghaie

\section{LOGAR}

Azra

Baraki

Charkh

Khoshi

Mohd Agha

Puli Alam

\section{NANGARHAR}

Achin

Bati Kot

Behsud

Chaparhar

Darae Noor

\section{NGOs}

ACDO, AMRAN, ANH, APWO, ARADA, ARC, BCURA, DACAAR, EMAR， GAF， IHSAN， JCE， LRO， MADERA， MAI， MARUF， MAWA, SCA

AMRAN, ANH, DACAAR, GAF, HCI , I IRO, LRO, MADERA, MAI， NPO/RRAA， RAFA， RDA， RDM， SCA

ARADA， BCURA， EMAR， GAF， MADERA， SCA

AFRO, AG-TTP, AITM, ANH, APWO, ARADA, DACAAR, EMAR, GAF, HCI， IFRCS, IIRO, ISRA, MADERA, MAI, MSF, NAC, NADA, NPO/RRAA, RAH, RSSA, SAVE, SCA, SERVE, SOS-PG， START， TAMIR， UMCA

ARDA， BDA， DACAAR， GAF， JAMS, MAI， SCA

AAEA, AMRAN, APWO, ARDA, AREA-1, ARD, DACAAR, ESAR, GAF, IHSAN, IIRO, ISRA, MADERA, MAI, NPO/RRAA， RSSA， SCA， SOS-PG，YARA

DCA， RDA，WRC

AFRANE, AMI-A， AWC， CARE， COAR， DACAAR， GAF, IRC, IRC/RPA， ISRA，PSF，RBS，RDA，RDW， SCA， SOS-PG

AFRANE, COAR, DACAAR, GAF， IRC， IRC/RPA， ISRA， MAI, RBS, SCA

ARC, DCA, IRC, IRC/RPA, MCPA, MDC, SCA

AFRANE, AFS, ARCAR, ATC, AWWO, CARE, GAF, IOC, IRC，IRC/RPA, MAI, MDC, MSF, RBS, SCA

AFRANE, AITM, ARA, AREA-1，ARO， AWWO, BONIAD, CARE, DCA, HCI， IIRO， IRC， IRC/RPA， ISRA， MAI, MCPA, MD, MSF, MDC, NADA, RADA, RBS, SCA

ARADA, ATC, LBI, MAI, MDC, MSF, SCA

AERRA， ARDA， DACAAR， IAU，ISRA，KNF， LBI， MSF， RAH, SCA, SOS - PG

AOGH, APWO, ARC, ARDA, ARDO, AREA-1, ATC, AVICEN, BCURA, DACAAR, EMAR, ICRC, IHSAN, ISRA, KNF, MADERA, MCPA, MDC, NPO/RRAA, RADA, RSSA, SCA, SERVE， SGRO， SJAWO， SOS-PG

ATC, BEFARe, DACAAR, GAF, I IRO, MCPA, OMAR, SCA, SOS - PG

ARDA, EMAR, GAF, HRP, ICRC, ISRA, JAMS, MADERA, ORA， SERVE， SOS-PG 


\begin{tabular}{|c|c|}
\hline DISTRICTS & NGOs \\
\hline Deh Bala & DCA, ICRC, ISRA, MAI, MAWA, SCA, SOS-PG, YARA \\
\hline Dorbaba & MSF \\
\hline Goshta & AAEA， ARDA， GAF， MADERA，SCA，SJAWO，SOS-PG， \\
\hline Hesarak & ADAg, AREA-1, DCA, MDC, SCA, SOS-PG \\
\hline Jalalabad & 76 agencies \\
\hline Kama & $\begin{array}{l}\text { AAA, ACDO, ACLU, ADAg, ARADA, ARDO, ARR, ATC, } \\
\text { DACAAR, EMAR, GAF, KNF, MADERA, RAFA， SCA, SOS-PG }\end{array}$ \\
\hline Khogiani & $\begin{array}{l}\text { AAA, ACDO, ACLU, AHSAO, AMRAN, ARDA, ATC, DACAAR, } \\
\text { DCA, IIRO, KNF, MARUF, MDC, SCA, SOS-PG }\end{array}$ \\
\hline Kouz Konar & $\begin{array}{l}\text { AMA, ARDA, ARIA, ATC, AVICEN, BEFARe, DACAAR, EMAR } \\
\text { GAF, MADERA, MCPA, RAH, RSSA, SERVE, SOS-PG }\end{array}$ \\
\hline Lalpur & ARDA, GAF, MSF, SCA \\
\hline Mohmand D. & $\begin{array}{l}\text { AAEA, ARC, DACAAR, GAF， ICRC， MSF， NPO/RRAA, } \\
\text { SOS-PG， YARA }\end{array}$ \\
\hline Naziyan & ARDA, ATC, MAI, MCPA, MDC, MF, MSF \\
\hline Pachier/Ag & ARPD， GAF，MAI， RSSA，SCA，SOS-PG \\
\hline Rodat & $\begin{array}{l}\text { ADAg, AERRA, AHDS, AOGH, ARADA, ARDA, AREA-1, } \\
\text { AREA-2, ARLO, ASDO, ATC, DACAAR, ERU, GAA, HAF, } \\
\text { I IRO, ASDO, ATC, DACAAR, ERU, GAA, HAF, IIRO, } \\
\text { IRC/RPA, ISRA, KNF, MADERA, MAI, MAWA, MCPA, MSF, } \\
\text { NPO/RRAA, RADA, SAA, SCA, SERVE, SOS-PG, SRDA }\end{array}$ \\
\hline Sherzad & ARDP, AREA-1， DCA，LBI， MAI， SCA \\
\hline Shinwar & ARADA, ARDA, ATC, IIRO, LBI, MCPA, MSF, RDA, SCA \\
\hline Sorkh Rod & $\begin{array}{l}\text { ACA, ACDO, AHSAO, AMRAN, ARDA, ARDO, ARF, ARIA, } \\
\text { ARR, ATC, AVICEN, BEFARe, DACAAR, GAA, GAF, IHSAN, } \\
\text { IIRO, KNF, MCPA, MDC, NCRA, NPO/RRAA, OMAR, PRS, } \\
\text { RSSA, SCA, SGRO, SOS-PG, WRC, YARA }\end{array}$ \\
\hline Asle Chak. & MCPA, VARA \\
\hline Char Borj. & FRF, VARA \\
\hline Kang/Kurki & VARA \\
\hline Khosh Rod & MCPA, VARA \\
\hline Zaranj & BURC, DARA， IFRCS， MCPA， MERLIN， NERU， VARA \\
\hline
\end{tabular}




\section{DISTRICTS}

ORUZGAN

Ajrestan

Chorah

Dai Kundi

Deh Raud

Gezab

Kajran

Oruzgan

Shahristan

Tirin

PAKTEKA

Barmal

Dila

Gayan

Gomal

Jani Khel

Katawaz

Mata Khel

Neka

Omna

Sarhawza

Sarobi

Sharan

Sultankhan

Urgoun

Wazakhwa

Wol-Mamay

Yusuf Khel

Zelook
NGOs

ADA

AVICEN

ADA, ARO, MCPA, MDC, MSF, OMAR, VARA

ADA

AVICEN, BURC

$\triangle \mathrm{DAA}, \mathrm{MCI}$

AVICEN， DCA， GRO

ADA, EAFA， IAHC, MCPA， MDC, MSF , OMAR, SOS-PG , SRCDA

DCA， SCA

NAC, SCA

$\mathrm{DCA}$

DCA， SCA

DCA，IRC， SCA

AVICEN， IRC，PRB， SCA

$\mathrm{PRB}, \mathrm{SCA}$

CARE， DCA， MAI， SCA

DCA, SCA

ARO, CARE, PRB

CARE， DCA， IRC， SCA

IRC，LDI， PRB， SCA

ARDP， IRC， IRC/RPA， PRB， WROR

AVICEN, CARE, IRC/RPA, MAI，NPO/RRAA，PRB，PRS, SCA

AVICEN， DCA， SCA

DCA

IRC, PRB， SCA

AVICEN, CARE， PRB， SCA 
PAKTIA

Bak

Chamkani

Dand Patan

DaraDarang

Gardaiz

Gurbuz

Hasan Khel

Jadran

Jaj i

JajiMaidan

Jani Khel

Khost

Lajmangal

Mandozi

Mosa Khel

Nader Shah

Qalandar

Sabari

SayedKaram

Shamal

Shawak

Sperah
APWO, ATC, DACAAR, GAF, IRC, IRC/RPA, MCAP, MDC, SCA

ADS, GAF, IRC, IRC/RPA, LBI, MAI, MCPA, SCA

GAF，IRC，IRC/RPA，SCA

IRC/RPA， SCA

ADS, AITM, ATC, CARE, DACAAR, IRC, IRC/RPA, ISRA, MCPA, MDC, MSF, NAC, NPO/RRAA, PRB， SCA

APWO， DACAAR，GAF，IRC， IRC/RRAA， MARUF， YARA

ADS, IRC/RPA, ISRA, MAI， SCA

CARE, GAF, IOC, IRC, IRC/RPA, MAI, SCA

AAA, ACRO, AG-TTP, AHSAO, AMRAN, ARDA, ARLO,ATC, GAF， HAFO， IRC/RPA，ISRA， LBI， MCPA， SCA

ATC, DACAAR, IRC, IRC/RPA，MCPA，MDC，SCA

GAF，IRC/RPA，MAI， SCA

AG-TTP, ARDP, AREA-1，ARO，ARR，ATC, AWC, BEFARe, CARE, DACAAR, ESAR, GAF, HCI, I IRO, IRC, IRC/RPA, ISRA, MAI, MCPA, MDC, NPO/RRAA, PRB， RDA， RDM, SCA, SJAWO, SNI, START

IRC，IRC/RPA， SCA

ATC, DACAAR, GAF, HCI, IRC, IRC/RPA, MCPA, SCA

IRC，IRC/RPA， SCA

AINA, CARE, DACAAR, GAF, HCI, IRC/RPA, SCA

IRC / RPA

ATC, GAF, IRC, IRC/RPA, MCPA, MDC, PRS, SCA

ADS, ARDP, ATC, IRC, IRC/RPA， ISRA， NPO/RRAA, SCA, WFA

IRC/RPA

IRC/RPA， ISRA

IRC，IRC/RPA，MAI，SCA， SJAWO 


\section{DISTRICTS}

Tani

Trayzai

Wolma

Zormat

PARWAN

Bagram.

Charikar

Ghorband

Jabulsaraj

Kohe Safi

Salang

Shekh Ali

Shenwari

SurkhParsa SAMANGAN

Dara Souf

Hazrat Sul

Kaldar

Khulm

Roy Doab

Samangan

TAKHAR

Bangi

Chah Ab

Chal

Darqad

Eshkamesh

\section{NGOs}

AHSAO, ATC, DACAAR, GAF, IRC/RPA, MAI, MARUF, MCPA， NPO/RRAA， PRS， SCA

AFS, APWO, ARF, ARR, GAF, IRC, IRC/RPA, ISRA, MCPA, MDC, RAFA， SCA

IRC / RPA

CARE， DACAAR， GAF，IRC，IRC/RPA， ISRA，MDC， NAC， NPO/RRAA， SCA

A-AID, AREA-1，ARPD， ATC, CBR， GAF， MCPA， PRB， RAFA， SCA

A-AID, AREA-1, ARPD, ATC, AVICEN, BDA, CARE, GAF, HT, IAM, ICRC, IFRCS, ISRA, MAI, MCPA, NADA, OMAR PRB， PSF，SCA， SERVE， SGAA， SOS-PG

A-AID, AVICEN, GAF， SCA

A-AID, ARA, AVICEN, CARE, CBR, DCA, PSF, SCA PRB， SCA

ATC， CBR， PRB， SCA

ARPD, AVICEN, DCA

DCA， SCA

AVICEN， DCA，PSD， SCA

MAI, SCA

RBS

CRFA

MAI， RBS， SCA

MAI

GAA，HT， IFRCS，NADA， RBS， SCA，WRF

A-AID， PRB， SCA

PRB， RBS， SCA

SCA

PRB， SCA

PRB， RBS， SCA 


\begin{tabular}{|c|c|}
\hline Farkhar & $A-A I D, P R B, \quad R B S, \quad S C A$ \\
\hline Kalafgan & PRB， RBS, SCA \\
\hline Khwajaghar & PRB， RBS， SCA \\
\hline Rustaq & PRB， RBS， SCA \\
\hline Taloqan & A-AID, ARA, MSF, NADA, PRB, RBS, SCA \\
\hline Warsaj & PRB， RBS， SCA \\
\hline Yangi Qala & PRB， RBS， SCA \\
\hline Chak & $\begin{array}{l}\text { ACRD, AFRANE, AVICEN, COAR, DCA, LDI, MAI, PSF, } \\
\text { RBS, SCA }\end{array}$ \\
\hline Day Mirdad & AVICEN, DCA, LDI, MAI, RBS, SCA \\
\hline Gardandewal & AVICEN, CAWC， DCA，MADERA， SCA \\
\hline Jaghatu & $\begin{array}{l}\text { ACRD, AVICEN, COAR, DACAAR, DCA, HAFO, MAI, PSF, } \\
\text { RBS, RDA, RDW, SCA, SOLID, SOS-PG }\end{array}$ \\
\hline Jalrez & ARCAR, AVICEN, DCA, LDI, MAI, SCA, SOLID \\
\hline Markaz Bes & AVICEN, CAWC, CCA, DCA, MADERA, RBS, SCA \\
\hline Maydanshar & CARE, DCA, MAI, NADA, RBS, SCA, SOLID \\
\hline Nerkh & CARE， DCA， RBS， SCA \\
\hline Syed Abad & $\begin{array}{l}\text { ACRO, ADA, AFRANE, AVICEN, CCA, COAR, DACAAR, } \\
\text { DCA, ESAR, IMC, ISRA, LBI, LDI, MAI, MSF, RBS, } \\
\text { RDA, RDW, SCA, SOS-PG }\end{array}$ \\
\hline Arghandab & AVICEN \\
\hline Atghar & \\
\hline Dai Chopan & AVICEN, PETRA \\
\hline Jaldak & AVICEN, VARA \\
\hline Mizan & AVICEN, KMA， MDC \\
\hline Qalat & $\begin{array}{l}\text { AÄA, ADA, AVICEN, MCI, MCPA, MDC, MERLIN, NADA, } \\
\text { OMAR, RAFA, SRA, SWAARR }\end{array}$ \\
\hline Shah Joy & AVICEN, HERI, OMAR, SRCDA \\
\hline Shemalzai & AVICEN \\
\hline Shinkai & AVICEN \\
\hline
\end{tabular}




\section{NUMBER OF NGOS IN DIFFERENT SECTORS IN PAKISTAN AND AFGHANISTAN DURING 1995}

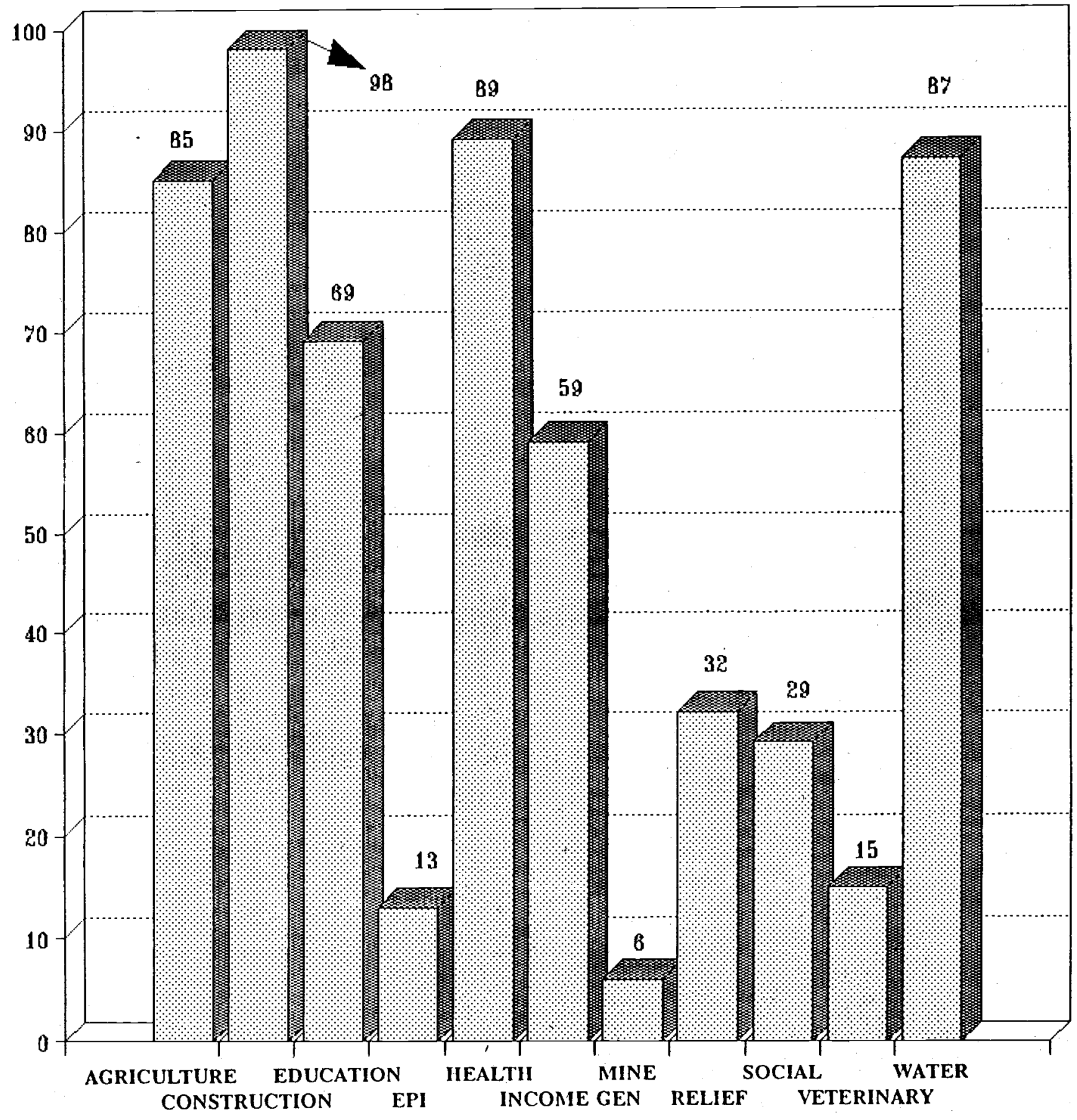


NGOS SECTOR OF WORK BY NGO DURING 1995

AGENCIES

SECTORS

A-AID

AAA

AABRAR

AACRP

AAEA

AAID

ACA

ACAA

ACDA

ACDO

ACLU

ACRD

ACRO

ADA

ADAg

ADARA

ADO

ADS

AERRA

AFRANE

AFRC

AFRO

AFS

AG-TTP

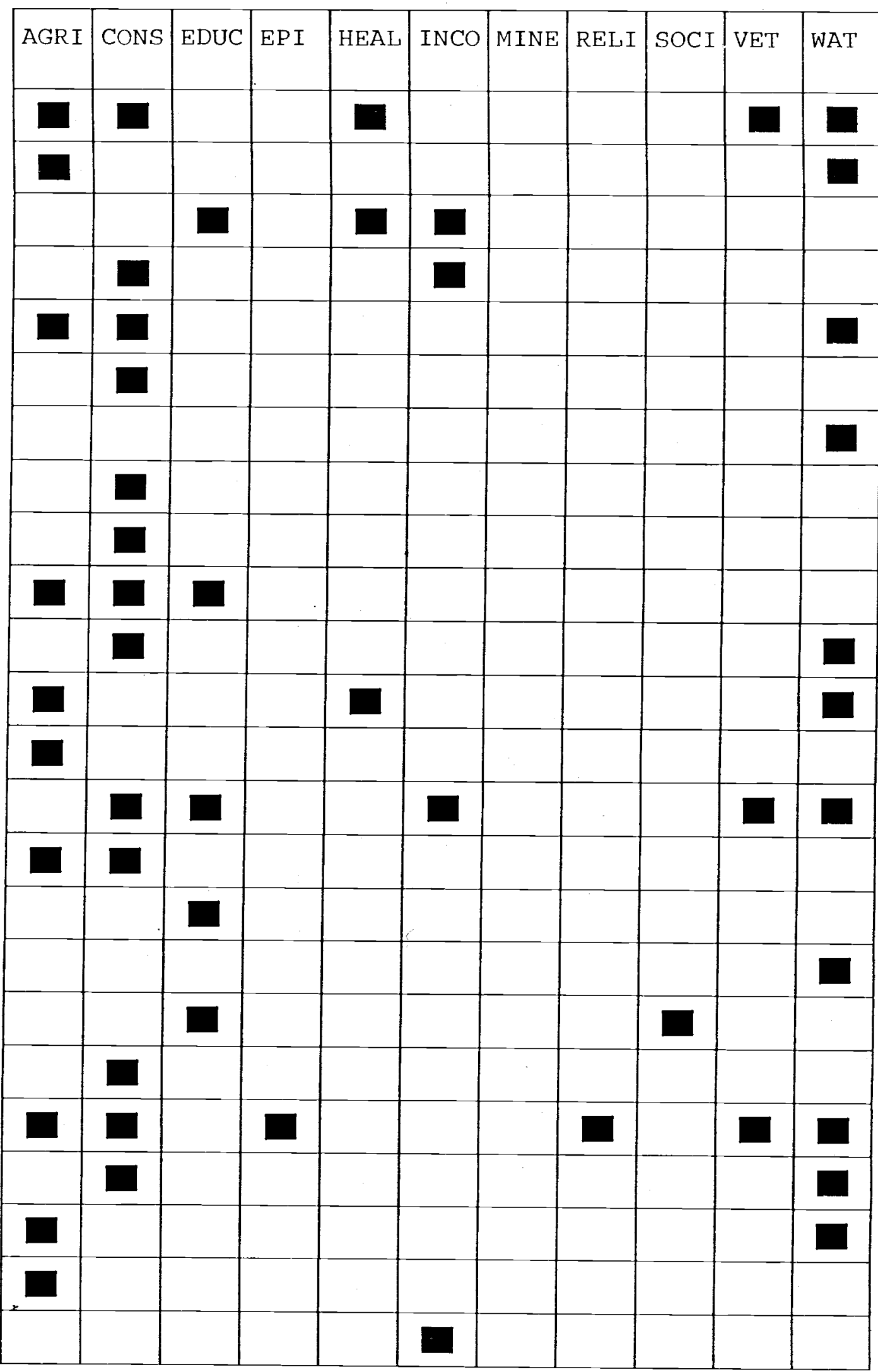


AHDS

AHSAO

AIC

AICC

AICF

AINA

AITM

AMA

AMI - A

AMRAN

AMRC

AMWS

ANAR

ANH

AOGH

APWO

ARA

ARAD

ARADA

ARC

ARCAR

ARCC

ARCON

ARCS

ARDA

\begin{tabular}{|c|c|c|c|c|c|c|c|c|c|c|}
\hline AGR I & CONS & EDUC & EPI & HEAL & INCO & MINE & RELI & SOCI & VET & WAT \\
\hline & & & & & & & & & & \\
\hline & & & & & & & & & & \\
\hline & & & & & & & & & & \\
\hline & & & & & & & & & & \\
\hline & & & & & & & & & & \\
\hline & & & & & & & & & & \\
\hline & & & & & & & & & & \\
\hline & & & & & & & & & & \\
\hline & & & & & & & & & & \\
\hline & & & & & & & & & & \\
\hline & & & & & & & & & & \\
\hline & & & & & & & & & & \\
\hline & & & & & & & & & & \\
\hline & & & & & & & & & & \\
\hline & & & : & & & & & & & \\
\hline & & & & & & & & & & \\
\hline & & & & & & & & & & \\
\hline & & & & & & & & & & \\
\hline & & & & & & & & & & \\
\hline & & & & & & & & & & \\
\hline & & & & & & & & & & \\
\hline & & & & & & & & & & \\
\hline & & & & & & & & & & \\
\hline & & & & & & & & & & \\
\hline & & & & & & & & & & \\
\hline
\end{tabular}




\begin{tabular}{|c|c|c|c|c|c|c|c|c|c|c|c|}
\hline AGENCIES & \multicolumn{11}{|c|}{ SECTORS } \\
\hline & AGRI & CONS & EDUC & EPI & HEAL & INCO & MINE & RELI & SOCI & VET & WAT \\
\hline ARDCD & & & & & & & & & & & \\
\hline ARDO & & & & & & & & & & & \\
\hline ARDP & & & & & & & & & & & \\
\hline AREA-1. & & & & & & & & & & & \\
\hline AREA - 2 & & & & & & & & & & & \\
\hline ARF & & & & & & & & & & & \\
\hline ARIA & & & & & & & & & & & \\
\hline ARLO & & & & & & & & & & & \\
\hline ARM & & & & & & & & & & & \\
\hline ARO & & & & & & & & & & & \\
\hline ARPD & & & & & & & & & & & \\
\hline ARR & & & & & & & & & & & \\
\hline ARRAO & & & & & & & & & & & \\
\hline ASDO & & & & & & & & & & & \\
\hline ATA/AP & & & & & & & & & & & \\
\hline ATC & & & & & & & & & & & \\
\hline AURC & & & & & & & & & & & \\
\hline AVICEN & & & & & & & & & & & \\
\hline AWC & & & & & & & & & & & \\
\hline AWEC & & & & & & & & & & & \\
\hline AWRC & & & & & & & & & & & \\
\hline AWWD & & & & & & & & & & & \\
\hline AWWO & & & & & & & & & & & \\
\hline BCURA & & & & & & & & & & & \\
\hline BDA & & & & & & & & r & & & \\
\hline
\end{tabular}


BEFARe

BONIAD

BRC

BURC

CARE

CARP

CAWC

CBR

CCA

CHA

CHTG

COAR

CRAA

CRFA

DACAAR

DARA

DCA

DgCAR

DS

EAFA

ECAR

EDS

EMAR

ERU

ESAR

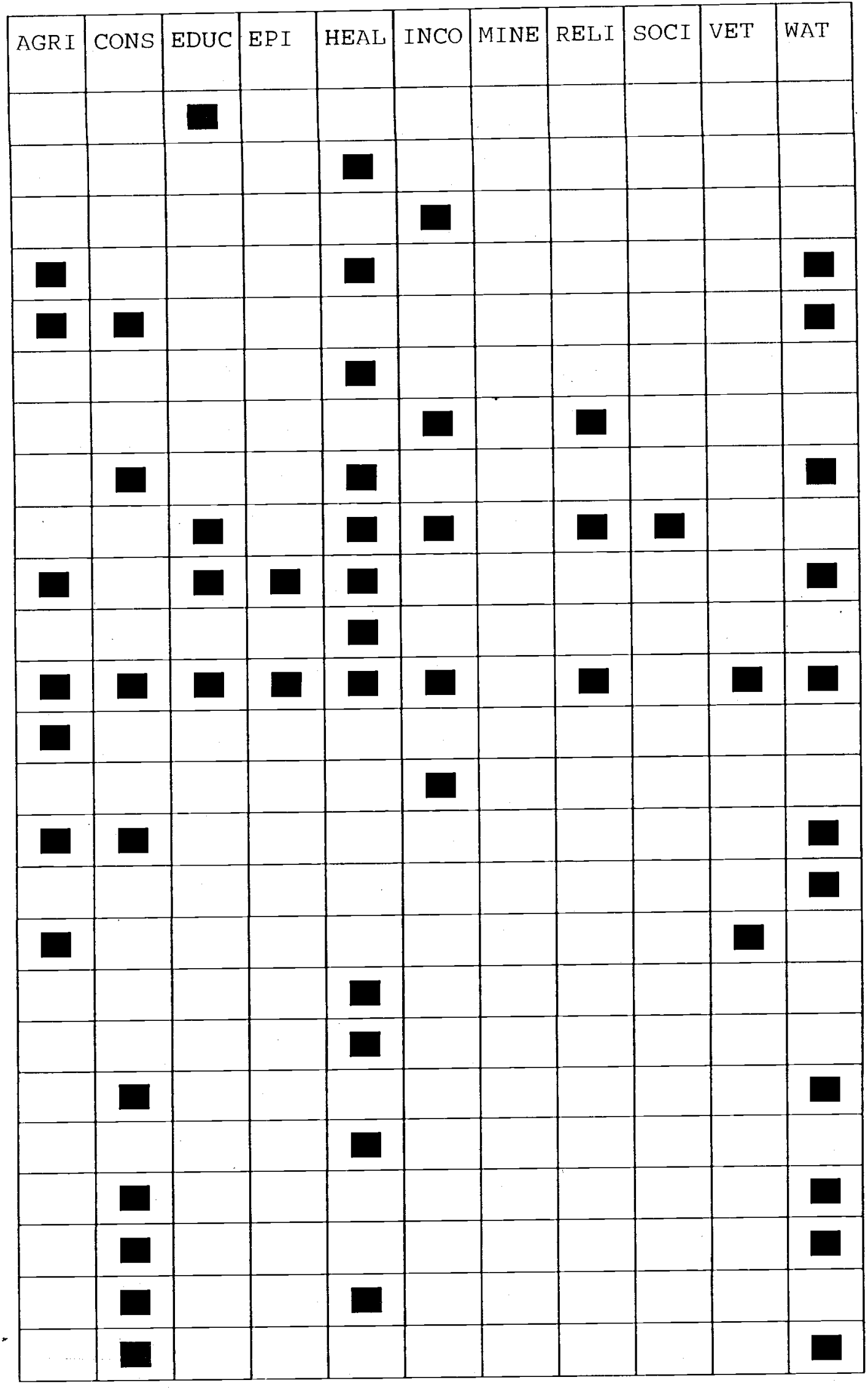


AGENCIES

FRF

GAA

GAF

GP - UK

GRC

GRSP

HAF

HAFO

HCI

HDCAW

HELP

HERI

HI

HRAA

HRCA

HRP

HT

HWRA

IAAAE

IAHC

IAM

IAU

ICD

ICRC

IFRCS
SECTORS

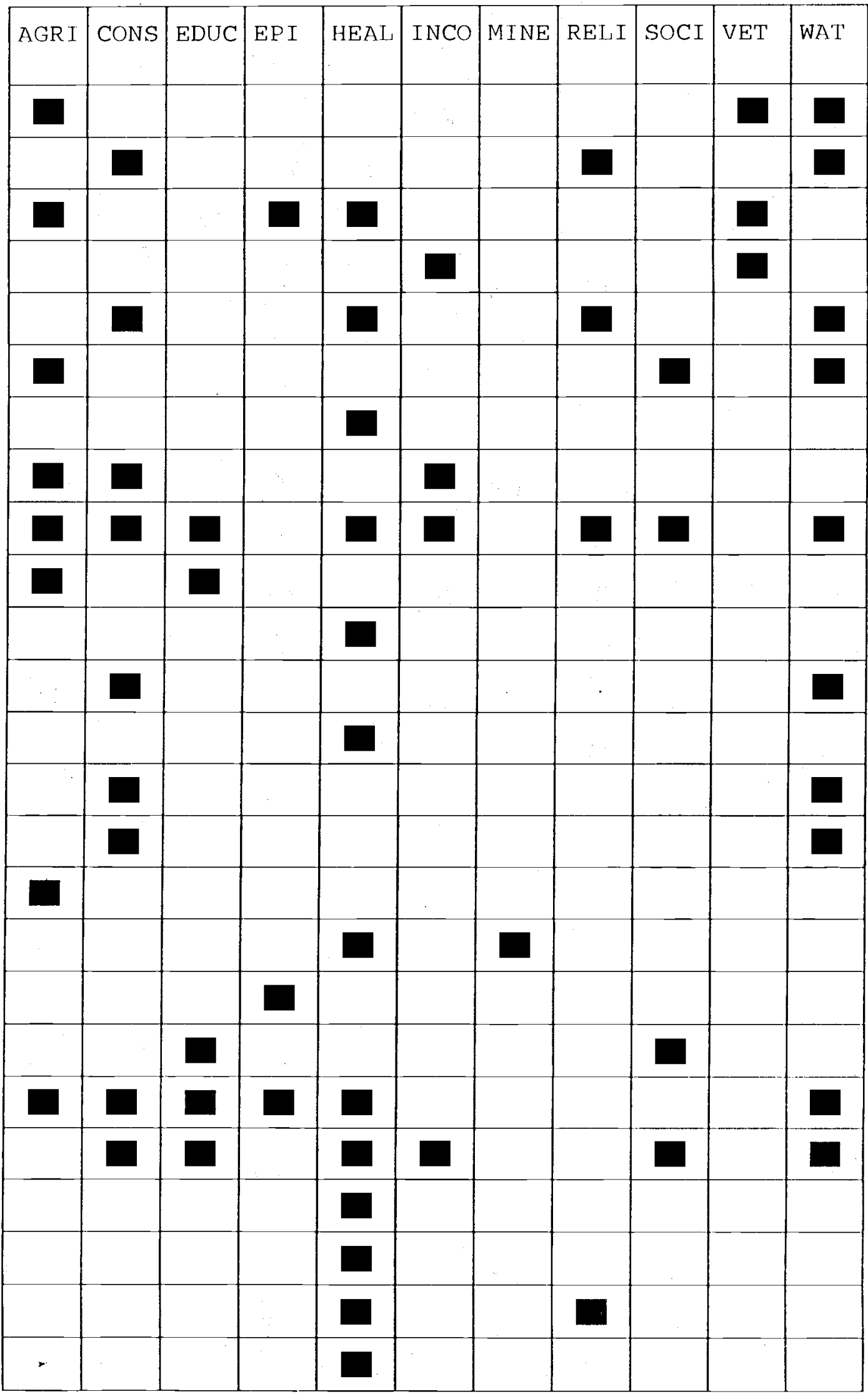


IHSAN

I I RO

IMC

IOC

IOM

IRC

IRC/RPA

ISRA

JAMS

JCE

JIFF

JWMM

KMA

KNF

KPRO

KRCS

KWA

LBI

LDI

LKRO

LQO

LRO

MADERA

MAI

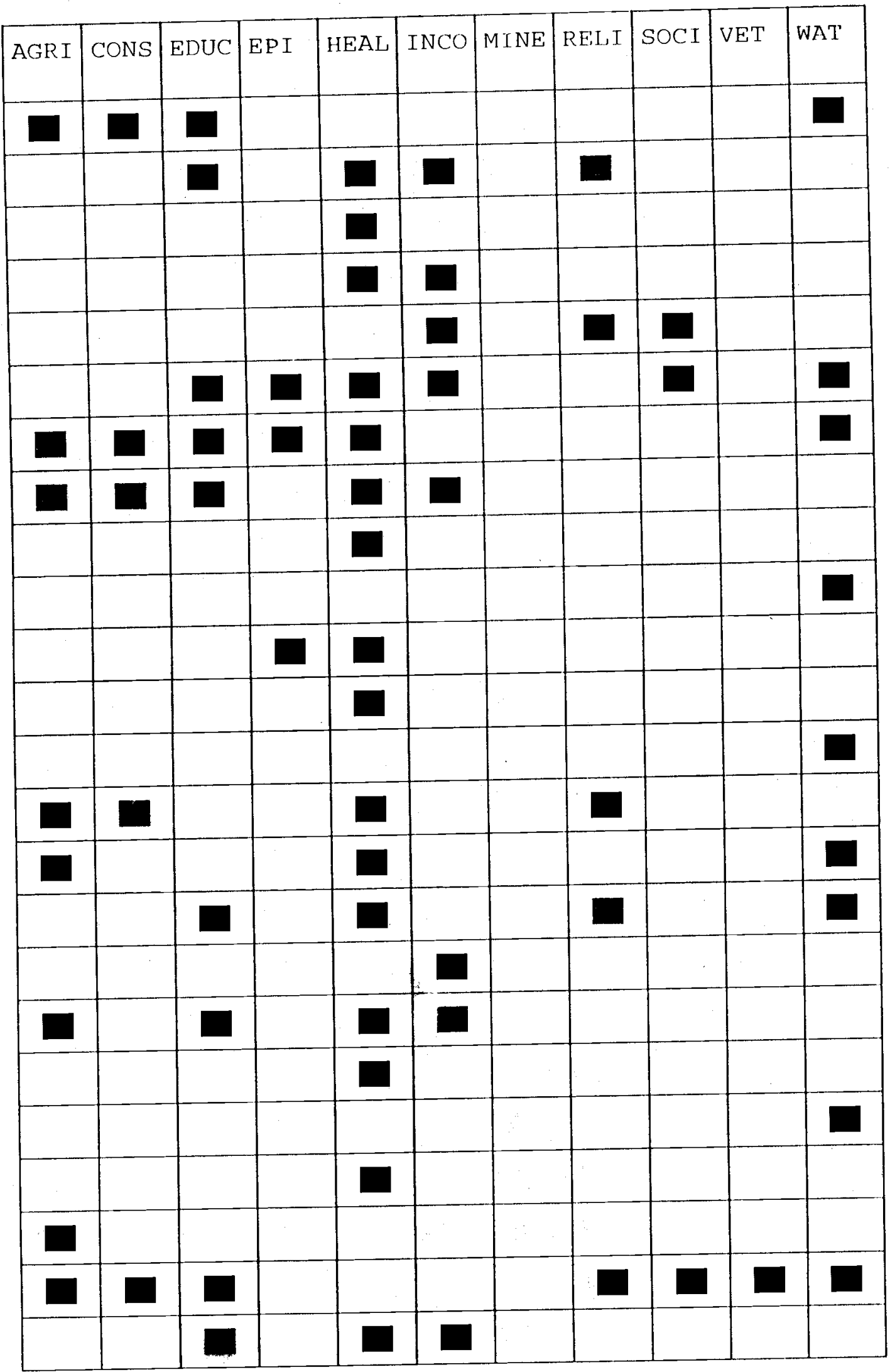


AGENCIES

MARUF

MAWA

MCI

MCPA

MDC

MDM

MERLIN

MF

MHC

MMC

MPO

MRC

MRCA

MRORA

MSF

MSOA

NAC

NADA

NCRA

NERO

NERU

NPO/RRAA

NRO

OHA

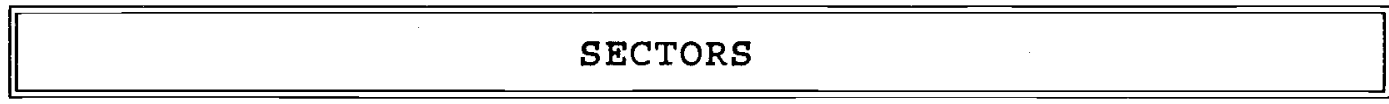

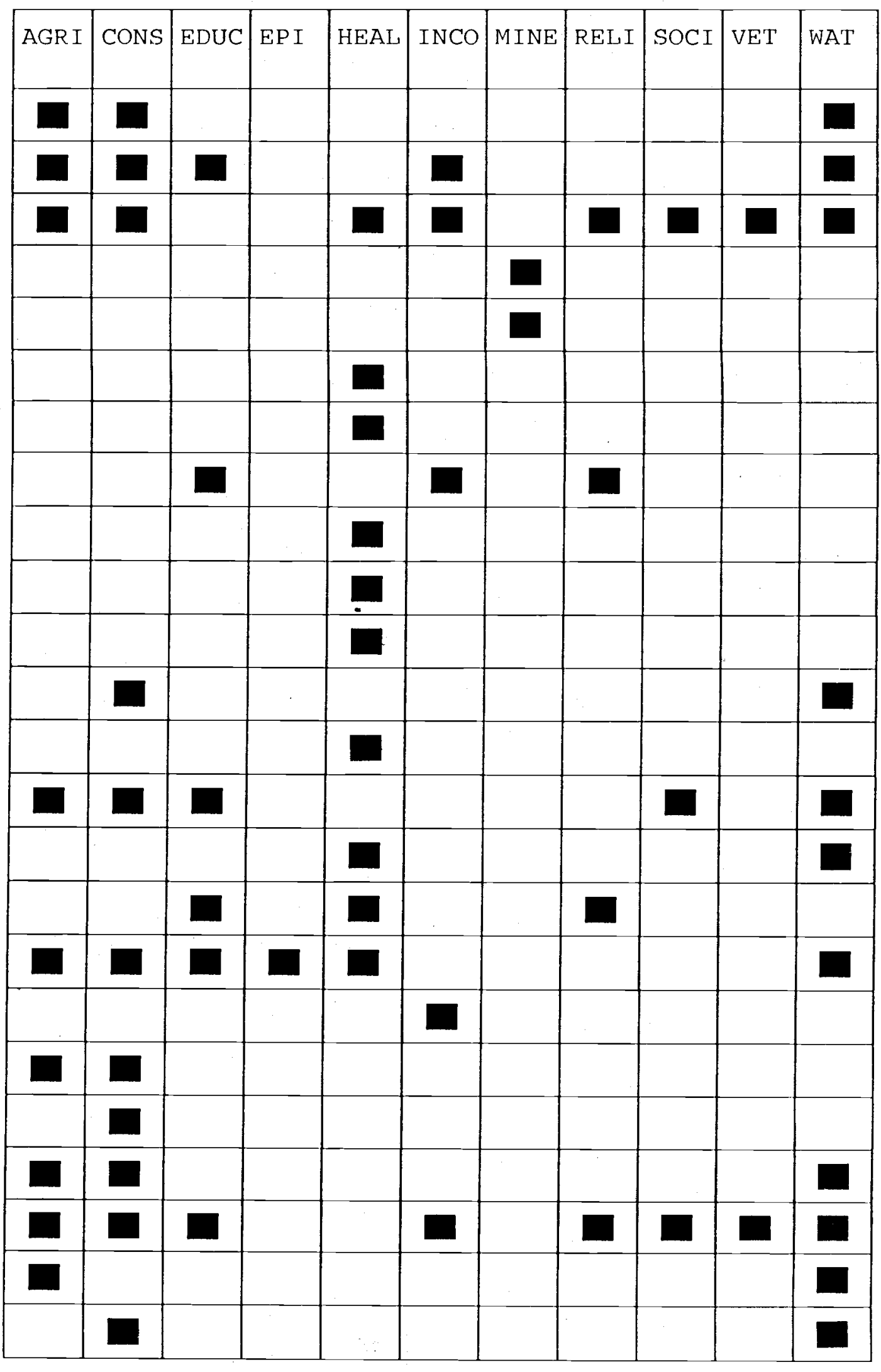


OMAID

OMAR

ORA

ov

OXFAM

PARDC

PDA

PETRA

PRB

PRCS

PRS

PSD

PSF

RADA

RAFA

RAH

RBS

RDA

RDM

RDW

ROAOW

RPA

RPSA

RSSA

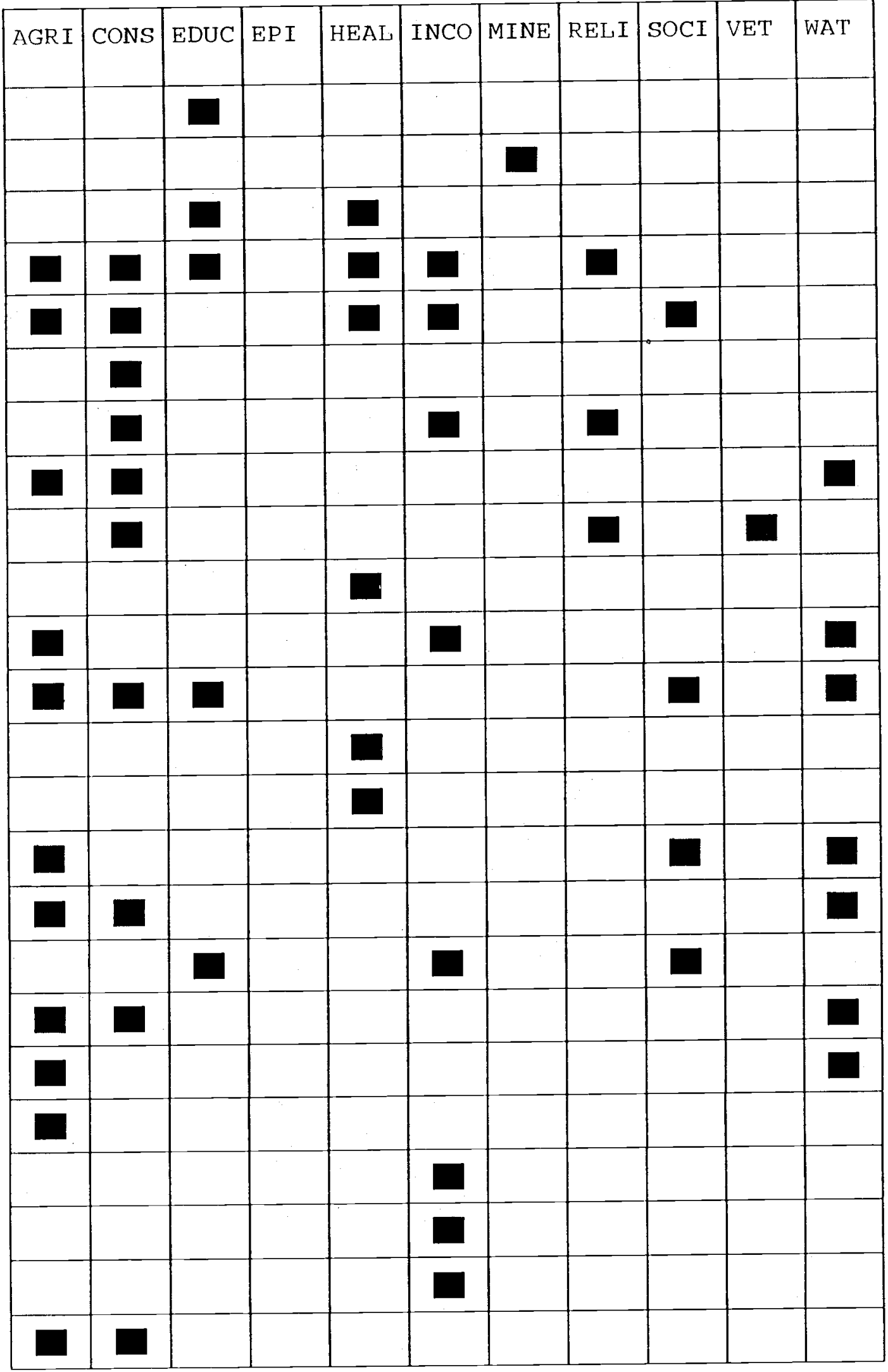




\begin{tabular}{|c|c|c|c|c|c|c|c|c|c|c|c|}
\hline AGENCIES & \multicolumn{11}{|c|}{ SECTORS } \\
\hline & AGRI & CONS & EDUC & EPI & HEAL & INCO & MINE & RELI & SOCI & VET & WA'T \\
\hline SAA & & & 口 & & & & & & & & \\
\hline SADA & & & & & & & & & & & \\
\hline SAMSOR & & & & & & & & & $\mathbf{\square}$ & & $\square$ \\
\hline SAVE & a & $\mathbf{\square}$ & & & & & & & 口 & & \\
\hline SC-US & & & $\square$ & & 口 & 口 & & & & & \\
\hline $\mathrm{SCA}$ & [ & $\mathbf{\square}$ & 口 & & 口 & & & & & & $\square$ \\
\hline SCF-UK & & $\square$ & [ & & 口 & & & & & & \\
\hline SERVE & 口 & $\square$ & & & a & & & $\square$ & & & $\mathbf{E}$ \\
\hline SGAA & & & & & $\mathbf{\square}$ & & & & & & \\
\hline SGRO & & [ & & & 口 & & & [ & & & \\
\hline SHACA & & & 口 & & & & & & & & \\
\hline SJAWO & $\mathbf{\square}$ & a & 口 & & & 口 & & $\square$ & & & \\
\hline SNI & & $\mathbf{\square}$ & & & & $\mathbf{\square}$ & & 口 & & & $\square$ \\
\hline so & & $\square$ & 口 & 口 & $\square$ & a & & 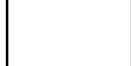 & & & \\
\hline SOLID & [ & $\mathbf{\square}$ & & & & & & $\mathbf{\square}$ & & & 口 \\
\hline SOS-PG & $\mathbf{\square}$ & & [ & & & 口 & & & & & \\
\hline SRA & & & & & & & & & & & $\square$ \\
\hline SRCDA & & [ & [ & & & & & & & & $\square$ \\
\hline SRCS & & a & $\square$ & & [ & & & & & & $\mathbf{\square}$ \\
\hline SRDA & & $\mathbf{\square}$ & . & & [ & & & & & & \\
\hline SRO & & & & & & & & & & & D \\
\hline SRRC & & 口 & & & & & & & & & \\
\hline START & a & 口 & 口 & & & [ & & & & & \\
\hline SWAARR & & & & & & & & & & & D \\
\hline SWARO & & & & & & & & & & & \\
\hline
\end{tabular}


TAMIR

TDH

TRO

UAAR '

UAE-RCS

UMCA

UNO / EPA

VARA

vSO

WFA

WRA

WRC

WRF

WROR

WUFA

YARA

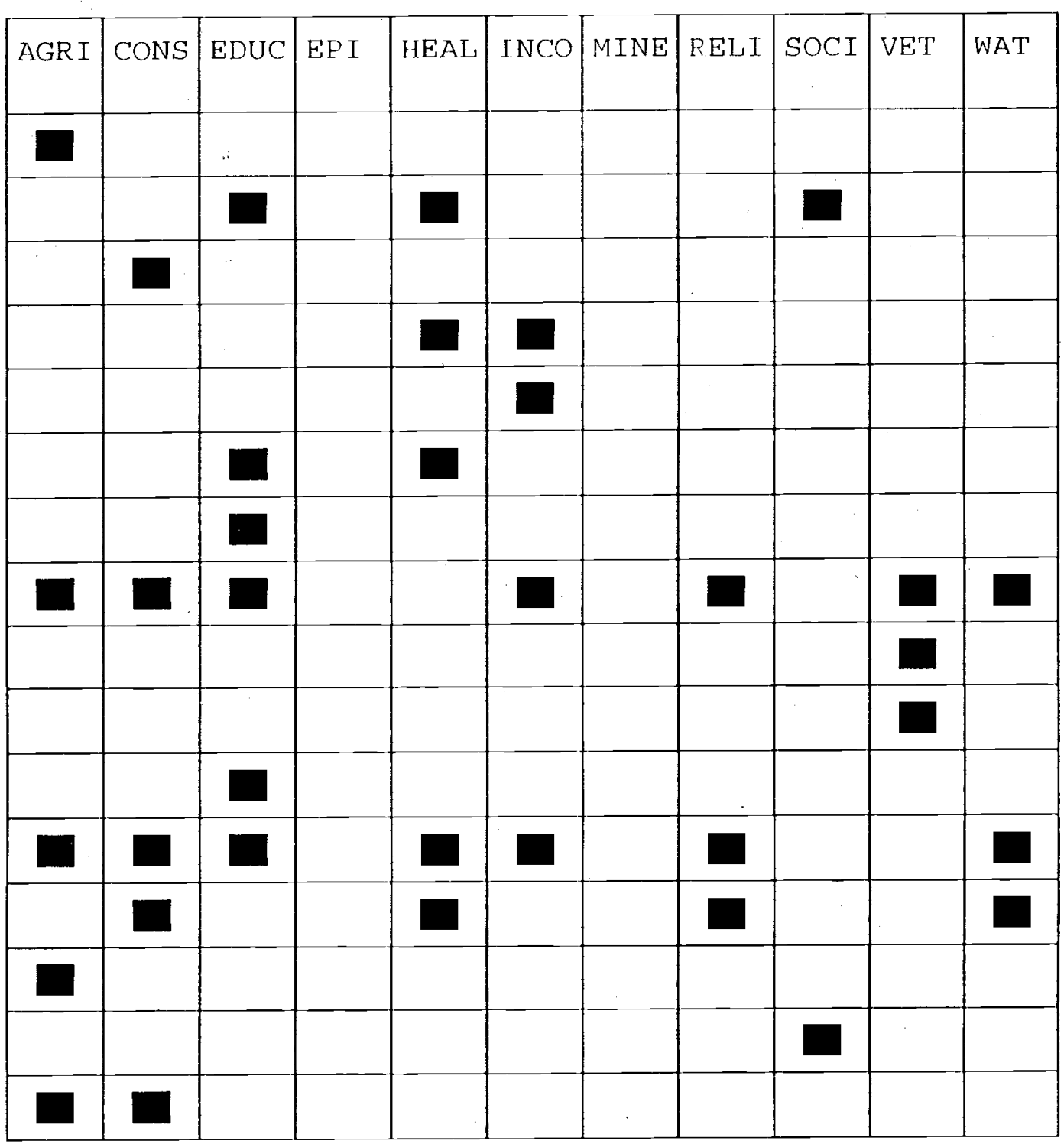

\begin{tabular}{|lllll|}
\hline $\mathrm{T}$ & $\mathrm{O}$ & $\mathrm{T}$ & $\mathrm{A}$ & $\mathrm{L}$ \\
\hline
\end{tabular}

\begin{tabular}{|l|l|l|l|l|l|l|l|l|l|l|}
\hline 85 & 98 & 69 & 13 & 89 & 59 & 6 & 32 & 29 & 15 & 87 \\
\hline
\end{tabular}

OPERATION SITES OF NGOs During 1995

\begin{tabular}{||l|c|}
\hline NUMBER OF NGOS WORKING FOR REFUGEES & 25 \\
\hline \hline NUMBER OF NGOS WORKING CROSS-BORDER & 144 \\
\hline NUMBER OF NGOS WORKING FOR REFUGEES \& CROSS-BORDER & 74 \\
\hline \hline TO TA $L:$ & 243 \\
\hline
\end{tabular}




\section{AFFILIATION OF NGOS}

\section{AGENCIES}

A-AID

$A A A$

AABRAR

AACRP

AAEA

AAID

$A C A$

ACAA

ACDA

ACDO

ACLU

ACRD

ACRO

ADA

ADAg

ADARA

ADO

ADP

ADS

AERRA

AFRANE

AFRC

AFRO

AFS

AG-TTP

AHDS

AHSAO

AIC

AICC

AICF

AINA

AITM

AMA

AM I - A

AMRAN

AMRC

AMWS

ANAR

ANH

AOGH

APA

APUA

APWO

ARA

ARAD

ARADA

ARC

ARCAR

ARCC

ARCON

ARCS
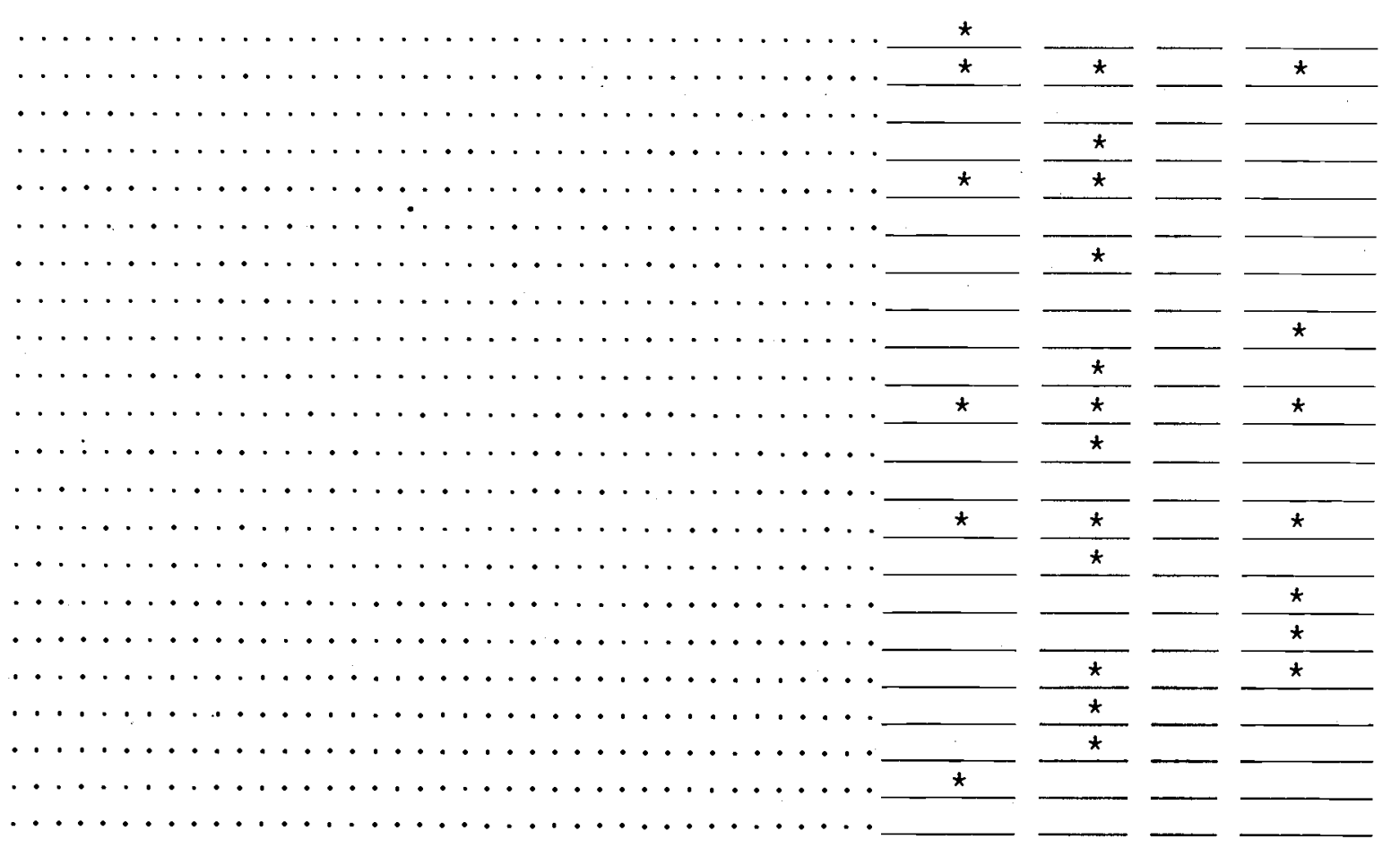

$\ldots \ldots \ldots \ldots \ldots \ldots \ldots \ldots \ldots \ldots \ldots \ldots$
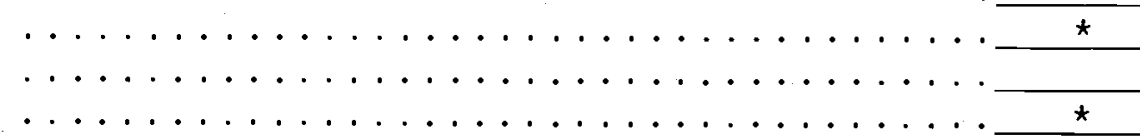

$\star$

$\frac{\star \star}{\star}$

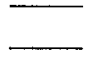

$$
-
$$
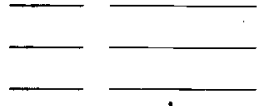

$\frac{\hbar}{\star \star}$

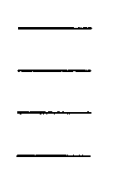

$+$

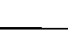
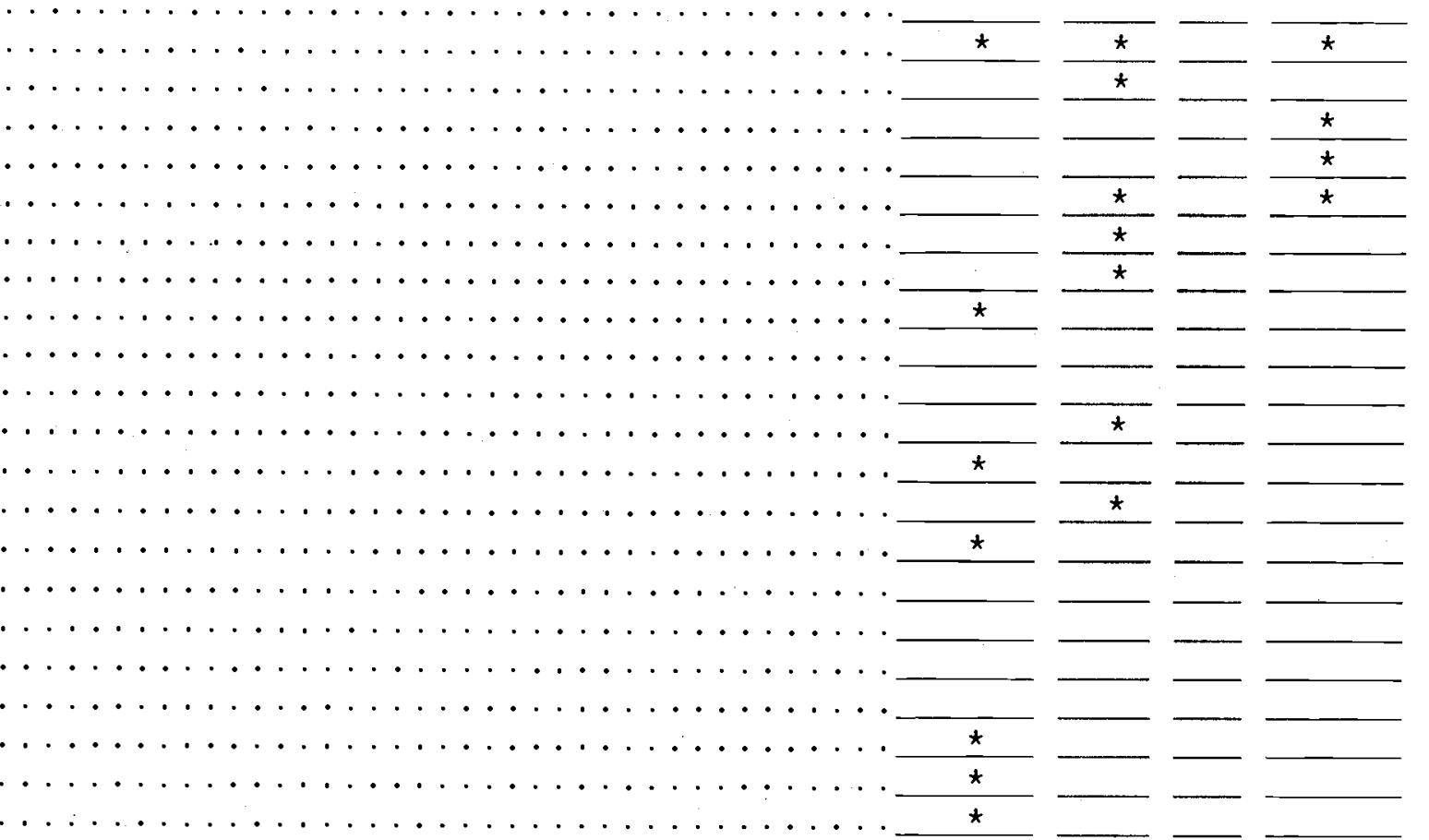

$\ldots \ldots \ldots \ldots \ldots \ldots \ldots \ldots \ldots$

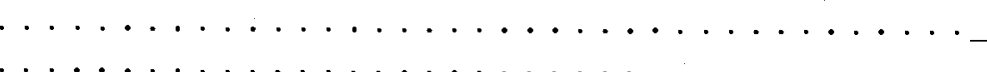

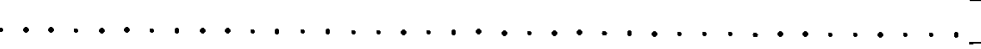

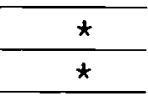

$\frac{\star}{\star}$
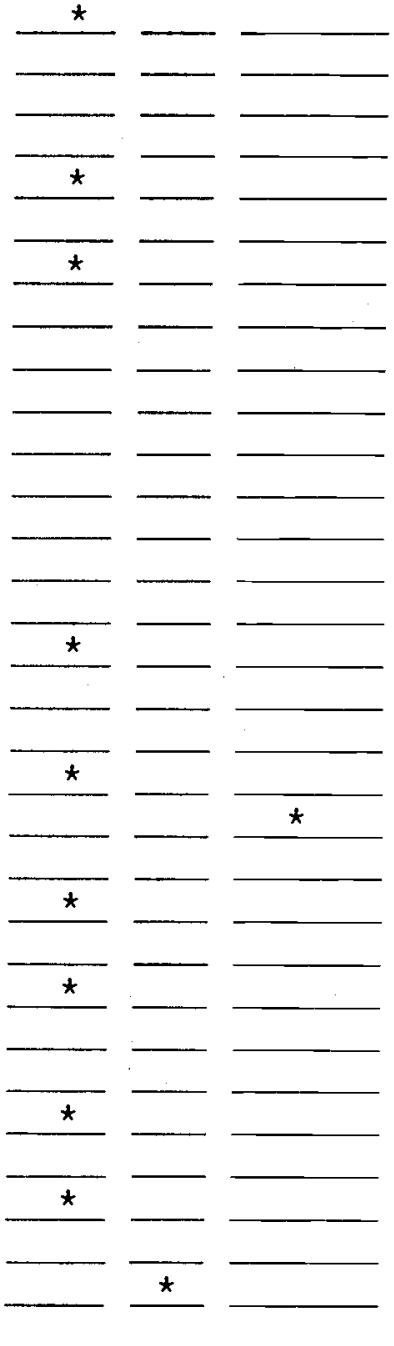
ARDA

ARDCD

ARDO

ARDP

AREA-1

AREA-2

ARF

ARIA

ARLO

ARM

ARO

ARPD

ARR

ARRAO

ART

ASDO

ATA/AP

ATC

AURC

AVICEN

AWC

AWEC

AWRC

AWWD

AWWO

BCURA

BDA

BEFARe

BONIAD

BRC

BURC

CARE

CARP

CAWC

CBR

CCA

CHA

CHTG

COAR

CRAA

CRFA

DACAAR

DARA

DCA

DgCAR

DS

EAFA

ECAR

EDS

EMAR

ERU

ESAR

FRF

GAA
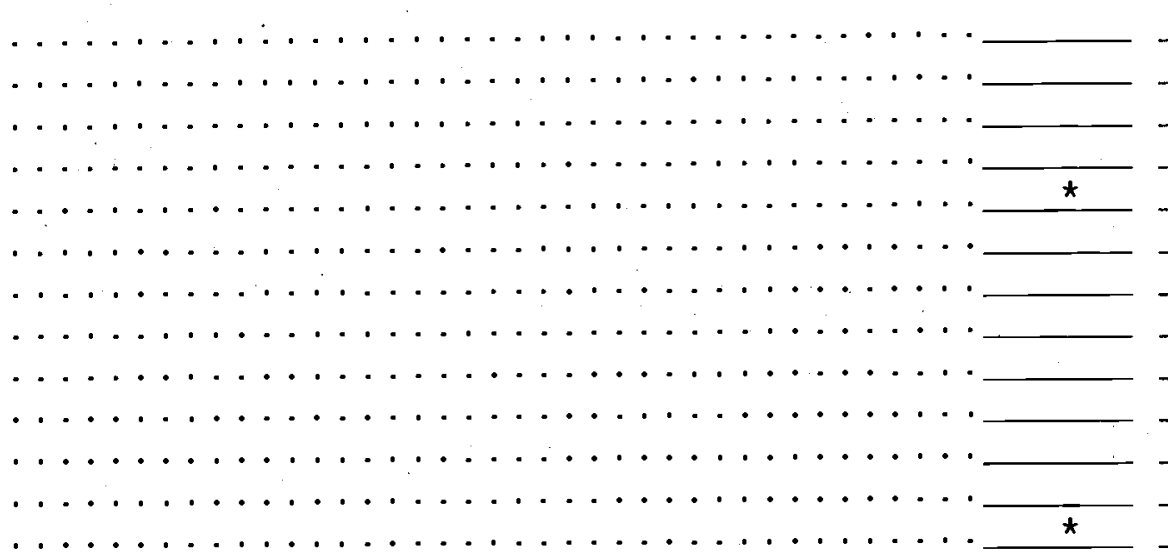

$\ldots \ldots \ldots \ldots \ldots \ldots \ldots \ldots \ldots \ldots \ldots \ldots \ldots$
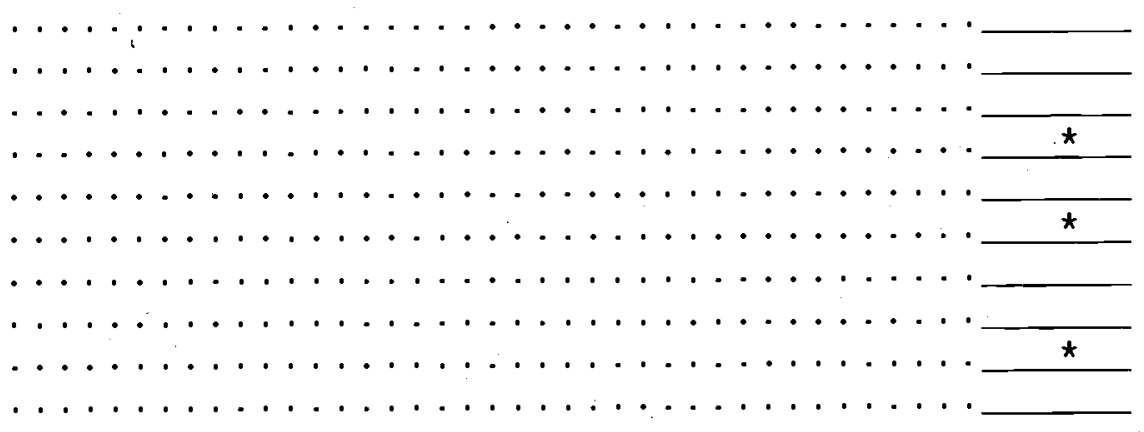$$
\text { . }
$$

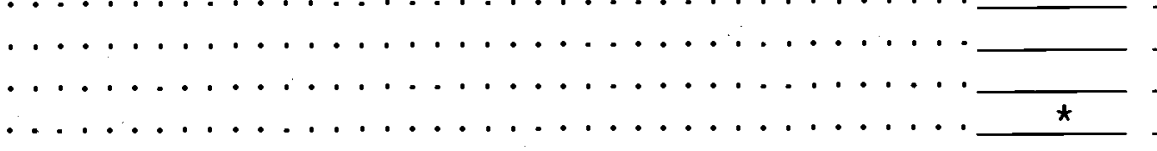

$$
\text { . }
$$

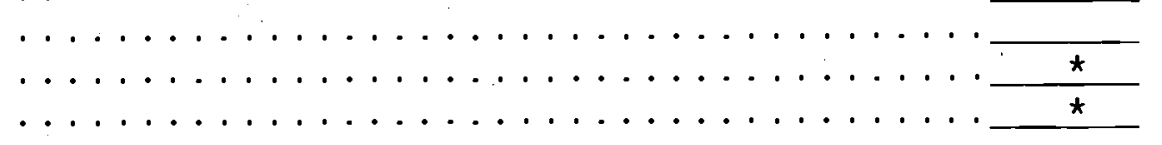$$
\ldots
$$
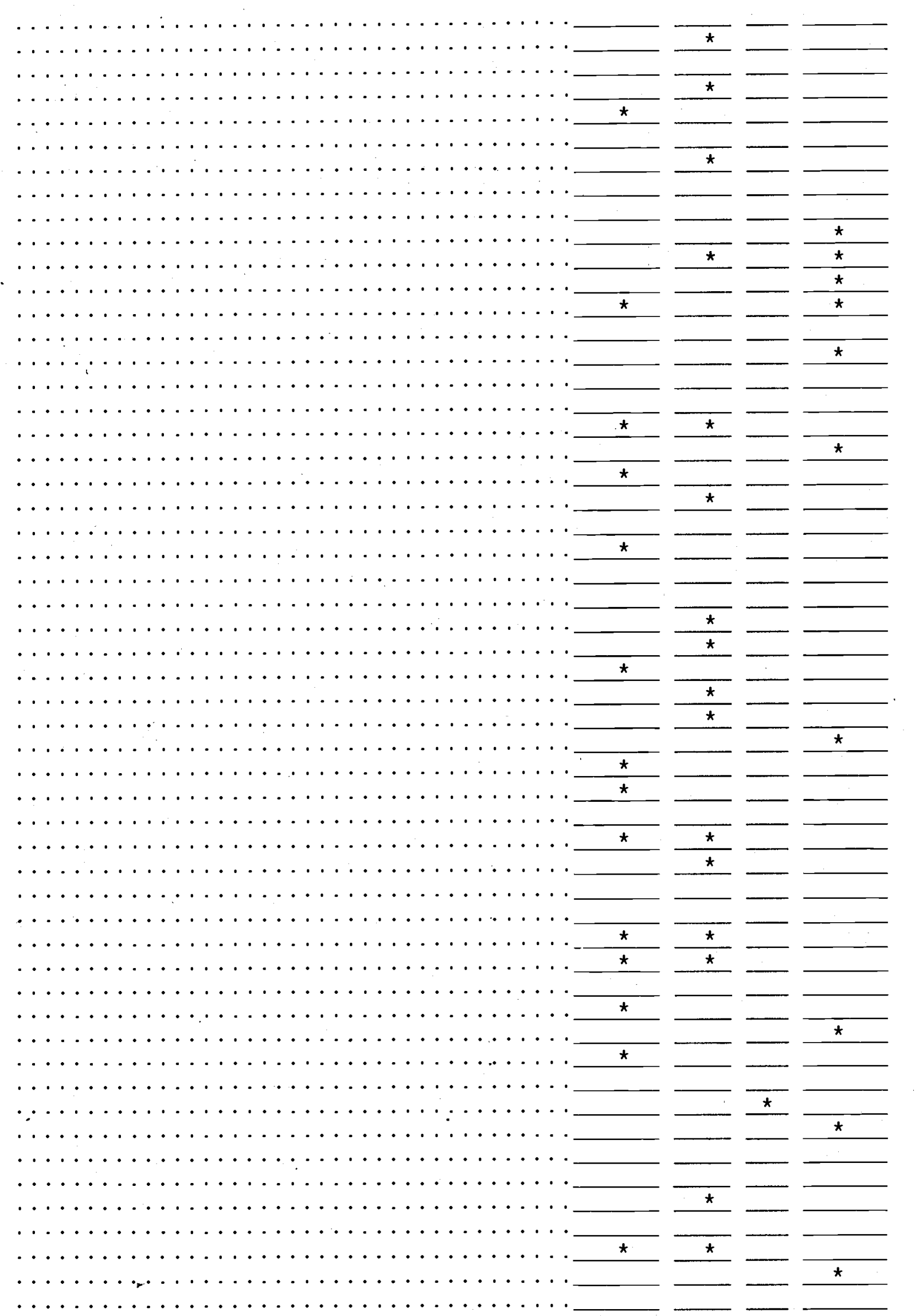

$*$

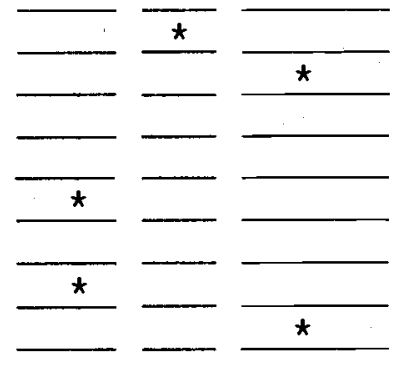


GAF

GP - UK

GRC

GRSP

$\mathrm{HAF}$

HAFO

$\mathrm{HCI}$

HDCAW

HELP

HERI

$\mathrm{HI}$

HRAA

HRCA

HRP

$\mathrm{HT}$

HWRA

IAAAE

IAHC

IAM

IAU

ICD

ICRC

IFRCS

IHSAN

I IRO

IMC

IOC

IOM

IRC

IRC/RPA

ISRA

JAMS

JCE

JIFF

JWMM

KMA

KNF

KPRO

KRCS

KWA

LB I

I.D I

LKRO

IQO

LRO

MADERA

MAI

MARUF

MAWA

MCI

MCPA

$M D C$

IDM

IERLIN
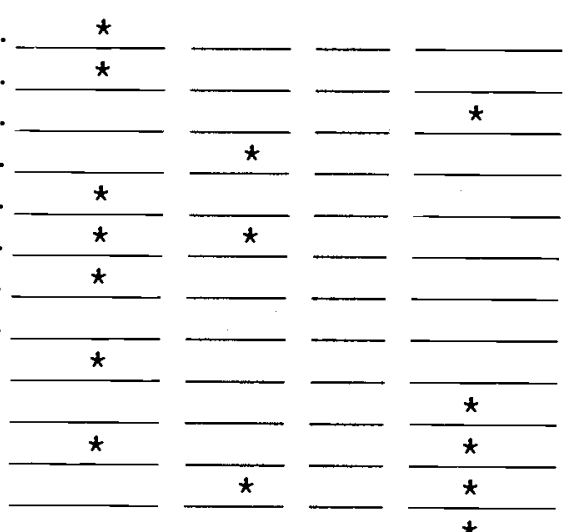

$\star$
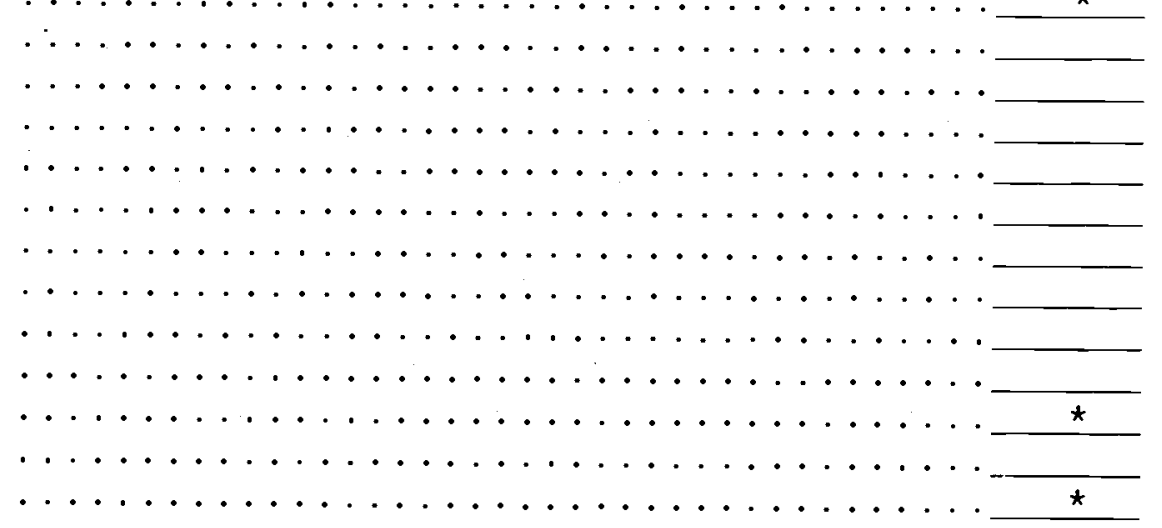

*

*

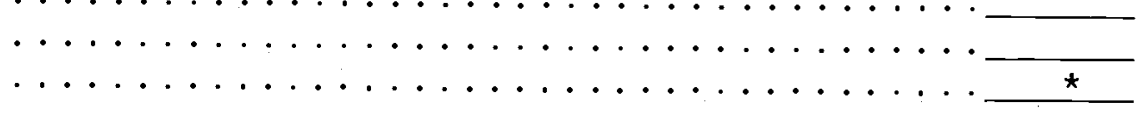

$$
\text { . }
$$$$
\text { . }
$$

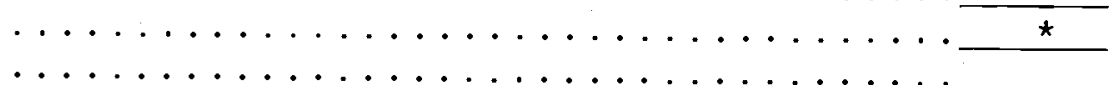$$
\text { . }
$$

$$
\begin{aligned}
& . \\
& . \\
& . \\
& . \\
& . \\
& . \\
& . \\
& . \\
& . \\
& . \\
& . \\
& .
\end{aligned}
$$


MF

MHC

MMC

MPO

MRC

MRCA

MRORA

MSF

MSOA

NAC

NADA

NCRA

NERO

NERU

NPO/RRAA

NRO

OHA

OMAID

OMAR

ORA

OV

OXFAM

PARDC

PDA

PETRA

PRB

PRCS

PRS

PSD

PSF

RADA

RAFA

RAH

RBS

RDA

RDM

RDP

RDW

ROAOW

RPA

RPSA

RSSA

SAA

SADA

SAMSOR

SAVE

SC-US

SCA

SCF - UK

SERVE

SGAA

SGRO

SHACA

SJAWO
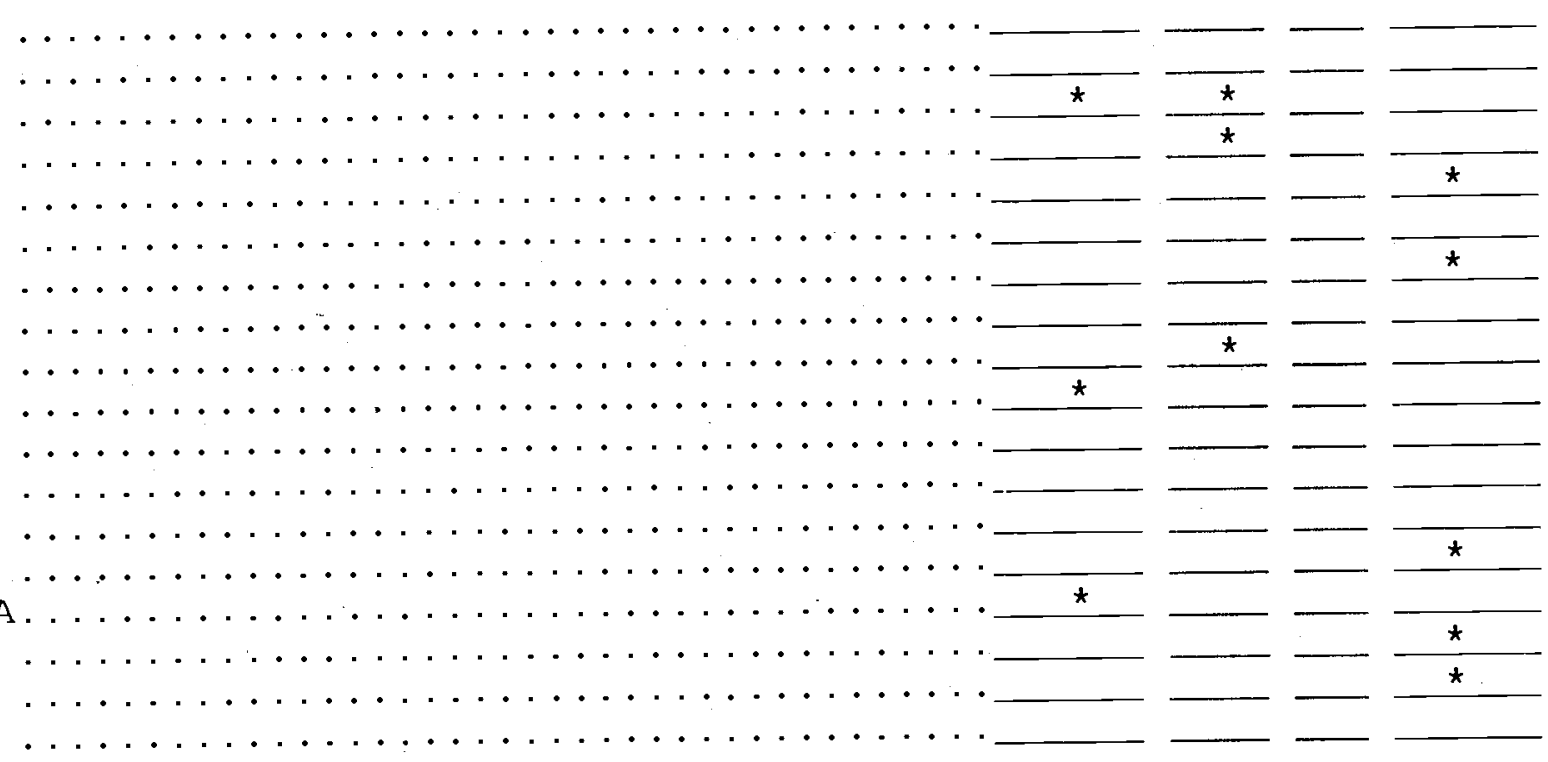$$
\text { . }
$$
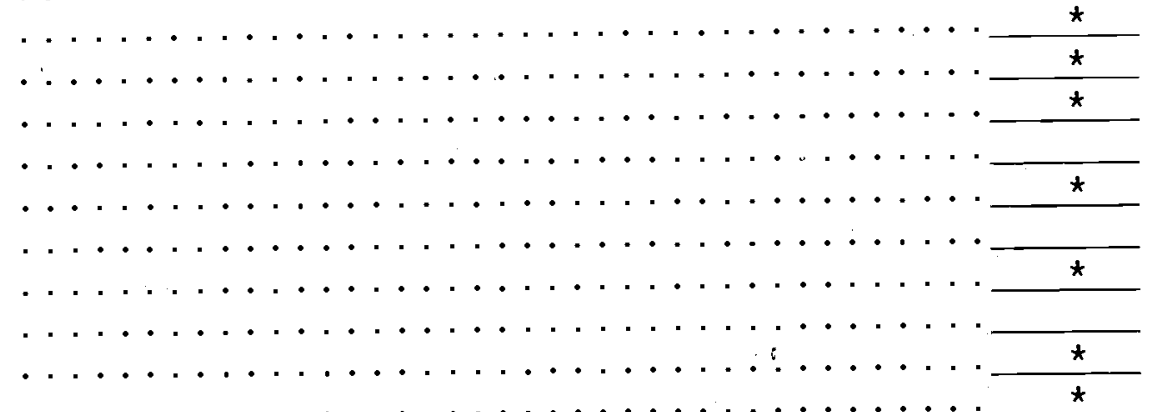

-
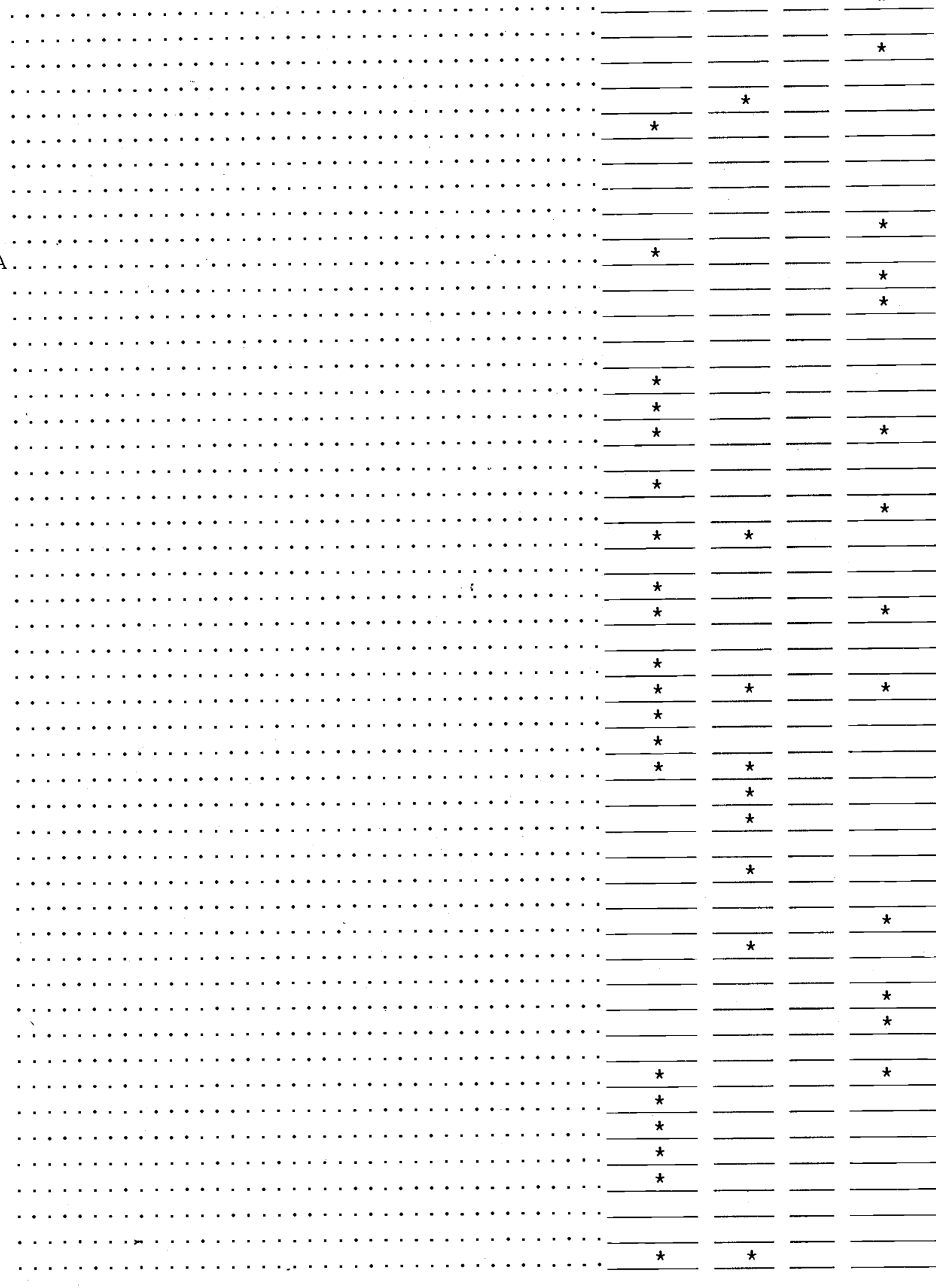
SNI

SO

SOLID

SOS-PG

SRA

SRCDA

SRCS

SRDA

SRO

SRRC

START

SWAARR

SWARO

TAMIR

TDH

TRO

UAAR

UAE - RCS

UMCA

UNO/EPA

VARA

VSO

WFA

WRA

WRC

WRF

WROR

WUFA

YARA

TOTAL
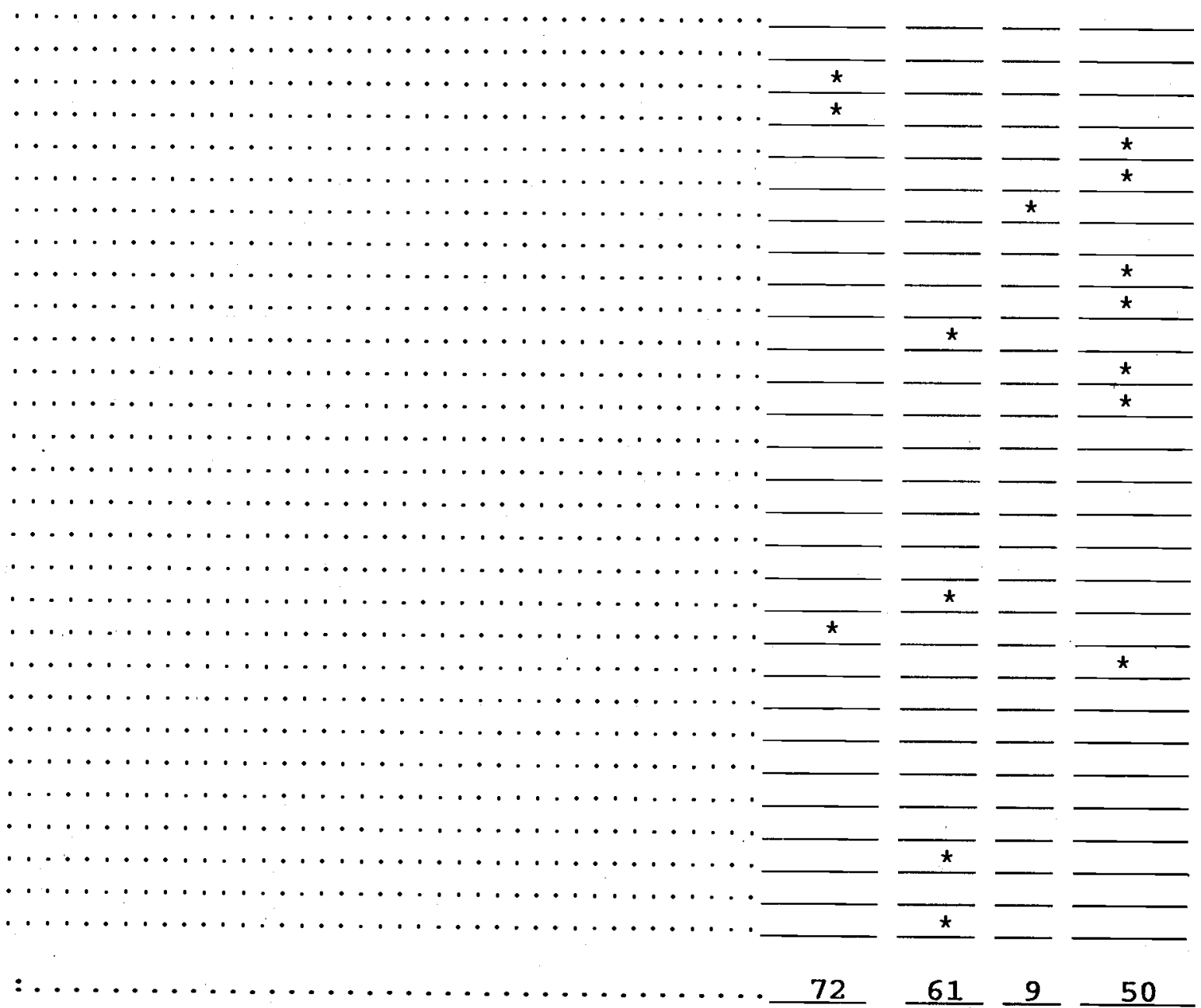

\begin{tabular}{|l|l|}
\hline ACBAR members : & 72 \\
\hline ANCB members : & 61 (including 21 ACBAR members) \\
\hline ICC members : & 9 (including 2 ACBAR members) \\
\hline SWABAC members: & 50 (including 13 ACBAR members) \\
\hline Other NGOs : & 89 \\
\hline \hline T O T A L : & $242^{1}$ \\
\hline
\end{tabular}

Note : NCA/NRC is a member agency of ACBAR. It is a funding agency and does not implement any projects.

${ }^{1} \mathrm{ADA}, \dot{A} \mathrm{CLU}$ and MCPA are members of ACBAR, ANCB and SWABAC. 
Funding includes cash donations and donations in kind such as agricultural inputs .

\begin{tabular}{|c|c|}
\hline \multirow[t]{2}{*}{$\mathbf{A A}$} & 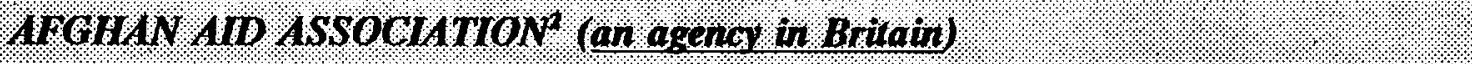 \\
\hline & ARDA \\
\hline \multirow[t]{2}{*}{ 100. } & ASSOCHMTON FOR DEIETOPMENT AWD COOPERATHON. \\
\hline & $\mathrm{ARC}$ \\
\hline \multirow{2}{*}{ 119. } & 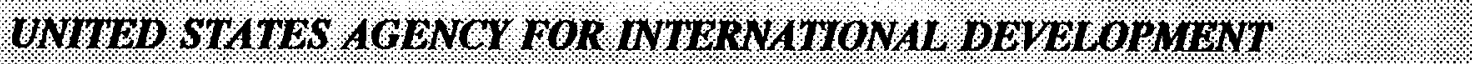 \\
\hline & CoAR, DACAAR, III, IRC \\
\hline \multirow[t]{2}{*}{ arcon } & 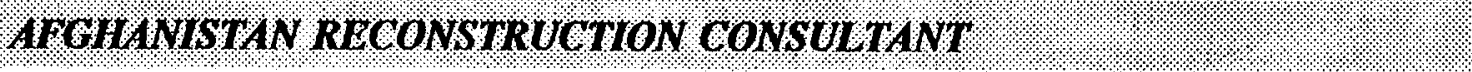 \\
\hline & $A R I A, \quad P R B$ \\
\hline \multirow[t]{2}{*}{$151 \% 10 \%$} & 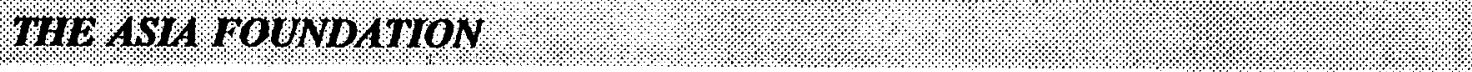 \\
\hline & ADS, WUFA \\
\hline \multirow[t]{2}{*}{ 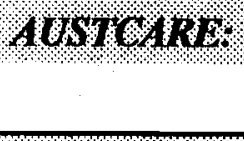 } & $195 \% 180$ \\
\hline & ECAR, SC-US \\
\hline \multirow[t]{2}{*}{$\mathrm{BPRH}$} & BURE IO OF POPULATION, REFUGEES, AND MTGRATION \\
\hline & IRC, SU-US \\
\hline \multirow[t]{2}{*}{$0 B H \%$} & GWHSTOFFET BI IND MISSTON \\
\hline & SERVE \\
\hline \multirow[t]{2}{*}{ orr. } & CANGOGW WTERNATTONAI DEVELOPMENT AGGNCY \\
\hline & $\begin{array}{l}\text { AACRP, ACDA, ADAg, ADARA, AIISAO, AITM, AMRAN, ARO, AURC, AWEC, AWEC, } \\
\text { AWRC, AWWD, BDA, BRC, BURC, CCA, CRFA, EMAR, IAFO, ICI, HDCAW, IRC, } \\
\text { RWA, MARUF, MAWA, MRORA, PDA, PRB, PRS, RDA, ROAOW, SO, SRCDA, } \\
\text { STARTY, }\end{array}$ \\
\hline \multirow[t]{2}{*}{ OOMH } & 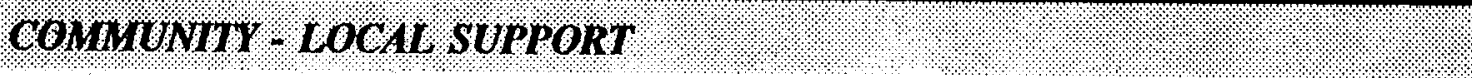 \\
\hline & ARLO, VARA， WRA \\
\hline $2010 \%$ & 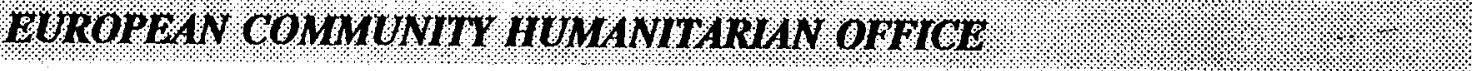 \\
\hline & AMI-A， DACAAR， GAA， IMC， MDM， MERLIN, MSF， PSF \\
\hline
\end{tabular}

2. It should be noticed that there is an Afghan NGQ with the same name which is an implementing agency, not a donor. 


\begin{tabular}{|c|c|}
\hline \multirow[t]{2}{*}{ EU: } & EUROTEAN UNION \\
\hline & $\begin{array}{l}\text { A-AID, AABRAR, ADA, AFRANE, AG-TTP, AHDS, AMI-A, ARCS, } \\
\text { AREA-1, ATC, AVICEN, CARE, CHA, COAR, DACAAR, HELP, HT, } \\
\text { IFRCS, IMC, IRC, MADERA, MCPA, MDM, MSF, OMAR, ORA, OV, } \\
\text { OXFAM, RPA, SCA, SGAA, SJAWO, SOLID, SOS-PG }\end{array}$ \\
\hline \multirow[t]{2}{*}{ FAO } & FOOD ANB AGRICULTURE ORCANIZAITION \\
\hline & $\begin{array}{l}\text { AAA, AAEA, ACA, ACDO, ACRD, ACRO, ADA, ADAg, AFRANE, AFRO, } \\
\text { AFS, AHSAO, AINA, AMRAN, ANH, APWO, ARADA, ARDA, ARDO, } \\
\text { ARDP, ARF, ARIA, ARO, ARR, AURC, BCURA, BURC, COAR, } \\
\text { DACAAR, DCA, EDS, FRF, GAF, GRSP, HAFO, HERI, HRP, IHSAN, } \\
\text { IRC, ISRA, JCE, KNF, LBI, LRO, MADERA, MAWA, MCI, MRORA, } \\
\text { NAC, NCRA, NERU, NPO/RRAA, PETRA, PRB, PRS, RAFA, RAH, } \\
\text { RDA, RDM, RDW, SCA, SERVE, SJAWO, SOS-PG, SOLID, START, } \\
\text { VARA, WRC, WROR, YARA }\end{array}$ \\
\hline \multirow[t]{2}{*}{ GT2: } & GERMIN AGENCY FOR TECHNICAL COOPERATION \\
\hline & AG-TTP, ARC, AREA-1, ARO, BEFARe, RDM, RPSA, START \\
\hline \multirow[t]{2}{*}{ HAF } & HEIP TWE ARGHANS FOUNDATION \\
\hline & MRCA， RADA \\
\hline \multirow[t]{2}{*}{ TORC: } & WTERNATIONAL COMMTTIEE OF THE RED CROSS \\
\hline & ARCS, MMC， PRCS， SGRO， SRDA \\
\hline \multirow[t]{2}{*}{ MRo: } & SY AMIC INTERNATIONAL RELIEF ORGANIRATION \\
\hline & ARLO \\
\hline \multirow[t]{2}{*}{ IRC: } & MTERNATIONAL RESCUE COMMMTTEE-RURAL ASSISTANCE PROGRM \\
\hline & $\begin{array}{l}\text { AABRAR, ACRD, AITM, ARC, ARDCD, ARO, AWWO, CHA, HAFO, } \\
\text { JIFF, NPO/RRAA, OMIAD, PSD, SJAWO, UMCA }\end{array}$ \\
\hline \multirow[t]{2}{*}{ RUPFA: } & 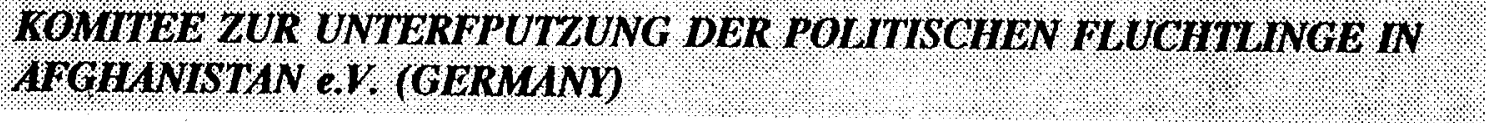 \\
\hline & ARF \\
\hline \multirow[t]{2}{*}{ NAC: } & NORWEGIAN AFGHUNISTAN COMMITIEE \\
\hline & HAFO, MSOA, SOLID, UMCA \\
\hline \multirow[t]{2}{*}{ NoniB: } & NETHERLANDS ORGANIZATION FOR INTL DEVELOPMENT COOPERATION \\
\hline & ARC, CCA, CHA, GRSP, IAHC, OMAR, PSD \\
\hline \multirow[t]{2}{*}{ NCAINRE: } & NORWEGUN CHURCH ADINORWEGUN REFUGEE COUNCH. \\
\hline & $\begin{array}{l}\text { ADA, AICF, ATA/AP, CAWC, COAR, GRSP, IAM, IRC, NPO/RRAA, } \\
\text { ORA, PSD }\end{array}$ \\
\hline
\end{tabular}




\begin{tabular}{|c|c|}
\hline \multirow[t]{2}{*}{ OOA. } & OVERSE AS DEVELOPMIENT IGRNCY \\
\hline & A-AID, CARE, HT, MERLIN, OV, SGAA，VSO \\
\hline \multirow[t]{2}{*}{$0 \mathrm{WHN}$} & $0 \times 191 \%$ \\
\hline & CCA， DACAAR， IAHC， MADERA \\
\hline \multirow[t]{2}{*}{ PVTr } & PRV 1 TO OONORS \\
\hline & $\begin{array}{l}\text { AFRANE, AFRC, AHSAO, AICC, AMRAN, AMWS, ARCON, AREA-1, } \\
\text { ARLO, AWRC, CAWC, CCA, DS, ESAR, IAU, IMC, MPO, MRCA, PRB, } \\
\text { RADA, SHACA, SO, SOLID, WRC }\end{array}$ \\
\hline \multirow[t]{2}{*}{$\mathrm{SOA}$} & 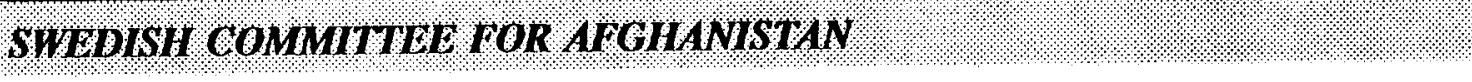 \\
\hline & AREA-1， HWRA， SOLID \\
\hline \multirow[t]{2}{*}{ SV. } & 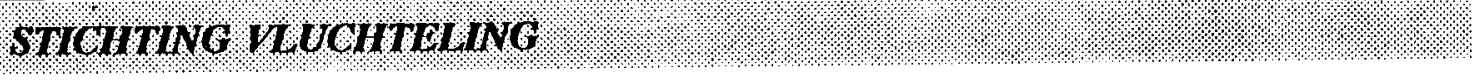 \\
\hline & AREA-1，ARR， COAR， IRC \\
\hline \multirow[t]{2}{*}{$\operatorname{TaAR} /$} & TEAR RONO \\
\hline & ORA， SERVE \\
\hline \multirow[t]{2}{*}{ UNCH. } & 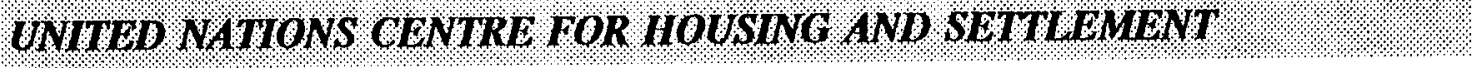 \\
\hline & AMRAN, COAR, ESAR, SNI \\
\hline \multirow[t]{2}{*}{ UHOP. } & UNTTED NATONS DEVELOPMENT PROGRAMME. \\
\hline & $\begin{array}{l}\text { ADA, AFRANE, ARDA, ARCAR, AURC, BURC, CBR, CHA, DACAAR, } \\
\text { GRSP, HAFO, HERI, HRAA, IAM, KMA, LKRO, MARUF, MRORA, ORA, } \\
\text { PSD, RAFA， SERVE， SGAA， SJAWO, START, SWAARR， SWARO，WFA， } \\
\text { YARA }\end{array}$ \\
\hline \multirow[t]{2}{*}{ UNDOP } & UNHEO NATIONS DRUG CONTROL PROGRAMME. \\
\hline & $\begin{array}{l}\text { A-AID, ADA, ANAR, ARCAR, AWC, ESAR, HAFO, IHSAN, ISRA, } \\
\text { KNF, MADERA, MCI, MRORA, NERU, PRB, SERVE }\end{array}$ \\
\hline \multirow[t]{2}{*}{ UNIFSCO } & UNMTD NATIONS GDUCATTONAL, SCIRNTHIC \& GULTURAL ORGAN. \\
\hline & ASDO, CRFA, MRORA \\
\hline \multirow[t]{2}{*}{ UNHCR: } & UNMED NATIONS HIGH COMMISSTON FOR REFUGLES \\
\hline & $\begin{array}{l}\text { AAA, AACRP, ACDO, AERRA, AFRANE, AINA, AMRAN, ANH, ARADA, } \\
\text { ARDA, ARDO, AREA-1, ARM, AURC, BCURA, BDA, BURC, CCA, CHA, } \\
\text { CHTG, DACAAR, DARA, EAFA, EMAR, GRC, HAF, HI, HRCA, IAM, } \\
\text { IHSAN, IRC, KMA, KNF, KRCS, LKRO, MCI, MDM, MRC, MRORA, } \\
\text { NAC, NERU, NPO/RRAA, NRO, OV, PETRA, PSD, RAH, RDA, RSSA, } \\
\text { SAMSOR, SAVE, SC-US, SCA, SERVE, SGRO, SJAWO, SNI, SO, } \\
\text { SOLID, SRO, SRRC, SWARO, VARA, WRF, YARA }\end{array}$ \\
\hline
\end{tabular}




\begin{tabular}{|c|c|}
\hline \multirow[t]{2}{*}{ WNOS } & 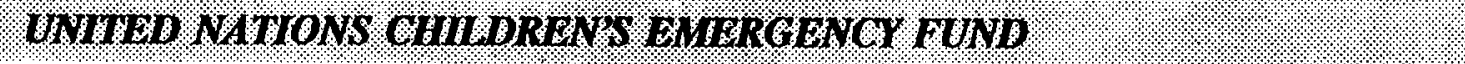 \\
\hline & $\begin{array}{l}\text { AHDS, ARPD, CCA, EDS, GAF, IRC, ISRA, KPRO, MADERA, MMC, } \\
\text { NAC, RAFA, SO }\end{array}$ \\
\hline \multirow[t]{2}{*}{$6 n O 01 \%$. } & 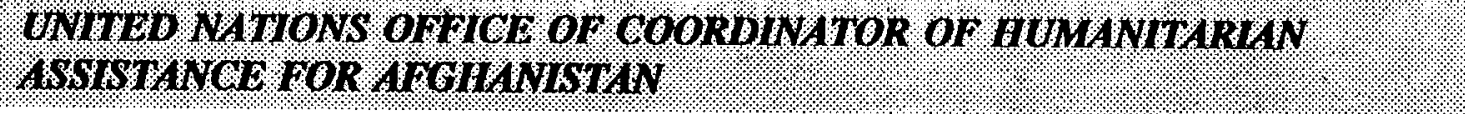 \\
\hline & ATC, MCPA， MDC， OMAR \\
\hline \multirow[t]{2}{*}{ Mir. } & 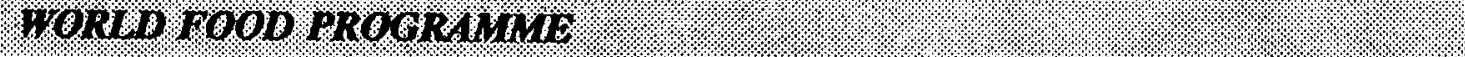 \\
\hline & $\begin{array}{l}\text { A-AID, AAA, AACRP, AAEA, AAID, ACAA, ACLU, ADAg, ADO, } \\
\text { AERRA, AFRC, AFRO, AFS, AMRAN, APWO, ARADA, ARC, ARCAR, } \\
\text { ARCC, ARDA, ARDO, AREA-2, ARM, ARO, ARPD, ARR, ARRAO, } \\
\text { AURC, AWWO, BCURA, BDA, BURC, CARE, CCA, DARA, EAFA, EMAR, } \\
\text { ERU, ESAR, FRF, GAA, GRC, HCI, HERI, HRAA, HRCA, IHSAN, } \\
\text { KMA, KNF, LKRO, MARUF, MCI, MDM, MRC, MRORA, MSOA, NAC, } \\
\text { NCRA, NERO, NERU, NPO/RRAA, NRO, OHA, PDA, PETRA, PRB, } \\
\text { PRS, PSD, RAFA, RAH, RDA, RSSA, SAMSOR, SAVE, SERVE, SGRO, } \\
\text { SJAWO, SO, SRA, SRCDA, SRDA, SRO, SRRC, START, SWAARR, } \\
\text { SWARO, TRO, VARA, WRC, WRF }\end{array}$ \\
\hline \multirow[t]{2}{*}{110} & 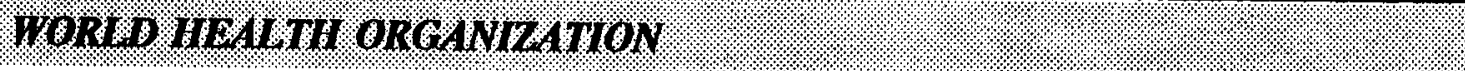 \\
\hline & AREA-2， CBR， MMC， SGAA，UMCA \\
\hline \multirow[t]{2}{*}{ W0108\% } & 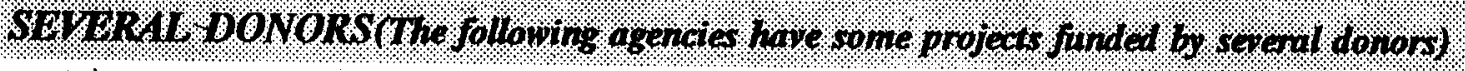 \\
\hline & $\begin{array}{l}\text { AABRAR; AFRANE, AOGH, ARC, ARCS, ARDA, ARF, BURC, CARP, } \\
\text { DACAAR, DS, ERU, I IRO, JWMM, KPRO, MARUF, MHC, MSF, NAC, } \\
\text { NPO/RRAA, ORA, OXFAM, RADA, SAVE, SERVE, SO, UAAR }\end{array}$ \\
\hline
\end{tabular}

NOTE : Like other information on the projects in the Database, the information on funding source(s) has been provided by the implementing agencies. If, in the column of "FUNDED BY", the name of the implementing agency or the word "Self" is written, it means that the funding for that project has come from the agency itself. "*'Donors" means that the project is funded by several donors. In a few places, the full abbreviation of donor agencies has not been written because of lack of space in the column, e.g. UNES for UNESCO and OCHA for UNOCHA.

Most of international NGOs also receive funds from their own governments e.g., DACAAR from Danish Government and AVICEN from French Government. 


\section{AGENCY COUNTRY OF ORIGIN}

\section{AFGHANISTAN}

\begin{tabular}{|c|c|}
\hline AAA & $\begin{array}{l}\text { AFGHAN AID ASSOCIATION } \\
\text { AFGHANISTAN AGRICULTURE\&CONSTRUCTION REHABILITATION PROJECT }\end{array}$ \\
\hline AACRP & $\begin{array}{l}\text { AFGHANISTAN AGRICULTURE\&CONSTRUCTION REHAB ILITATION PRUUECI } \\
\text { AFGHAN AGRICULTURAL \& ENGINEERING AGENCY }\end{array}$ \\
\hline AAEA & \\
\hline AAID & \\
\hline $\begin{array}{l}\text { ACA } \\
\text { ACAA }\end{array}$ & $\begin{array}{l}\text { ABADY CONSTRUCTION AGENCY } \\
\text { ASMAIY CONSTRUCTION \& AGRICULTURAL AGENCY }\end{array}$ \\
\hline $\begin{array}{l}A C A A \\
A C D A\end{array}$ & $\begin{array}{l}\text { ASMAIY CONSTRUCTION \& AGRICULTURAL AGENCY } \\
\text { AFGHAN COMMUNITY DEVELOPMENT AGENCY }\end{array}$ \\
\hline $\begin{array}{l}A C D A \\
A C D O\end{array}$ & $\begin{array}{l}\text { AFGHAN COMMUNITY DEVELOPMENT AGENCY } \\
\text { AFGHAN COMMUNITY DEVELOPMENT ORGANIZATION }\end{array}$ \\
\hline $\mathrm{ACl}\lrcorner \mathrm{U}$ & $\begin{array}{l}\text { AFGHAN COMMUNITY DEVELOPMENT ORGANIZATION } \\
\text { AFGHAN CONSTRUCTION \& LOGISTICS UNIT }\end{array}$ \\
\hline ACRD & $\begin{array}{l}\text { AFGHAN CONSTRUCTION \& LOGISTICS UNIT } \\
\text { AFGHAN CENTER FOR RURAL DEVELOPMENT }\end{array}$ \\
\hline ACRO & $\begin{array}{ll}\text { AFGHAN } & \text { CENTER FOR RURAL DEVELOPMENT } \\
\text { AFGHAN REHABILITATION \& CONSTRUCTION ORGANIZATION }\end{array}$ \\
\hline $\mathrm{ADA}$ & $\begin{array}{ll}\text { AFGHAN REHABILITATION \& CONSTRUCTION ORGANIZATION } \\
\text { AFGHAN DEVELOPMENT ASSOCIATION }\end{array}$ \\
\hline ADAg & $\begin{array}{ll}\text { AFGHAN } & \text { DEVELOPMENT ASSOCIATION } \\
\text { AFGHAN DEVELOPMENT AGENCY } & \end{array}$ \\
\hline ADARA & $\begin{array}{l}\text { AFGHAN DEVELOPMENT AGENCY } \\
\text { AFGHANISTAN DEVELOPMENT AND RECONSTRUCTION ASSOCIATION }\end{array}$ \\
\hline ADO & AFGHANISTAN DEVELUPIENT ORGANIZATION \\
\hline ADP & AFGHAN DEVELOPMENT PROGRAM \\
\hline $\mathrm{ADS}$ & AFGHAN DISABLED SOCIETY \\
\hline AERRA & ASSOCIATION OF EMERGENCY RELIEF \& RECONS. IN AFGHANISTAN \\
\hline$A F^{\prime} R C$ & AFGHANISTAN RECONSTRUCTION COUNCIL \\
\hline AFRO & AFGHAN FARMERS RELIEF ORGANIZATION \\
\hline AFS & AGENCY FOR FARMING SUPPORT \\
\hline AHDS & AFGHAN HEALTH \& DEVELOPMENT SERVICES \\
\hline AHSAO & AFGHANS HEALTH \& SOCIAL ASSISTANCE ORGANIZATION \\
\hline $\mathrm{AIC}$ & AFGHAN INFORMATION CENTER \\
\hline AICC & AL-DAWA ISLAMIC CULTURAL CENTER \\
\hline AINA & AFGHAN ISLAMIC NATIONAL AGENCY \\
\hline AITM & AFGHAN INKISHAFEE TARBIAWEE MARKAZ \\
\hline AMA & AFGHAN MEDICAL AID \\
\hline AMRAN & AFGHAN MOBILE RECONSTRUCTION ASSOCIATION \\
\hline AMRC & AFGHAN MEDIA RESOURCE CENTER \\
\hline AMWS & AFGHAN MUSLIM WOMEN SOCIETY \\
\hline ANAR & AFGHANISTAN NATIONAL ASSOCIATION FOR REHABILITATION \\
\hline AOGH & AFGHAN OBSTETRICS/GYNECOLOGY HOSPITAL \\
\hline AFA & AFGHAN PLANNING AGENCY \\
\hline APUA & ASSOCIATION OF PROFESSORS OF THE UNIVERSITIES OF AFGHANISTAN \\
\hline APWO & AFGHAN PUBLIC WELFARE ORGANIZATION \\
\hline ARA & AGR I CULTURE REHABILITATION OF AFGHANISTAN \\
\hline ARAD & AFGHANISTAN RECONSTRUCTION AUTHORITY DEVELOPMEN'T \\
\hline ARADA & AGENCY FOR REHABILITATION ASSISTANCE \& DEVELOP. OF AFGHANISTAN \\
\hline ARCAR & ARIANA REHABILITATION COMMITTEE FOR AFGHANISTAN RECONSTRUCTION \\
\hline ARCC & AFGHANISTAN RECONSTRUCTION CONSULTATION COMMITTEE \\
\hline ARCS & AFGHAN RED CRESCENT SOCIETY \\
\hline ARDA & AGENCY FOR RURAL DEVELOPMENT OF AFGHANISTAN \\
\hline ARDCD & AFGHAN RECONSTRUCTION \& DRUG CONTROL DEPARTMENT \\
\hline ARDO & AFGHANISTAN REHABILITATION \& DEVELOPMENT ORGANIZATION \\
\hline ARDF & AFGHANISTAN REHABILITATION \& DEVEI_OPMENT PROGRAM \\
\hline & AGENCY FOR REHABILITATION \& ENERGY CONSERVATION IN AFGHANISTAN \\
\hline REA - 2 & AFGHANISTAN RECONSTRUCTION ASSOCIATION \\
\hline
\end{tabular}




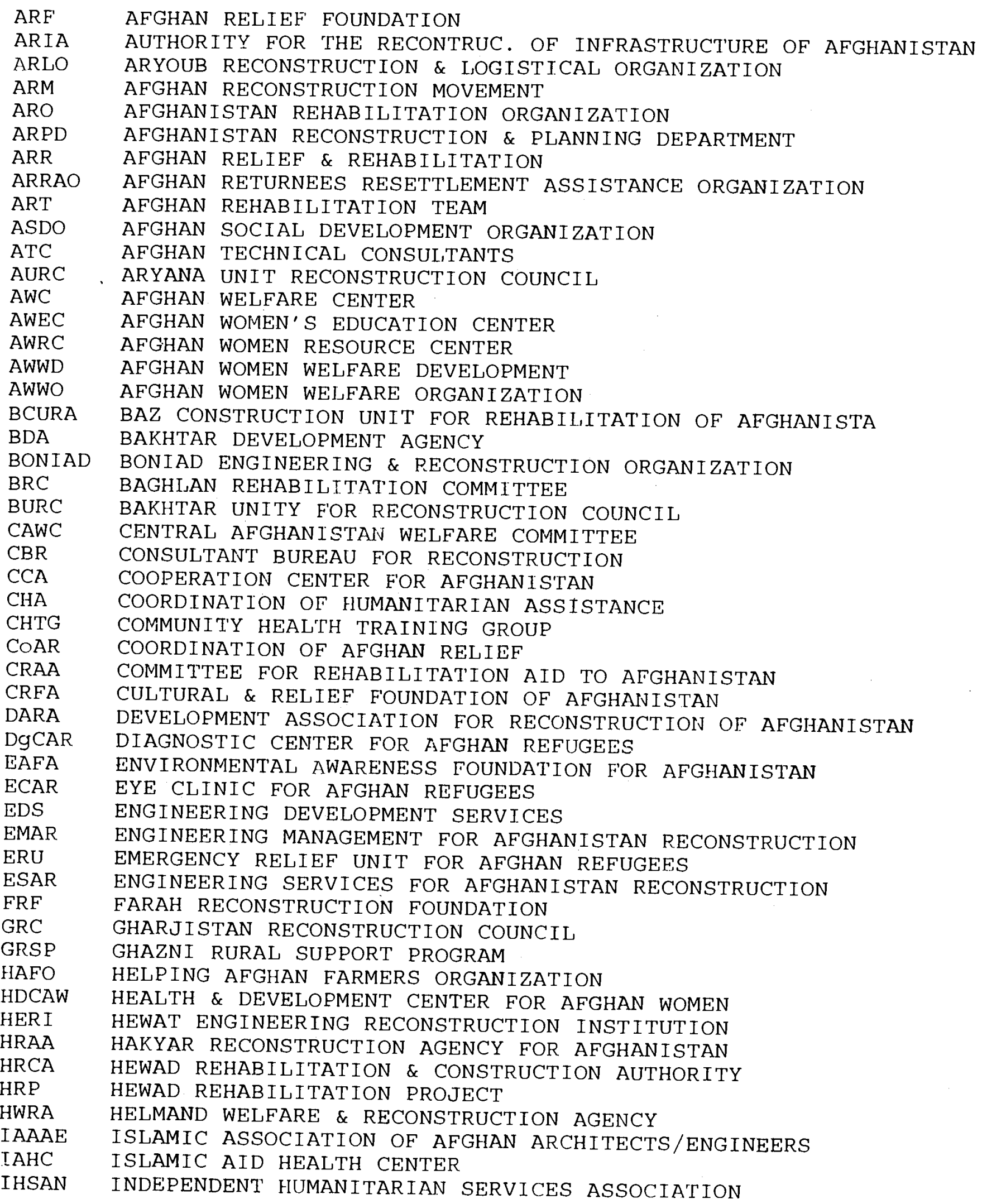




\section{AFGHANISTAN}

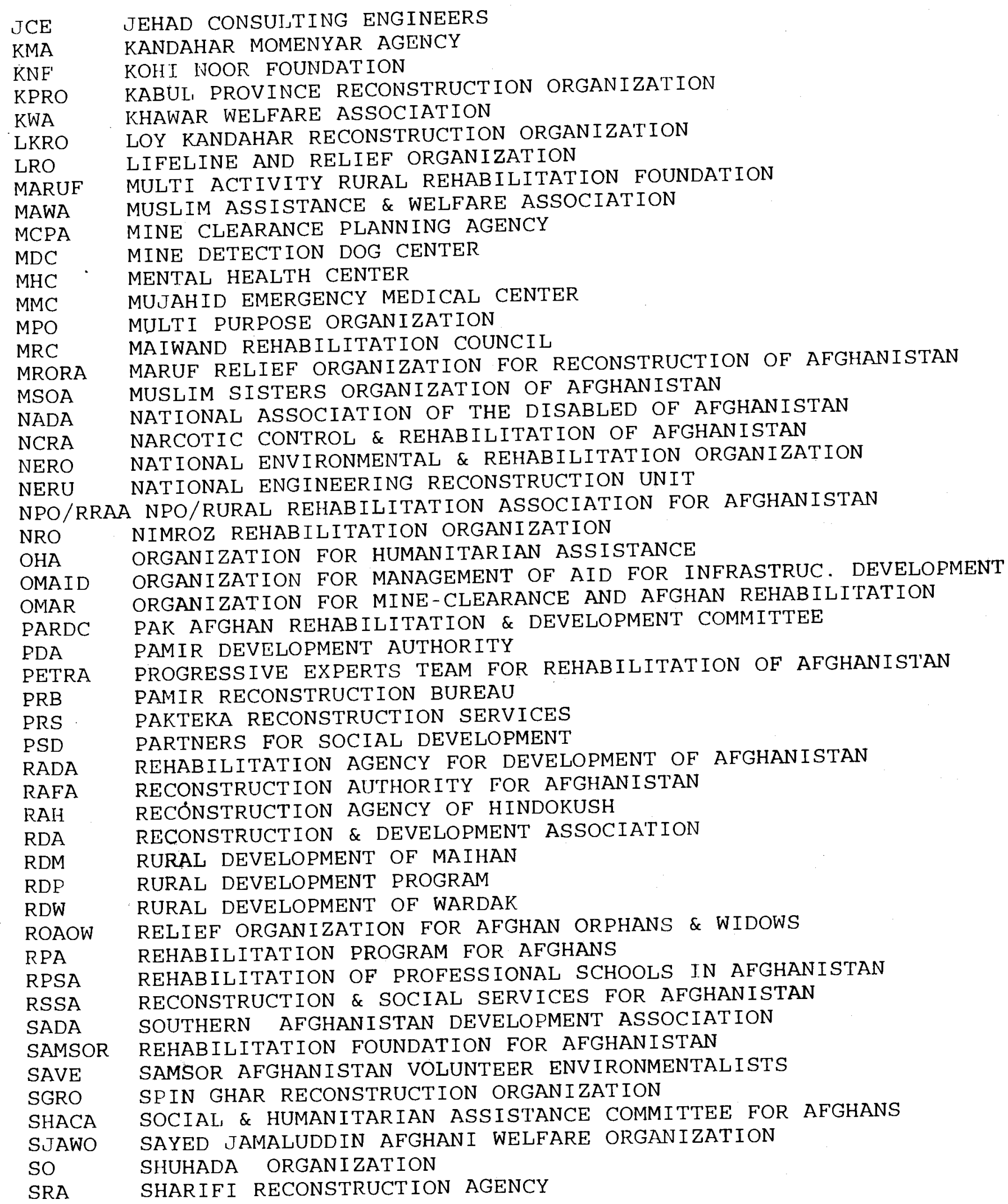




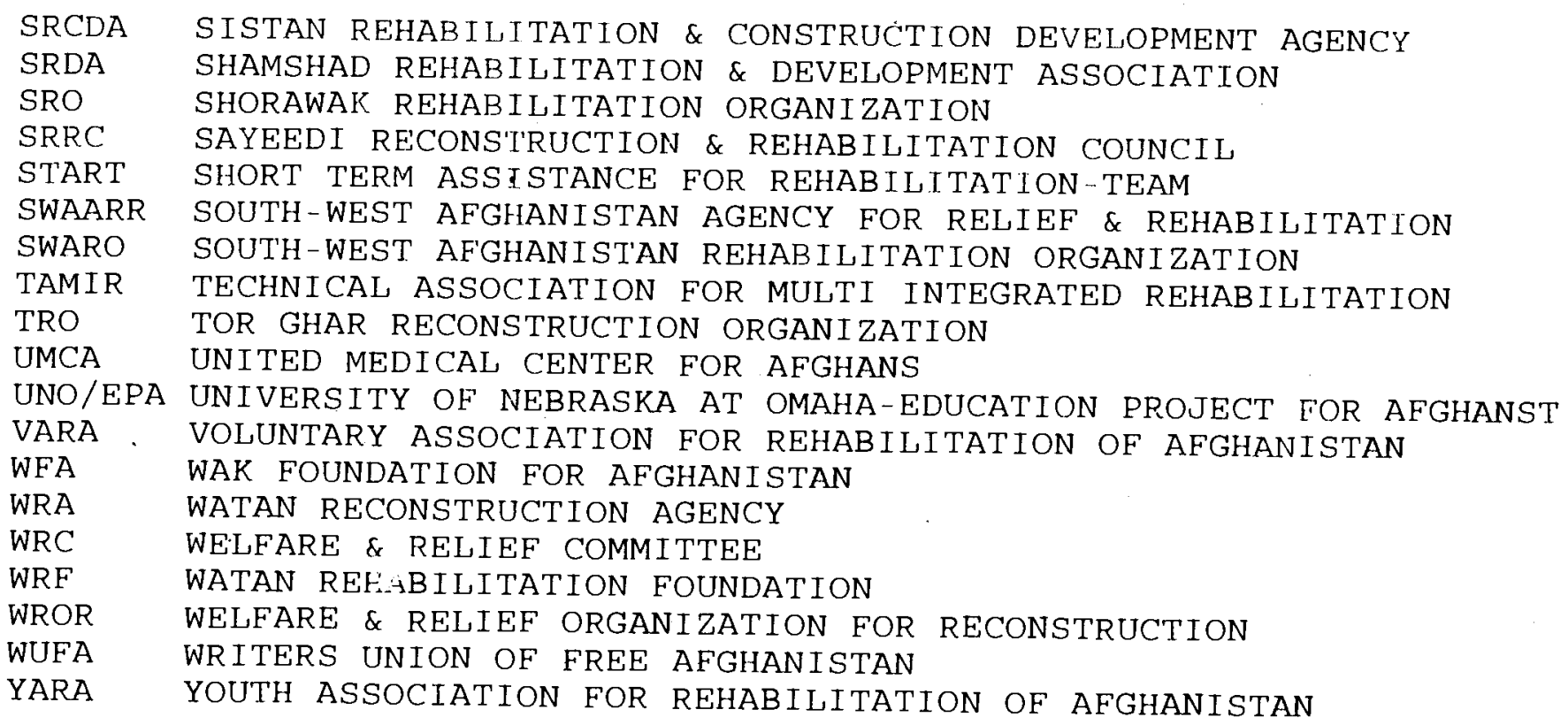

\section{AUSTRALLA}

1. SNI SHELTER NOW INTERNATIONAL

\section{AUSTRLA}

l. ARC

AUSTRIAN RELIEF COMMITTEE

\section{BELGIUM}

1. HI

HANDICAP INTERNATIONAL

2. SOS-PG SOS PG BELGIUM

\section{$\underline{C A N A D A}$}
I. $\mathrm{HCI}$
HUMAN CONCERN INTERNATIONAL

\section{DENMARK}
1. DACAAR
DANISH COMMITEE FOR AID IO AFGHAN REFUGEES

\section{FRANCE}

1. AICF ACTION INTERNATIONALE CONIRE LA FAIM

2. AFRANE AFRANE

3. AMIA AIDE MEDICALE INTERNATIONALE-AFGHANISTAN

4. AVICEN AFGHANISTAN VACCINATION/IMMUNIZATION CENTER

5. MADERA MISSION D' AIDE AU DEVELOPPEMENT DES ECONOMIES RURALES EN AFGHANISTAN

6. MDM MEDECINS DU MOND

7. MRCA MEDICAL REFRESHER COURSES FOR $\triangle F G H A N S$

8. PSF PHARMACIENS SANS FRONTIERES

9. SOLID SOLIDARITES 
1. AG-TTP AFGHAN-GERMAN TECHNICAL TRAINING PROGRAM

2. ANH AFGHANISTAN NOTHILFE

3. BEFARe BASIC EDUCATION FOR AFGHAN REFUGEES

4. GAA GERMAN AGRO ACTION

5. GAF GERMAN AFGHANISTAN FOUNDATION

6. HELP HELP-HILFE ZUR SELBS THILFE

7. ORA ORPHANS REFUGEES AND AID

8. UAAR UNION AID FOR AFGHAN REFUGEES

\section{ITALY}

1. ICD ITALIAN COOPERATION FOR DEVELOPMENT

JAPAN

1. JAMS JAPAN AFGHAN MEDICAL SERVICES

2. JIFF JAPANESE INTERNATIONAL WELFARE FOUNDATION

\section{$\underline{K U W A I T}$}

1. KRCS KUWAIT RED CRESCENT SOCIETY

2. LDI LAJNAT AL-DAWA AL-ISLAMIA

\section{NETHERLANDS}

1. DCA DUTCH COMMITTEE FOR AFGHANISTAN

2. HAF HELP THE AFGHANS FOUNDATION

\section{$\underline{N O R W A Y}$}

1. NAC NORWEGIAN AFGHIANISTAN COMMITTEE

\section{PAKISTAN}

\begin{tabular}{lll}
\hline 1. & CARP & CARITAS-AFGHANISTAN REFUGEE PROGRAM \\
2. & DS & DARUS SALAM \\
3. & IAU & IDARA AHYAUL ULUM \\
4. & MHC & MENTAL HEALTH CENTER \\
5. & Pak-CDP & Pak-COMMUNITY DEVELOPMENT PROGRAM \\
6. & PRCS & PAKISTAN RED CRESCENT SOCIETY
\end{tabular}

$Q A T A R$

I. LQO LAJNAT AL-QATAR FOR ORPHANS

\section{$\underline{S A U D I A R A B L A}$}

1. ARCON AFGHANISTAN RECONSTRUCTION CONSULTANTS

2. IIRO ISLAMIC INTERNATIONAL RELIEF ORGANIZATION

3. LBI LAJNAT AL-BIRR AL-ISLAMIA

4. MF MOWAFAQ FOUNDATION

5. SRCS SAUDI RED CRESCENT SOCIETY

$\underline{S U D A N}$

1. ISRA ISLAMIC RELIEF AGENCY 
1. RBS RADDA BARNEN-SWFDISH SAVE TIIE CIILDREN

2. SCA SWEDISH COMMITIEE FOR AFGHIANISTAN

\section{SWITZERLAND}

1. ATA/AP ANT1-TB ASSOCIATION/AFGHANISTAN PROGRAM

2. ICRC INTERNATIONAL COMMITTEE OF THE RED CROSS

3. SAA SWISS AID FOR AFGHANISTAN

4. TDH TERRE DES HOMMES

\section{UNITED ARAB EMIRATES}

1. UAE-RCS UNITED ARAB EMIRATES-RED CRESCENT SOCIETY

UNITED KINGDOM

1. A-AID AFGHANAID

2. BAT BRITISH AFGHAN TRUST

3. GP-UK GLOBAL PARTNERS-UK

4. HT HALO TRUST

5. JWMM JACOB'S WELL MEDICAL MISSION

6. MAI MUSLIM AID-UK

7. MERLIN MEDICAL EMERGENCY RELIEF INTERNATIONAL

8. OV THE OCKENDEN VENTURE

9. OXFAM OXFAM

10. SCF-UK SAVE THE CHILDREN FUND-UK

11. SGAA SANDY GALL AFGIIANISTAN APPEAL

12. SERVE SERVING EMERGENCY RELIEF \& VOCATIONAL ENTERPRISES

13. VSO VOLUNTARY SERVICES ORGANIZATION

\section{UNITED STATES}

1. AABRAR AFGHAN AMPUTEE BICYCLISTS FOR REHAB. \& RECREATION

2. IMC INTERNATIONAL MEDICAL CORPS

3. IOC INTERNATIONAL ORPHANS CARE

4. IRC INTERNATIONAL RESCUE COMMITTEE

5. $\mathrm{MCl}$ MERCY CORPS INTERNATIONAL

6. SC-US SAVE THE CHILDREN FEDERATION-US

\section{INTERNATIONAL}

I. CARE CARE INTERNATIONAL

2. IAM INTERNATIONAL ASSISTANCE MISSION

3. IFRCS INT"L FEDERATION OF RED CROSS/ CRESCENT SOCIETIES

4. IOM INTERNATIONAL ORGANIZATION

5. MSF MEDECINS SANS FRONTIERES-INTERNATIONAL 


\section{LOCATION CODES}

0100 KABUL PROVINCE

$\begin{array}{ll}0101 & \text { Kabul } \\ 0102 & \text { Bagrami } \\ 0103 & \text { Khake Jabar } \\ 0104 & \text { Chardehi } \\ 0105 & \text { Charasyab (Chrasyab) } \\ 0107 & \text { Sarobi } \\ 0108 & \text { Paghman } \\ 0109 & \text { Shakar Dara } \\ 0110 & \text { Mir Bachakot (Kohdaman) } \\ 0111 & \text { Kalakan } \\ 0112 & \text { Qara Bagh } \\ \text { 1113 } & \text { Istalef. }\end{array}$

\section{KAPISA PROVINCE}

$\begin{array}{ll}0201 & \text { Kapisa (Mahmoud Raqi) } \\ 0202 & \text { Tagab } \\ 0203 & \text { Alasay (Alasai) } \\ 0204 & \text { Kohistan } \\ 0205 & \text { Kohband (Durnama) } \\ 0206 & \text { Nejrab } \\ 0207 & \text { Panjshare (Rokha) } \\ 0208 & \text { Panjshare1 (Zenya/4 Qarya) } \\ 0209 & \text { Panjshare2(Dara Hazara) }\end{array}$

\subsection{PARWAN PROVINCE}

$\begin{array}{ll}0301 & \text { Charikar } \\ 0302 & \text { Ghorband (Syahgerd) } \\ 0303 & \text { Shenwari (Shinwar) } \\ 0304 & \text { Bagram } \\ 0305 & \text { Kohe Safi } \\ 0306 & \text { Jabul Saraj (Jabulsaraj) } \\ 0307 & \text { Salang } \\ 0308 & \text { Surkh Parsa (Surkhi Parsa) } \\ 0309 & \text { Shekh Ali (Kotae Ashro) }\end{array}$

\section{MAIDAN/WARDAK PROVINCE}

$\begin{array}{ll}0401 & \text { Maydan Shar } \\ 0402 & \text { Jalrez } \\ 0403 & \text { Nerkh } \\ 0404 & \text { Chak } \\ 0405 & \text { Day Mirdad } \\ 0406 & \text { Markaz Besud (Behsud) } \\ 0407 & \text { Gardandewal (Behsud Part1) } \\ 0408 & \text { Syed Abad } \\ 0409 & \text { Jaghatu - boundaries not } \\ & \quad \text { on map }\end{array}$

\section{LOGAR PROVINCE}

$\begin{array}{ll}0501 & \text { Baraki } \\ 0502 & \text { Khoshi } \\ 0503 & \text { Charkh } \\ 0504 & \text { Mohd Agha (Mohammad Agha) } \\ 0505 & \text { Azra (Azro) } \\ 0506 & \text { Puli Alam (Kulangar) }\end{array}$

\section{GHAZNI PROVINCE}

$\begin{array}{ll}0601 & \text { Ghazni (Khazni) } \\ 0602 & \text { Zena Khan } \\ 0603 & \text { Deh Yak (Ramak) } \\ 0604 & \text { Khwaja Omri (Khwaja Omari) } \\ 0605 & \text { Nawor } \\ 0606 & \text { Jaghatu/Waiz } \\ 0607 & \text { Jaghori } \\ 0608 & \text { Malestan } \\ 0609 & \text { Qarabagh } \\ 0610 & \text { Moqor } \\ 0611 & \text { Gelan } \\ 0612 & \text { Ab Band } \\ 0613 & \text { Andar (Miri. Shelgar) } \\ 0614 & \text { Giro } \\ 0615 & \text { Nawa }\end{array}$

\section{PAKTEKA PROVINCE}

$\begin{array}{ll}0616 & \text { Katawaz (Zarghun Shahr) } \\ 0617 & \text { Sultan Khan (Yahya Khel) no } \\ & \text { on the map } \\ 0618 & \text { Jani Khel not on the map } \\ 0619 & \text { Dila (Khoshamand) } \\ 0620 & \text { Omna } \\ 0621 & \text { Sharan } \\ 0622 & \text { Yusuf Khel -not on the map } \\ 0623 & \text { Wazakhwa } \\ 0624 & \text { Wol-Mamay } \\ 0702 & \text { Urgoun } \\ 0703 & \text { Neka } \\ 0704 & \text { Sarhawza (Sultani) } \\ 0705 & \text { Gayan } \\ 0706 & \text { Barmal } \\ 0708 & \text { Zelook } \\ 0722 & \text { Gomal } \\ 0723 & \text { Sarobi } \\ 0732 & \text { Mata Khel (Mata Khan) }\end{array}$




$\begin{array}{ll}0701 & \text { Gardaiz } \\ 0707 & \text { Sperah (Matoon) } \\ 0709 & \text { Khost (Mwul) } \\ 0710 & \text { Mandozi (Dwi (Saroti) } \\ 0711 & \text { Sabari } \\ 0712 & \text { Trayzai (Alisher) } \\ 0713 & \text { Dara Darang } \\ 0714 & \text { Tani (Tunni) } \\ 0715 & \text { Gurbuz (Gruiz) } \\ 0716 & \text { Nader Shah (Keray/Almara) } \\ 0717 & \text { Mosa Khel (Zor Kot) } \\ 0718 & \text { Qalandar } \\ 0719 & \text { Jaji (Ali Khel) } \\ 0720 & \text { Hasan Khel (Ahmad Khel) } \\ 0721 & \text { Jani Khel } \\ 0724 & \text { Jadran (Waze) } \\ 0725 & \text { Shawak (Bargay) } \\ 0726 & \text { Shamal Karam } \\ 0727 & \text { Sayed Karam } \\ 0728 & \text { Chamkani } \\ 0729 & \text { Dand Patan } \\ 0730 & \text { Lajmangal (Laza) } \\ 0731 & \text { Zormat } \\ 0733 & \text { Wolma (Orma) } \\ 0734 & \text { Jaji Maidan } \\ 0735 & \text { Bak (Barbay) }\end{array}$

\section{NANGARHAR}

$\begin{array}{ll}0801 & \text { Jalalabad } \\ 0802 & \text { Shinwar (Ghani Khel) } \\ 0803 & \text { Dorbaba } \\ 0804 & \text { Bati Kot } \\ 0805 & \text { Rodat (Shahi Kot) } \\ 0806 & \text { Chaparhar (Chapleyar) } \\ 0807 & \text { Mohmand Dara (Basa Wul) } \\ 0808 & \text { Lalpur } \\ 0809 & \text { Naziyan (Naziuan) } \\ 0810 & \text { Deh Bala } \\ 0811 & \text { Achin } \\ 0812 & \text { Kama } \\ 0813 & \text { Goshta (Kheaja Zewar) } \\ 0814 & \text { Sorkh Rod } \\ 0815 & \text { Behsud } \\ 0816 & \text { Hesarak } \\ 0817 & \text { Kouz Konar (Shewa) } \\ 0818 & \text { Darae Noor } \\ 0819 & \text { Khogiani (Kaja) } \\ 0820 & \text { Pachier/Agam (Wagam) } \\ 0821 & \text { Sherzad (Mama Khel) }\end{array}$

$\begin{array}{ll}0901 & \text { Mehterlam } \\ 0902 & \text { Qarghaie (Katz Aziz Khan) } \\ 0903 & \text { Dawlatsha (Dawlat Shah) } \\ 0904 & \text { Alingar (Alinegar) } \\ 0905 & \text { Alishing (Ali Shang) } \\ 0906 & \text { Nooristan }\end{array}$

\section{KUNAR PROVINCE}

$\begin{array}{ll}1001 & \text { Chaghasarai (Asadabad) } \\ 1002 & \text { Chapa Dara } \\ 1003 & \text { Naranj/Badil (Narang) } \\ 1004 & \text { Naray } \\ 1005 & \text { Dongom (Dangam) } \\ 1006 & \text { Khas Kunar } \\ 1007 & \text { Bar Kunar (Asmar) } \\ 1008 & \text { Sirkanay } \\ 1009 & \text { Kamdesh } \\ 1010 & \text { Bargey Metal (Bargi Matal) } \\ 1011 & \text { Chawki } \\ 1012 & \text { Nour Gul (Mazar) } \\ 1013 & \text { Pech (Dara Pech) }\end{array}$

\section{BADAKHSHAN PROVINCE}

$\begin{array}{ll}1101 & \text { Faizabad } \\ 1102 & \text { Ragh } \\ 1103 & \text { Shar Bozurg } \\ 1104 & \text { Darwaz (Nesay) } \\ 1105 & \text { Khwahan } \\ 1106 & \text { Jurm } \\ 1107 & \text { Baharak } \\ 1108 & \text { Keranomonjan } \\ 1109 & \text { Eshkashem } \\ 1110 & \text { Zebak } \\ 1111 & \text { Sheghnan } \\ 1112 & \text { Keshem } \\ 1113 & \text { Wakhan (Khandud) }\end{array}$

\section{TAKHAR PROVINCE}

$\begin{array}{ll}1201 & \text { Taloqan } \\ 1202 & \text { Rustaq } \\ 1203 & \text { Khwajaghar } \\ 1204 & \text { Eshkamesh } \\ 1205 & \text { Bangi } \\ 1206 & \text { Chal } \\ 1207 & \text { Chah Ab } \\ 1208 & \text { Yangi Qala } \\ 1209 & \text { Darqad } \\ 1210 & \text { Farkhar } \\ 1211 & \text { Warsaj } \\ 1212 & \text { Kalafgan }\end{array}$




\section{BAGHLAN PROVINCE}

1301 Baghlan (Baghlan Jadeed)

1302 Puli Khumri

1303 Dahanae Ghri

1304 Doshi

1305 Tala Brfk

1306 Khenjan

1307 Nahrein

1308 Burka

1309 Anderab (Banu)

1310 Khost Fring

\section{KUNDUZ PROVINCE}

$\begin{array}{ll}1401 & \text { Kunduz } \\ 1402 & \text { Aliabad } \\ 1403 & \text { Hazrat Imam (Imam Sahib) } \\ 1404 & \text { Khanabad } \\ 1405 & \text { Archi (Dasht Archi) } \\ 1406 & \text { Qala Zal } \\ 1407 & \text { Char Dara }\end{array}$

\section{SAMANGAN PROVINCE}

$\begin{array}{ll}1501 & \text { Samangan (Aibak) } \\ 1502 & \text { Dara Souf } \\ 1503 & \text { Khulm (Tashqurghan) } \\ 1504 & \text { Kaldar } \\ 1505 & \text { Hazrat Sultan } \\ 1506 & \text { Roy Doab }\end{array}$

\section{BALKH PROVINCE}

$\begin{array}{ll}1601 & \text { Mazar Sharif } \\ 1602 & \text { Balkh } \\ 1603 & \text { Nahr Shahi } \\ 1604 & \text { Dehdadi } \\ 1605 & \text { Char Kant } \\ 1606 & \text { Dowlatabad } \\ 1607 & \text { Sholgera (Boyna Qara) } \\ 1608 & \text { Keshende (Aq Kapruk) } \\ 1609 & \text { Shortepa } \\ 1610 & \text { Char Bolak } \\ 1611 & \text { Chemtal }\end{array}$

\section{JAWZJAN PROVINCE}

$\begin{array}{ll}1701 & \text { Sheberghan } \\ 1702 & \text { Sang Charak } \\ 1703 & \text { Balkhab } \\ 1704 & \text { Sari Pul } \\ 1705 & \text { Kohistanat (Mahtanat) } \\ 1706 & \text { Aqcha } \\ 1707 & \text { Mardyan } \\ 1708 & \text { Faizabad } \\ 1709 & \text { Khanaqah } \\ 1710 & \text { Qarqin } \\ 1711 & \text { Khamyab } \\ 1712 & \text { Mengajek }\end{array}$

\section{FARYAB PROIINCE}

$\begin{array}{ll}1801 & \text { Maymana (Mimana) } \\ 1802 & \text { Andkhoy } \\ 1803 & \text { Khan Charb. } \\ 1804 & \text { Qarghan - not on map } \\ 1805 & \text { Qaramqul } \\ 1806 & \text { Pashtoon Kot } \\ 1807 & \text { Kohistan (Lawlash) } \\ 1808 & \text { Almar } \\ 1809 & \text { Belcheragh } \\ 1810 & \text { Shirin Tagab } \\ 1811 & \text { Qaysawr } \\ 1812 & \text { Darzab } \\ 1813 & \text { Dowlatabad }\end{array}$

\section{BADGHIS PROVINCE}

$\begin{array}{ll}1901 & \text { Qala Nau } \\ 1902 & \text { Kushk Kohna } \\ 1903 & \text { Morghab } \\ 1904 & \text { Ghormach } \\ 1905 & \text { Qades } \\ 1906 & \text { Jawand }\end{array}$

\section{HERAT PROVINCE}

$\begin{array}{ll}2001 & \text { Herat } \\ 2002 & \text { Enjeel (Anjil) } \\ 2003 & \text { Gozarah } \\ 2004 & \text { Pashtun Zar. } \\ 2005 & \text { Gulran } \\ 2006 & \text { Kushk } \\ 2007 & \text { Ghoryan } \\ 2008 & \text { Obey (Obe) } \\ 2009 & \text { Chesht Sharif (Chest Sha.) } \\ 2010 & \text { Karokh } \\ 2011 & \text { Zendajan (Fau Shang) } \\ 2012 & \text { Kohsan } \\ 2013 & \text { Adreskan (Adraskan) }\end{array}$

\section{FARAH PROVINCE}

$\begin{array}{ll}2101 & \text { Farah } \\ 2102 & \text { Khak Safid } \\ 2103 & \text { Shindand (Sabzwar) } \\ 2104 & \text { Farsi } \\ 2105 & \text { Anar Dara } \\ 2106 & \text { Bakwa (Sultan Bakwa) } \\ 2107 & \text { Bala Balouk. } \\ 2108 & \text { Qala Kah } \\ 2109 & \text { Gulistan } \\ 2110 & \text { Purchaman } \\ 2111 & \text { Lash Jaween - formerly in } \\ & \text { Nimroz province, and appear: } \\ & \text { on Nimroz map }\end{array}$




$\begin{array}{ll}2201 & \text { Zaranj } \\ 2202 & \text { Kang/Kurki (Miel Kurki) } \\ 2203 & \text { Asle Chakhansur } \\ 2204 & \text { Khosh Rod (Lokhi, Khas) } \\ 2205 & \text { Char Borjak }\end{array}$

\section{HELMAND PROVINCE}

$\begin{array}{ll}2301 & \text { Bust (Lashkargah) } \\ 2302 & \text { Reg (Khanneshin) } \\ 2303 & \text { Nahr Saraj (Greshk) } \\ 2304 & \text { Sarban Qala (Sangin) } \\ 2305 & \text { Mosa Qala } \\ 2306 & \text { Kajaki } \\ 2307 & \text { Nauzad } \\ 2308 & \text { Washare } \\ 2309 & \text { Garmser (Darweshan) } \\ 2310 & \text { Nad-Ali } \\ 2311 & \text { Nawae Barakzai (Hazaras) } \\ 2312 & \text { Baghran } \\ 2313 & \text { Deshu }\end{array}$

\section{KANDAHAR PROVINCE}

$\begin{array}{ll}2401 & \text { Kandahax } \\ 2402 & \text { Spin Boldak } \\ 2403 & \text { Shega } \\ 2404 & \text { Dand } \\ 2405 & \text { Daman } \\ 2406 & \text { Axghistan } \\ 2407 & \text { Khakxaiz } \\ 2408 & \text { Ghorak } \\ 2409 & \text { Nesh } \\ 2410 & \text { Shorabak (Goaran) } \\ 2411 & \text { Reg (Karez) } \\ 2412 & \text { Arghandab } \\ 2413 & \text { Panjwai } \\ 2414 & \text { Maywand (Keshke Nakhud) } \\ 2415 & \text { Shah Wali Kot (Dahla) } \\ 2416 & \text { Maruf }\end{array}$

$\begin{array}{ll}2501 & \text { Qalat } \\ 2502 & \text { Jaldak (Shahr Safa) } \\ 2503 & \text { Mizan } \\ 2504 & \text { Shah Joy } \\ 2505 & \text { Arghandab } \\ 2506 & \text { Dai Chopan } \\ 2507 & \text { Shinkai } \\ 2508 & \text { Atghar } \\ 2509 & \text { Shemalzai }\end{array}$

\section{ORUZGAN PROVINCE}

$\begin{array}{ll}2601 & \text { Tirin (Tirin Kot) } \\ 2602 & \text { Oruzgan (Khosh) } \\ 2603 & \text { Deh Raud } \\ 2604 & \text { Dai Kundi (Khadir) } \\ 2605 & \text { Shahristan } \\ 2606 & \text { Chorah } \\ 2607 & \text { Ajrestan } \\ 2608 & \text { Gezab } \\ 2609 & \text { Kajran }\end{array}$

\section{GHOR PROVNCE}

$\begin{array}{ll}2701 & \text { Chaghcharan } \\ 2702 & \text { Shahrak } \\ 2703 & \text { Saghar } \\ 2704 & \text { Toulak (Toolak) } \\ 2705 & \text { Taywara } \\ 2706 & \text { Lal Surjangal } \\ 2707 & \text { Pasaband }\end{array}$

\section{BAMYAN PROVNCE}

$\begin{array}{ll}2801 & \text { Bamyan } \\ 2802 & \text { Shibar (Shumbul) } \\ 2803 & \text { Kahmard } \\ 2804 & \text { Saighan } \\ 2805 & \text { Yakowlang } \\ 2806 & \text { Panjab } \\ 2807 & \text { Waras }\end{array}$




\begin{tabular}{|c|c|c|c|}
\hline ID & 5 B, Gulmohar Rd, U/T, Peshawar & Wynn & 30 \\
\hline D & Abu Noman Plaza, J. Rd, GPO.Box 299, Pesh. & F. Marranay & \\
\hline $\begin{array}{l}\text { RAR } \\
\text { RP }\end{array}$ & 106, Gul Haji Plaza, J. RD, Peshawar & Abdul Baseer & 42417 \\
\hline $\begin{array}{l}\text { CRP } \\
\text { EA }\end{array}$ & 856, Street 30, D/4, Hayatabad, Peshawar & Eng: Dadullah & 21 \\
\hline $\begin{array}{l}\text { AAEA } \\
\text { AAID }\end{array}$ & $\begin{array}{l}\text { 30, 3rd Floor, Spinzar Plaza, J.Rd Pesh. } \\
26 \text {, Canal Lane, U/T, Peshawar }\end{array}$ & Naqib-u-Rahman & \\
\hline $\begin{array}{l}\text { AAID } \\
A C A\end{array}$ & $\begin{array}{l}\text { Ganal Lane, U/T, Peshawar } \\
\text { Gul Haji Plaza, J. Rd, Peshawar }\end{array}$ & A. Hai Warshan & 4 \\
\hline & $\begin{array}{l}\text { 128, Gul Haji Plaza, J. Rd, Peshawar } \\
\text { Opp. T.T.I, Sarobi Rd Jalalabad Afghanis. }\end{array}$ & $\begin{array}{l}\text { Bashir Ahmad } \\
\text { Eng. Najeeb }\end{array}$ & $\begin{array}{l}8413 \\
\mathrm{Nil}\end{array}$ \\
\hline A & N/A-A, Khalil Town, GPO Box 858, Peshawar. & & \\
\hline CLU & $\begin{array}{l}\text { 6-A, Khalil Town, GPO Box } 858 \text {, Peshawar. } \\
123 / 123-A \text {, Industrial Area, Hayatabad Pesh. }\end{array}$ & Gul Wai & \\
\hline & $\begin{array}{l}\text { 123/123-A, Industrial Area, Hayatabad Pesh. } \\
25-\mathrm{B} \text {. Rahman Baba Rd, UPO Box } 849 \text {, Peshawar }\end{array}$ & $\begin{array}{l}\text { ENG. M. Ka } \\
\text { Ruhani War }\end{array}$ & $\begin{array}{l}8 \\
4\end{array}$ \\
\hline $\mathrm{O}$ & $\begin{array}{l}\text { 2b-B. Ranman Bc } \\
\text { N/A } \\
432\end{array}$ & Ini War & \\
\hline & $\begin{array}{l}\text { 432, Old Bara Rd, U/T, UPO Box } 922, \mathrm{E} \\
\text { Plot 5, Murad Plaza, Universt. Rd, Pe }\end{array}$ & opal & \\
\hline & $\begin{array}{l}\text { Plot 5, Murad Plaza, Universt. Rd, Pesh. } \\
\text { Golmemdy Chowk, Catenra Rd, PO Box } 468 \text {, Quetta }\end{array}$ & aruddin & \\
\hline $\mathrm{O}$ & Chowk, Catenra Rd, PO Box 4 & Baridad Atif & 83 \\
\hline $\mathrm{P}$ & $\begin{array}{l}\mathrm{N} / \mathrm{A} \\
\text { Flat } 23\end{array}$ & $\begin{array}{l}\text { Us } \\
\mathrm{Ab}\end{array}$ & $\begin{array}{l}28 \\
86\end{array}$ \\
\hline$S$ & UPO Box 1463 , University Town, Peshawar & $\begin{array}{l}\text { Abdull. Sada } \\
\text { A. R. Sahak }\end{array}$ & 286 \\
\hline A & Hada-i-Torkham Torkham Rd, Jalalabad Afgh. & $\begin{array}{l}\text { A. R. Sandk } \\
\text { Eng. M. Tahir }\end{array}$ & \\
\hline IF & 84-E Rahman Baba Rd, U/T, Peshawar & S. Zahine & \\
\hline 20 & $\begin{array}{l}\text { N/A } \\
\text { Mehtarlam Laghman Afghani }\end{array}$ & & \\
\hline$S$ & $\begin{array}{l}\text { Mehtarlam Laghman Afghanistan(Tel.c/o ARDI } \\
133 \text { Gul Haji Plaza, GPO Box 615, Peshawar }\end{array}$ & Shah & 84 \\
\hline TTP & $\begin{array}{l}133 \text { Gul Haji Plaza, GPO Box 615, Peshawar } \\
23 \text {, K.K.K Rd, U/T Peshawar }\end{array}$ & Aziz Or & \\
\hline $\mathrm{S}$ & $\begin{array}{l}23, \mathrm{~K} . \mathrm{K} . \mathrm{K} \mathrm{Rd}, \mathrm{U} / \mathrm{T} \text { Peshaw } \\
178, \mathrm{D} / 1, \mathrm{Ph} .1, \text { Hayatabad, }\end{array}$ & id $N$ & \\
\hline 12 & $\begin{array}{l}178, \mathrm{D} / 1 \text {, Ph. 1, Hayatabad, GPO Box } 631 \text { Pesh. } \\
1426-\mathrm{T} \text { Old Bara Rd, UPO Box } 753 \text {, Peshawar }\end{array}$ & $z Q \bar{c}$ & 51 \\
\hline ? ? & $\begin{array}{l}\text { 1426-T Old Bara Rd, UPO Box 753, Peshawar } \\
18-\mathrm{E} \text {, S.J. Afghani Rd, U/T, Peshawar }\end{array}$ & Jasim & \\
\hline EC & $\begin{array}{l}\text { 18-E, S.J. Afghani Rd, U/T, Peshawar } \\
\text { 7-B, 3rd Floor, Zarak Plaza, J. Rd Pesha }\end{array}$ & $\mathrm{m} \mathrm{Me}$ & \\
\hline$F$ & $\begin{array}{l}\text { 7-B, 3rd Floor, Zarak Plaza, J. Rd Peshawal } \\
\text { Wazir Akbar Khan Kabul Afghanistan }\end{array}$ & onnet & \\
\hline A & $\begin{array}{l}\text { Wazir Akbar Khan Kabul Afghanistan } \\
109 \text {, Canal Rd, U/T, UPO Box 644, Peshawar }\end{array}$ & onnet & $\begin{array}{l}20 \\
42\end{array}$ \\
\hline$M$ & $15 \mathrm{~F} / \mathrm{B}$ K.K.K Rd, U/T, GPO Box 1117 Peshawar & Mohd St & $\begin{array}{l}42 \\
44\end{array}$ \\
\hline MA & 21, Old Jamrod Rd, U/T, Peshawar & Dr. Zar & 12 \\
\hline$[-7$ & 10, Park Avenue U/T, GPO Box 326 Peshawar & Remy $\mathrm{R}$ & 33 \\
\hline RAN & 306, 3rd Floor, Gul Haji Plaza,J.Rd, Pesh. & am & $\begin{array}{l}31 \\
79\end{array}$ \\
\hline C & 2, Canal Bank Rd, U/T Peshawar & Haji D & $\begin{array}{l}44279 \\
41691\end{array}$ \\
\hline AAR & Town Peshawar & & \\
\hline AR & Flat 315,3 rd Floor, Gul Haji Plaza, & $S \in$ & \\
\hline DGH & bad, Canal Rd, GPO Box 679 , U/T F & Wahidi & \\
\hline DGH & J Afghani Rd, U/T, GPO BOX 448, Pesh & $\mathrm{Dr} \cdot \mathrm{Q} \cdot \mathrm{Mu}$ & $\begin{array}{l}8 \\
4\end{array}$ \\
\hline (2) & $\mathrm{Rd}, \mathrm{U} / \mathrm{T}, \mathrm{P} . \mathrm{O} . \mathrm{Box}$ 865, Peshawar & Eng. A. & $\begin{array}{l}4 \\
4\end{array}$ \\
\hline & ishabad Rd, Peshawar & Hakim Ta & $\begin{array}{l}15 \\
49\end{array}$ \\
\hline NO & cha House, New Arl & Eng.R. & $\begin{array}{l}49 \\
31\end{array}$ \\
\hline & 918-T, Arbab Col. Belal Lane, Arbab Rd, Pesh. & Pro.G. & $\begin{array}{l}41031 \\
44263\end{array}$ \\
\hline & $\mathrm{N} / \mathrm{A}$ & & \\
\hline $\mathrm{ADA}$ & Pul, Dehry Rd, U/T. GPO 644 Pesh. & Mc & \\
\hline & ar Lane, U/T, GPO Box 489, Peshawar & Eng. E & $\begin{array}{l}45 \\
84\end{array}$ \\
\hline $\mathrm{CAF}$ & nzar Plaza, Jahangirabad Peshawar & Dr.M. Humayun & 44986 \\
\hline 70 & 171, Sector 2, Gulaee Araban, Jalalabad, Afgh. & Homayu & $\mathrm{Nil}$ \\
\hline$r 01$ & 25-B, Park Avenue, PO Box 991, U/T Peshawar & A.F.Mu & 840241 \\
\hline & Shafa Khana Qwai Markaz, Kabul Afgha & Dr. $\mathrm{Ha}$ & 44916 \\
\hline & 184, Uppẽr Canal Lane, U/T, Peshawar & M. K. Rasheed & 842189 \\
\hline
\end{tabular}


ARDCD

Mohallai Jambilan Tahkal Bala, Peshawar

M. Khalil

42929

ARDO

ARDP

Behind New Daramsal, Regshamt Khan Jal.Afgh.

. Ghaus Haida

4325

AREA - 1

414, 4th Fl, Gul Haji Plaza, UPO 937, Pesh.

Dr. Sediq Asif

44750

AREA- 2 17-E Abdara Rd, U/T, Peshawar

Eng. R. Ludin

41993

ARF

Chowk-i-Mukhaberat, Jalalabad Afghanistan

ARIA

42 , St. 5, G-3, Ph. 2, Hayatabad, Peshawar

ARLO

57, Gulshan Iqbal Town Univers.Rd, Pesh.

Mohd. Yousuf

$\mathrm{Nil}$

ARM

ARO

ARPD

ARR

ARRAO

Murad Plaza, Grd Flr, Tamboan Stop; Peshawar $\mathrm{N} / \mathrm{A}$

179, D-1, Phase 1, Hayatabad, Peshawar

S. I. Gallani 811261

Dr.Faroq Azam 840539

Haji Sediqi 843096

A.Malik Ihsan 442922

45, 2nd Floor, Shama Shoping Center, Pesh. 402/403, Gul Haji Plaza, Univers.Rd, Peshawar

ART

ASDO

Char Rahi Marastun, Jalalabad, Afghanistan 283,B-2, Near Mini Market, S/T Quetta

$\mathrm{ATA} / \mathrm{AP}$ 305, 3rd Floor, Gul Haji Plaza,J. Rd, Pesh.

ATC

AURC 4, 1st Floor, Khyber View Paza, Peshawar 45/D-4, Old J. Rd, U/T, GPO Box 1149, Pesh. 171, Block 3, Street-J, S/Town, Quetta

AVICEN 22 K.K.K Rd, UPO Box 992, Peshawar

AWC

AWEC 3, st. 9, Nasir Bagh Rd, Peshawar

AWRC 195, St. 57, G-9/4, Islamabad

Azimul. Naizi

811579

Hassan Qadri

Abdul Aziz

$\mathrm{Ni} 1$

Abdul Wakeel

843255

Khalid Farahi

Shabiani

2013

Dr. Ayub zhian

K. Eblagh

444090

840378

Ni amatullah

440126

Dr. Siddiqi

Pashtunyar

41308

442922

45279

Ms. Mahbooba

842671

Mumtaz Ali Shan H.Osmania L.Arbab Rd, Pesh. Najia Zewari

AWWD A/1, Gura Qabrista Opp. of Police Chawk, Pesh. M

AWWO

BCURA 141, D-3, Ph. 1, Hayatabad, Peshawar

Ms. Jamila

859489

841552

843497

Ms. Zuhra

810722

300, St. 25, D-4, Ph. 1, Hayatabad, Peshawar

BDA

BEFARe 209, 2nd Floor Gul Haji Plaza, J.Rd, Pesh.

Eng. Imam

813935

M. H.Jalalzai 44296

BONIAD 8 Tatara Rd, Rahatabad, P.O.Box 1481, Pesh.

BRC

BURC

CARE

CARP

CAWC

CBR

CCA

CHA

CHTG

COAR

CRAA

CRFA

DACAAR

16 Old Bara Road, U/T, PO Box 1461, Pesh.

Michael Hirth

589 , St. 30,D-4, Ph. 1, Hayatabad, Peshawar

Kassi Rd, C/O B. Hospital, Quetta

A. Najeeb

840631

6, Park Lane, Park Rd, U/T, PO 926, Pesh. 17/F-1 K.K.K Rd, U/T, GPO Box 581, Peshawar 272, St 12, D-3, Ph 1, Hayatabad Peshawar 19 Canal Bank Rd, U/T, GPO Box 264, Pesh.

Mohd Haider

40889

S. A. Malik

814421

Paul Barker

75439

40328

Saleem Frank 45296

Nik M. Ahmadi

Eng. Kamal

811231

305, St. 25, D-4, Ph. 1, Hayatabad, Peshawar Hossaini

43693

$84-E$. Rahman Baba Rd, Abdara Rd, U/T, Pesh.

Abdul Salam

812530

25 B-2, Rehman Baba Rd, U/T, Peshawar

Dr. Zabihullah

41188

84-E Rahman Baba Kd, Abdara Rd, U/T, Pesh.

Eng. Salimee

45306

130, 1st Floor, Gul Haji Palza, J. Rd, Pesh.

DARA

Alfaroq St., Shaheen Town, J. Rd, Peshawar

10, Gulmohar Lane, U/T, UPO Box 855, Pesh.

DCA $108-\mathrm{E}, \mathrm{Block} 4, \mathrm{~S} / \mathrm{T}, \mathrm{PO}$ Box 36 , Quetta

DgCAR

DS 819 Jamrud Rd, PO BOX 792, Peshawar

Dr. Sanaulhaq

41188

M. Ali Targhi 43281

Ultich Larsen 44237

Ghulam Zekria 442515

Tino Hennecken40871

EAFA

ECAR

EDS

EMAR

ERU

ESAR

FRF

GAA

Street 9, Jamal Rd, Shaheen Town, Peshawar 58-C, University $\mathrm{rd}, \mathrm{U} / \mathrm{T}$, UPO BOX 800, Pesh. Manzal Bagh, Haji Abush House, Kandahar Afgh Street 2, Jamal Rd, Shaheen Town, Peshawar 37-D, Circular Lane, U/T, Peshawar 351, D-2, St 47, Ph 1, Hayatabad Peshawar 2, St. C 9/2, Zone 2, Jalalabad, Afghanistan Canal Rd, Academy Town, UPO Box 870, Pesh. 108-E S/T, PO Box 36, Quetta

GAF

GP-UK

St 13, Wazir Akbar Khan, Kabul, Afghanistan 17-B, Gulmóhar Lane,U/T, GPO 1230, Peshawar

A. Ghfoor Sana 42987

DR. Jawed Iqbal 41500

Wali Karzie 442968

DR. Mujahid 841454

Eng. Gh.Farooq 842793

Eng. A.Ghani 813935

Andren Graham $43912 / \mathrm{P}$

A.Manan Amiri 44608

Mohd Younus

4425155

R. Williamson $\mathrm{Nil}$

Dr. A. Ghafoor

42613 29, Street $32, F-6 / 3$, Islamabad

Randy Adams

217920 
GRC

GRSP

HAF

HAFO

HCI

HDCAW

HELP

HERI

HI

HRAA

HRCA

HRP

HT

HWRA

IAAAE

IAHC

IAM

IAU

ICD

ICRC

IFRCS

IHSAN

I I RO

IMC

IOC

IOM

IRC

ISRA

JAMS

JCE

JIFF

JWMM

KMA

$\mathrm{KNF}$

KPRO

KRCS

KWA

LB I

LDD I

LKRO

LQO

$\triangle \mathrm{RO}$

MADERA

MAI

MARUF

MAWA

MCI

MCPA

MDC

MDM

MERLIN

MF

MHC

MMC

MPO
50-C Hazara Housing Society, Alam.Rd, Quetta 2, 3rd Floor, Khatak plaza, U/T, Peshawar 10-B Park Lane, U/T, PO Box 819, Peshawar 24-C4, Park Avenue, U/T, UPO Box 1010, Pesh. 39-D S. J.Afghani Ad, PO Box 880, Peshawar 233, st 24, D-4, Ph 1, Hayatabad Peshawar 9, Railway Road, U/T, PO Box 912, Peshawar Kandahar Hotel, Kandahar Afghanistan Handicap Int, PAK/AFG, PO 477, Quetta 171-J Block 3, Satellite Town, Quetta 234, Block 2, Mini Market, S/T, Quetta 31, 3rd Floor, Spinzar Plaza, J. Rd, Pesh. Char Rahi Haji Yaqoub, Shahr Naw, Kabul, Afg. 243, Block 2, Mini Market, S/T, Quetta 243, Block 2, Mini Market, St, Hayatabad.Pesh. 90-E, Block 5, S/T, PO Box 293, Quetta Wazir Akbar Khan, Lane 1, St 15, Kabul, Afgh. Markza Al-Islami, Sardar Garai, Gt Rd, Pesh. 3-C, Gulmohar Lane, U/T, PO Box 813, Pesh. 40, S.J.Afghani Rd, U/T, Peshawar (IFRC), 43-D, S.J.Afghani Rd, U/T, Peshawar 2, Zeart Street, Board, J.rd, Peshawar 25, Park Lane, U/T Jamrud Rd, Peshawar 32-J/3,ST. 3, Ph. 2, Hayatabad, Peshawar $180,0-1, \mathrm{Ph}$. 1, Ghazali Rd-3, Hayatabad, Pesh. 25, St. 59, F7/4, Islamabad

80-3, Old Bara Rd, U/T, GPO Box 504, Pesh. 18-A, Park Rd, U/T, PO Box 887, Peshawar 3-C, Circular Rd, U/T, UPO Box 847, Pesh. 208 Gul Haji Plaza, J. Rd, Peshawar 1, Rahman Baba Rd, U/T, Peshawar c/O IAM, Wazir Akbar Khan Kabul AFghanistan 97, Block 1, S/T, Near Gole Masjed, Quetta 1,2 nd F1, Khayber View Plaza, Peshawar Tangee Charu, Dehsabz, PO 3170, Kabul, Afgh. Abdara Chowk, J. Rd, PO BOX 782, Peshawar C/O 310 Gul Haji Plaza, J. Rd, Peshawar $3304 / 5$ Safaid Dheri, UPO Box 1055, Peshawar 1, Arbab Rd, U/T, P.O.BOX 906, Peshawar 49, Block 1, Gol Masjid, S/T, Quetta 10, St. $52, F-7 / 4$, Islamabad

2 , Khyber St., Academy Town, U/T, Peshawar 53 C-II, Gulmohar Lane, U/T, Peshawar

303-C, Gul Haji Plaza, J. Rd, Peshawar St. 11, Shaheen Town, U/T, Peshawar
391, D-4, St. 27, Phase 1, Hayatabad, Pesh. 10, Arbab Karam Khan Rd, Box 314, Quetta 13, St. 9, F-8/2, Islamabad

\section{Jamal Food Industry Mumriz, Peshawar}

Lani 5, st 15, Wazir Akbar Khan, Kabul, Afgh. 9-10/449B, Kilishabo Rd, Chaman H, Quetta

Tajabad Boad, Jamrud Rd, Peshawar

Mission Hospital Compound Dabgari, Peshawar 310, GUL Haji Plaza, Jamrud Rd, Peshawar 5, street 4, Nasir Bagh Rd, Board, Peshawar
Zaman Atrafi 830774

Esehaq Zeerak 812530

Van Der Bunt 840621

Eng. S. Jawed 44677

A. S. Khadar 840524

Miss. Qudsia 810970

Ortwin Joch 840776

Shah Hossain Nil

Alain Robyns 830427

H. Haqyar

442922

Eng.Shah Mohd 443838

G. A. Merzai 842379

Dr. Farid

F. Akhondzada

G.H.Mutawazi

Baqi Haqani

Bruce Gibbs

M. Ali Shah

DE Colombani

Eric Schnider

Bob Mckerrow

Eeg.Rrz Mohd

Abu Abduhu

DR. Anwarulhaq

Dr. Abdullah

D. Alvero

32934

443838

811441

444780

25723

215954

41496

41371

843116

842954

840272

Linda Anderson41274

E.A. Rahim 42245

Shawali Zarif 44350

Dr, H.Mujadidi 44483

Dr. M. Kabir 41278

Beryl Beynon 25723

Haji M. Ali 441209

Mohd. Daud

D. Schettion $\mathrm{Nil}$

A. Elsanousi 840521

S. Jamal Nanir 44682

Mohd Mustafa 840249

Muzanil Mohd 42595

Abdul Aziz

447989

819908

Kazem Niazi 44278

J. F. Cautain 840546

Salim Emad 43203

A. H. Lat ify

S.A. Tahseen

Thomas Brown

Eeg. Sayed Aqa

Shohib Hakimi

Dr. Chazawn

oliver

Abu Osman

Dr. Rabiha

Dr. Reha

843618

810391

442863

855939

229236

081470

828856

842683

211289

44682

842833 


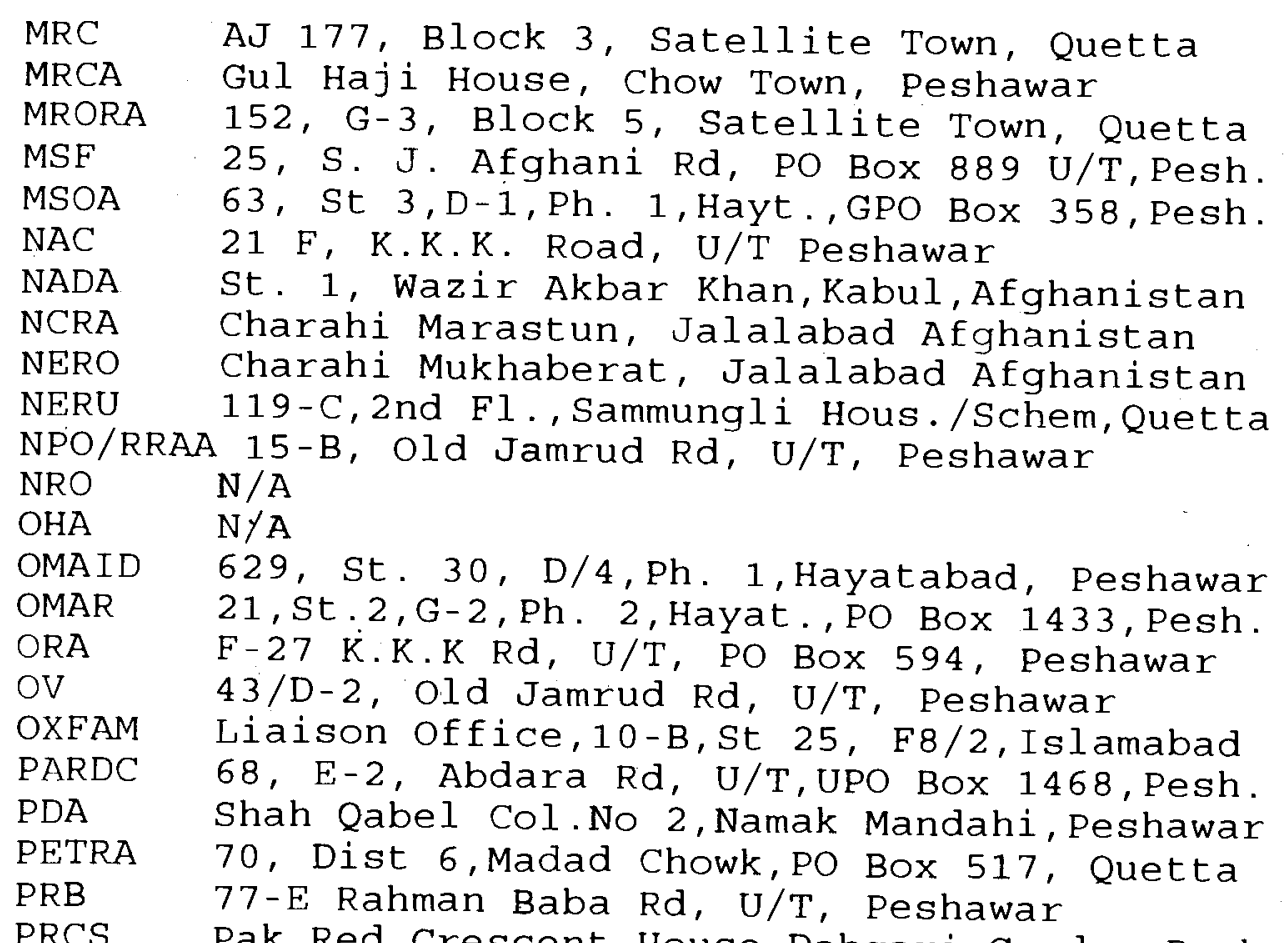
Pak. Red Crescent House, Dabgari Garden Pesh.M.A. Shah

PRS

PSD

PSF

RADA

RAFA

RAH

RBS

RDA

RDM

RDP

RDW

ROAOW

RPA

RPSA

RSSA

SAA

SADA

SAMSOR

SAVE

SC-US

SCA

SCF - UK

SERVE

SGAA

SGRO

SHACA

SJAWO

SNI

SO

SOLID SOS-PG SRA 1 , St. 2, Kababyan, Abshar Colony, Peshawar B-85, Gulistan Town, P.O Box 411, Quetta St 15, Wazir Akbar Khan, Kabul Afghanistan 1, Jamal Rd, Shaheen Town, GPO Box 356, Pesh. $43 / \mathrm{Block} E, \mathrm{~S}$. J. Afghani Rd, U/T, Peshawar 280 , L/2, Phase 3, Hayatabad Peshawar

228, Gul Haji Plaza, UPO Box 1424, Peshawar 1 -B Afridi House, Abdara Lane, U/T, Peshawar Murad Plaza, J. Rd, GPO Box 615, Peshawar 766-T, St.3, Jamal Rd, Shaheen Town, Pesh. Canal Rd, U/T, Peshawar

$$
\begin{aligned}
& \text { St. } 9 \text {, Jamal Rd, Shaheen T., GPO Box 356, Pesh. } \\
& \text { N/A }
\end{aligned}
$$

$\mathrm{N} / \mathrm{A}$ Sanzair Ada, Shahidan Chowk, Kandahar Afgh. $\mathrm{N} / \mathrm{A}$

\section{1 , St 13, E-3 Hayatabad Peshawar} 23, College Rd, F-7/2, PO Box 1952, Islamabad 24-D/E, Chinar Rd, U/T, UPO Box 689, Pesh. 194-A, F-7/3, College Rd, Islamabad 5 Mulbery Rd, GPO Box 477, U/'T, Peshawar ITC, Phase 5, Hayatabad, UPO Box 969, Pesh. Sarobi Rd, Jalalabad City, Afghanistan Flat 4, 1st Floor, Khattak Plaza, Peshawar Darul Khair, Nemat Mahal, PO Box 974, Pesh. 60-E, Canal Rd, U/T, GPO Box 354, Peshawar Hujra House, Old Bara Rd, U/T, Peshawar ITC, Phase 5, Hayatabad, UPO BOX 799, Pesh. $448-\mathrm{v}, \mathrm{Block} 3, \mathrm{~S} / \mathrm{T}$, Quetta
6, 2nd Fl, Khayber Pla, Abdara Chawk, Pesh. 77. Muradabad, Board, Peshawar Shuhada Clinic, Alamdar Rd, Naserabad, Quetta
Bashir Ahmad Ahmad Zafar N.K. Barakzai Ton Koene Fazel Menhaj Ms. Eva Sovre S. M. Afzali Sayed Ghufran Mohd Akran Eeg.Jan Mohd M. Aman Joya Mir Ahmad Shirzad

Qazim Wufa

F.Karim Fazel T.Grosshauser Mahboobullah Sue Emmott Shah Mohammad Munawar Khan Prof. Akbarzad Eng. M. Kabir

440683

843926

448190

42400

813512

41346

843618

2667

$\mathrm{Nil}$

880027

41129

440543

44252

810277

812919

841280

40410

261883

842943

211120

79894

41641

210836

S.A.S.Gallani 274179

M.H.Abdullahi 838945

G. Bernard 19873

Dr. Baluch 43358

Eng. Rashid 40893

Eeg. Khalil Nil

Jorgen Persson44784

Zaid Haidary 41092

A. A. Maihamyar 41940

N. Yousufi 42825

A. Wahab Kamal 42947

Belqis Baluch 45458

M. Nader Azimi 442304

Sayed Abbas 842617

Batensha Safi 842187

A. Wali Wafa 440683

M. Raoof 921799

A.Wajid Adil 810287

G.K. Helseth 214197

Carl E.Wiberg 44286

Michael Scott 218214

Steve Graig 41706

Anthony Smith 821256-7

-..- $\mathrm{Nil}$

Dr. A. Hadi 40734

H.G. Dastagir 42763

Georg Taubmann41130

Dr.Sima Samar 830318

Raoul De Torcy840228

Amir M.Ahmadi 812456-7

Mohd Barrch 447852 
SRCDA 207-L, Block 3, Satellinte Town, Quetta M. Naim Amin SRCS 2, Gulmohar Rd, U/T, Po Box 397, Peshawar M. Al-Dhaif SRDA 2nd Building, St 2,S.J. Square, Jalal, Afgh.Dr. MuKhlis SRO 2nd Building, St 2, S. J. Square, Jalal, Afgh.
$448-\mathrm{V}$, Block 3, Satellite Town Quetta

SRRC Mohd Barich

START 51-C, Park Avenue, U/T, Peshawar SWAARR 286, Block 2, S/T, PO Box 227, Quetta G.G. Sajedi

441316 840213 2016 447852 821686 SWARO

TAMIR

TDH

\section{T'RO}

UAAR UAE-RCS UMCA UNO/EPA VARA VSO WFA WRA WRC WRF WROR WUFA 243, Mini Market, Satellite Town, Quetta Eeg. M. Shah 41081 A.Wali Hamidi 440579 Dr. S. Shahab 444341 Khalil/T, Near Railway Tarck Board, Peshawar c/O ACBAR Office, Kabul Afghanistan

Dr. I Sumadi Cindy Dubble Angor Bagh, City Distric IV, Jalalabad Afgh. Shapur Khan 2 , S. J. Afghani Rd, U/T Peshawar Nasiri

- - - 54006 Block 5, Satellite Town, Quetta 2, Khyber Street, Gulabad, J. Rd, Peshawar

Babakarkhel Prof.A.Azimi Najmuddin $\mathrm{Nil}$ $\mathrm{Nil}$ 44375 $\mathrm{Nil}$ 41697 44536 56-C, Old Bara Rd, U/T, GPO Box 967, Pesh. 414-U, Block 3, Satellite Town, Quetta

Afaq Ali 444897 17, St. 50, F-7/4, PO Box 2543, Islamabad M. Enam Wak 815574 414A, Gul Haji Plaza, PO Box 1103, Peshawar $\mathrm{N} / \mathrm{A}$ 38-D, Park Rd, U/T, Po Box 1097, Peshawar Karte Aryana, Nahya 2, Mazari Sharif, Afgh. A.J. Siddiqi 44300 766-T, St. 3, Jamal Rd, Shaheen T. Peshawar YARA $78-\mathrm{E}$, Rehman Baba Rd, U/T, Peshawar Sarwar Nikzad Nil ENG. A. Shah 42825 Rasul Amin 840318 Dad M. Khalid 841749 


\section{AMENDMENTS TO THE 8TH EDITION OF THE DATABASE}

Afghanistan Nothilfe e. V. (ANH) has changed its name to Afghan/German help Coordination Office (AGHCO).

Action Internationale Contre La Faim (AICF) has changed its name to Action Contre La Faim (ACF)

The abbreviation (TAMER), in the index as well as on page 253 of volume III, should read (TAMIR).

HCR/NOVB stands for UNOCHA/NOVIB, OMAR's funding source, on pages 174 and 175 of the third volume. HCR is mistakenly used instead of UNOC which is a short abbreviation for UNOCHA. 